\title{
Development of Wearable Ultrasonic Sensors for Monitoring Muscle Contraction
}

\author{
By \\ Ibrahim AlMohimeed
}

A thesis submitted to the Faculty of Graduate and Postdoctoral Affairs in partial fulfillment of the requirements for the degree of

Master of Applied Science in Biomedical Engineering

Ottawa-Carleton Institute for Biomedical Engineering

Department of Systems and Computer Engineering

Carleton University

Ottawa, Ontario, Canada

August 2013

Copyright $(9$ 2013, Ibrahim AlMohimeed 
The undersigned recommend to

Faculty of Graduate and Postdoctoral Affairs

acceptance of the thesis

\title{
Development of Wearable Ultrasonic Sensors for Monitoring Muscle Contraction
}

\author{
Submitted by Ibrahim AlMohimeed \\ in partial fulfillment of the requirements for the degree of \\ Master of Applied Science in Biomedical Engineering
}

Yuu Ono

Thesis Supervisor

Roshdy H.M. Hafez

Chair, Department of System and Computer Engineering

Carleton University 


\section{Abstract}

This thesis presents the development of a wearable ultrasonic sensor to monitor muscle contractions. A flexible and lightweight ultrasonic sensor was constructed using a polyvinylidene fluoride piezoelectric polymer film, sandwiched by electrodes on the top and the bottom, and sealed by protection/insulation layer. A numerical simulation model of the sensor, based on Mason's electric equivalent circuit model of piezoelectric resonators, was developed. The internal losses of piezoelectric polymers were considered in the mathematical representation of the numerical simulation model for accurate prediction of its ultrasonic performance. The performance and frequency characteristics of the developed sensors were investigated by numerical simulations and experiments. The results of the numerical simulations and experiments show that the developed sensor operates in dual frequencies due to the effect of the non-piezoelectric layers, specifically

of the silicone adhesive layers. The ultrasonic signal strength of the sensor with respect to the sensor size was investigated.

Experiments were conducted to demonstrate the capability of the developed wearable sensor for monitoring static and dynamic muscle contractions. The muscle thickness changes measurement has been used to monitor muscle activities during contractions. The flexibility and lightweight of the sensor allows the sensor to be attached to the body area of interest without restricting the underlying tissue movements, which is not feasible using a conventional handheld ultrasonic probe. 


\section{Acknowledgements}

I would like to express the deepest appreciation to my supervisor Dr. Yuu Ono, Associate Professor of Systems and Computer Engineering, Carleton University for his time, expertise, guidance, encouragement, and support.

I would also like to acknowledge Carleton University for the opportunity, and Majmaah University represented by Saudi Arabian Cultural Bureau (SACB) for the scholarship and the financial support.

Many thanks to my colleagues Zhen Qu, Hisham Turkistani, and Andy Huang for their help and time throughout my research work. They were always willing to share their knowledge and experiences.

I would also like to extend my thanks and appreciation to Nagui Mikhail, Department of Electronics, for his assistance and lab equipment.

Lastly, I would like to express my heart-felt gratitude to my father, Rasheed, my mother, Norah, my siblings, and my friends for their encouragement, prayers, and persistent support throughout my life. Besides, there are no words can express my love

and appreciation to my wife, Manal, for all the precious support, patience, and words of encouragement. 


\section{Table of Contents}

Abstract

Acknowledgements $\quad$ iv

Table of Contents $\quad$ v

List of Figures $\quad$ ix

List of Tables $\quad$ xiv

List of Abbreviation $\quad$ xv

List of Symbols $\quad$ xvi

Chapter 1: Introduction 1

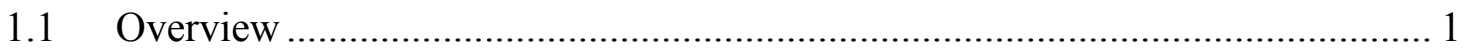

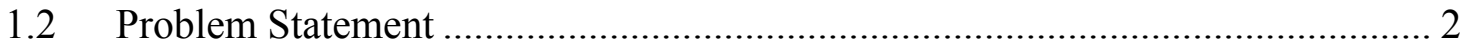

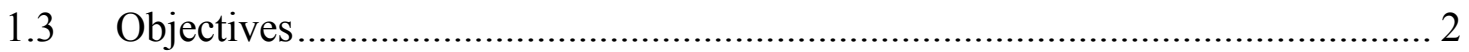

1.4 Thesis Contribution .................................................................................. 3

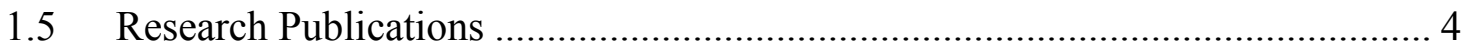

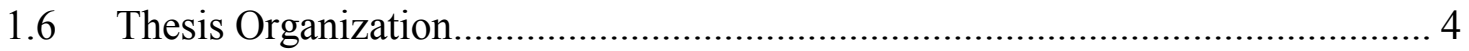

Chapter 2: Background and Technical Review 6

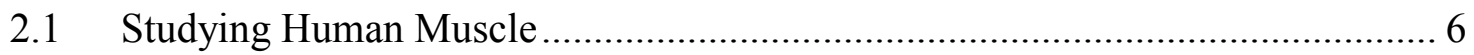

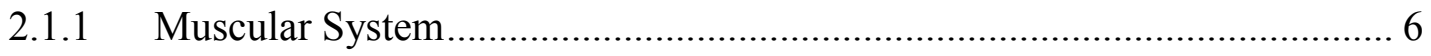

2.1.2 Skeletal Muscle Contraction ................................................................. 7 
2.1.3 Skeletal Muscle Monitoring................................................................ 8



2.2.1 Piezoelectric Effect .............................................................................. 11



2.2.3 Portable Ultrasonic System................................................................ 15

2.2.4 Ultrasound Physics......................................................................... 22

2.3 Ultrasound Measurement System ………….............................................. 26

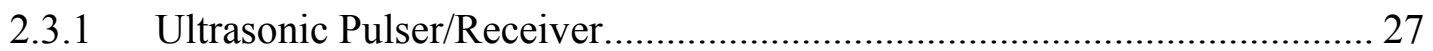

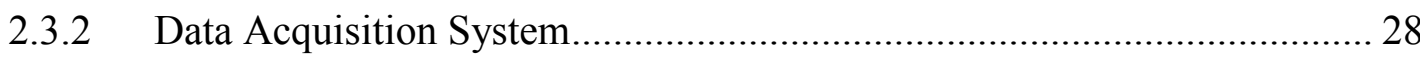

Chapter 3: Sensor Development 30

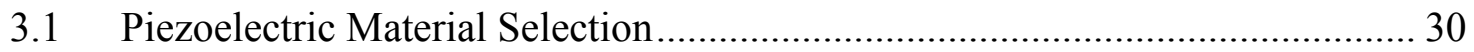

3.2 Developed Sensor Construction .................................................................... 33

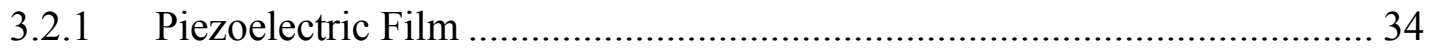



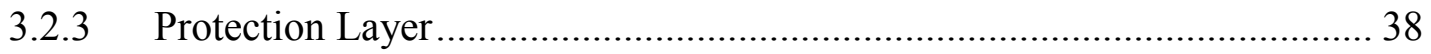

Chapter 4: Numerical Simulation Model $\quad 40$

4.1 Mathematical Expression of the Sensor ......................................................... 40

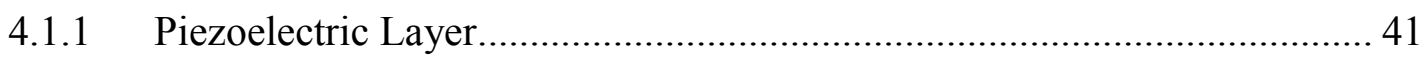

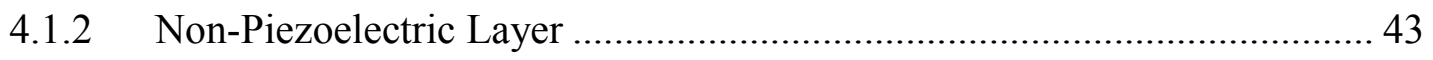

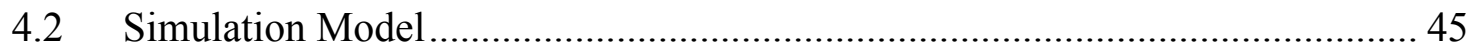




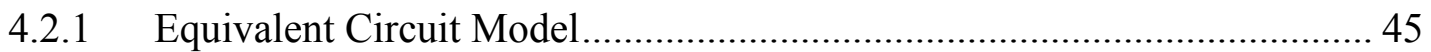

4.2.2 Sensor Impedance Derivation ................................................................. 48

4.2.3 Conversion Efficiency ........................................................................... 52

Chapter 5: Ultrasonic Sensor Characterization 53

5.1 Numerical Calculation of Sensor Characteristics............................................. 53

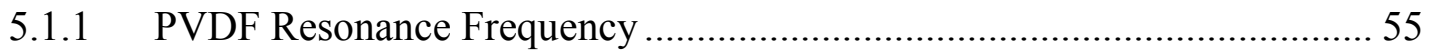



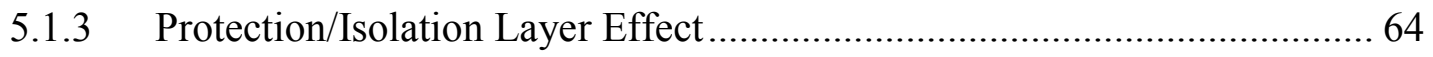

5.2 Experimental Investigation of Sensor Characteristics ……………………....... 74

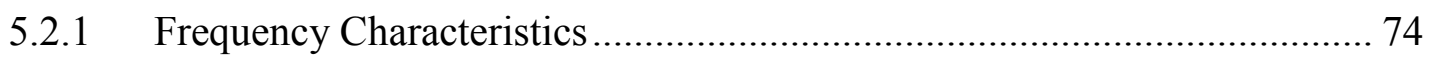

5.2.2 Ultrasonic Signal Strength ......................................................................... 78

5.2.3 Backing Material to Improve Frequency Characteristics .......................... 82

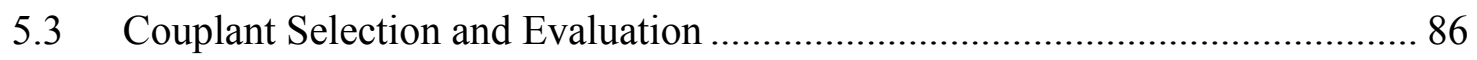

$\begin{array}{lr}\text { Chapter 6: Muscle Monitoring } & 89\end{array}$

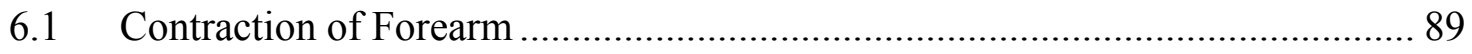

6.1.1 Pulse-Echo Technique …………………….......................................... 89

6.1.2 Through-Transmission Technique ............................................................. 96

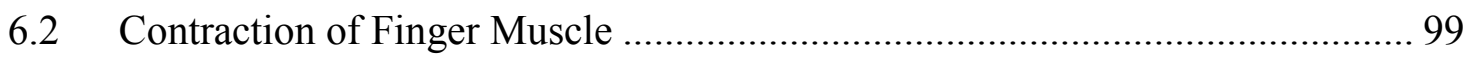

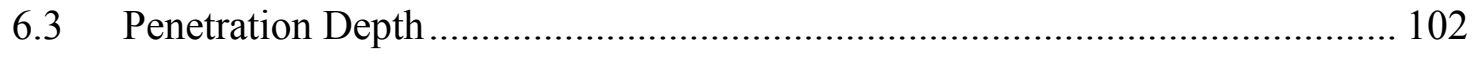

$\begin{array}{ll}\text { Chapter 7: Conclusions and Future Works } & 104\end{array}$ 


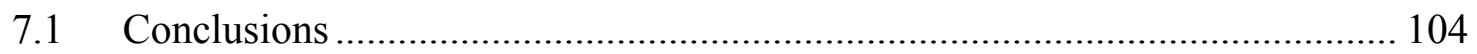

7.2 Future Works................................................................................... 105

$\begin{array}{ll}\text { References } & 107\end{array}$ 


\section{List of Figures}

Figure 2.1: The principles of ultrasonic measurements. (a) pulse-echo and (b) throughtransmission techniques. 10

Figure 2.2: Piezoelectric thickness-extensional mode oscillation. 12

Figure 2.3: Schematic of basic ultrasonic probe with single transducer element. Based on [23], [28] 13

Figure 2.4: (a) ADR 2130, (b) Toshiba SAL-20A [31]..... 16

Figure 2.5: (a) SonoSite NanoMaxx [32], (b) GE Vscan [33], (c) Siemens ACUSON P10 [34], (d) Current Solutions US Pro 2000 [35], (e) Interson Corporation SeeMore Ultrasound Imaging USB probes [36]. 17

Figure 2.6: (a) Pocket-size ultrasonic bladder volume sensor [43], (b) wearable ultrasonic bladder volume [44], (c) wearable ultrasound for cardiac monitor [39], (d) wearable ultrasound probe [45], (e) wearable Doppler ultrasound [41], (f) ultrasonic surface

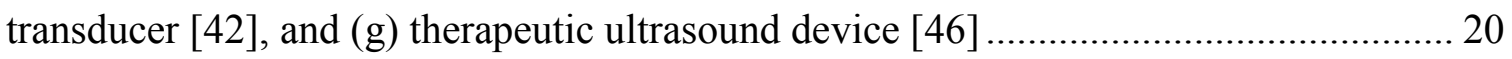

Figure 2.7: Ultrasound System Connection for the pulse-echo technique...................... 26

Figure 2.8: Ultrasound System Connection for the through-transmission technique....... 27

Figure 2.9 : Ultrasonic pulser/receiver devices: (a) NDT 5900PR, (b) DPR 300........... 28

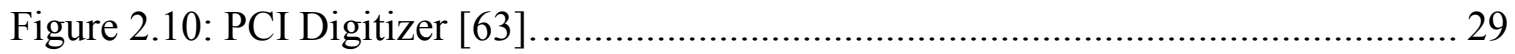

Figure 3.1: schematic representation of the ultrasonic sensor design ............................ 33

Figure 3.2: photographs of the ultrasonic film sensor developed $(1.5 \mathrm{~cm} \times 1.5 \mathrm{~cm}$ active

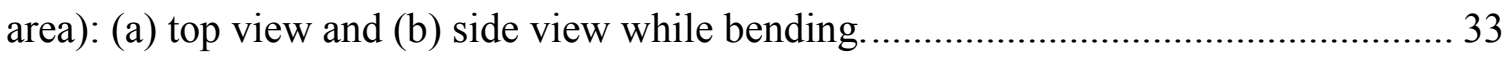

Figure 3.3: Metallized PVDF film sheet (silver paint electrode)................................. 35 
Figure 3.4: PVDF resonance frequency with respect to PVDF thickness. 35

Figure 3.5: PVDF film sandwiched by top and bottom electrodes. 37

Figure 3.6: The active and the interconnection area of the developed sensor. 38

Figure 4.1: Three ports network of piezoelectric vibrates in thickness mode. 43

Figure 4.2: General layers configuration of an ultrasonic sensor. 44

Figure 4.3: Mason's equivalent circuit. 46

Figure 4.4: Developed sensor basic layers structure. 46

Figure 4.5: The equivalent circuit model of the developed sensor. 47

Figure 4.6: Developed sensor's equivalent circuit of the back side (a), and the equivalent back layers impedance, $\mathrm{Z}_{\mathrm{BL}},(\mathrm{b})$. 48

Figure 5.1: Frequency response of ultrasonic sensor composed of PVDF film only; (a) sensor structure, (b) transducer loss (TL). 56

Figure 5.2: Frequency response of ultrasonic sensor composed of PVDF film only; (a) impedance magnitude, (b) impedance phase. 57

Figure 5.3: Frequency response of ultrasonic sensor showing the effect of the internal losses; (a) transducer loss, (b) conversion loss, (c) matching loss. 59

Figure 5.4: Numerical calculation results of the fundamental resonant frequency for the PVDF film as a function of front and back silver paint layer thickness; (a) sensor structure, (b) fundamental resonant frequency.

Figure 5.5: comparing the effect of silver paint and copper-nickel (top and bottom electrodes) to the PVDF resonance frequency; (a) matching loss (ML), (b) conversion loss (CL), (c) transducer loss (TL) 63 
Figure 5.6: transducer loss of ultrasonic sensor composed of $110-\mu \mathrm{m}$ thick PVDF film with front and back silicone adhesive; (a) sensor structure, (b) transducer loss (TL)..... 65

Figure 5.7: Calculated loss parameters of ultrasonic sensor composed of $110-\mu \mathrm{m}$ thick PVDF film and 25- $\mu \mathrm{m}$ thick front and back silicone adhesive; (a) sensor structure, (b)

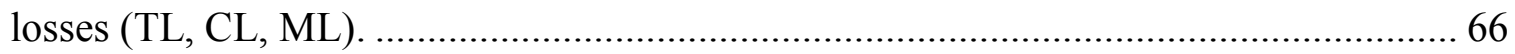

Figure 5.8: The transducer loss of ultrasonic sensor composed of $110-\mu \mathrm{m}$ thick PVDF film with Polyimide layers in both sides; (a) sensor structure, (b) transducer loss (TL).. 67 Figure 5.9: The loss parameters of ultrasonic sensor composed of $110-\mu \mathrm{m}$ thick PVDF film with front and back 13- $\mu$ m thick Polyimide layers; (a) sensor structure, (b) losses. 67 Figure 5.10: Structures of the three PVDF sensors compost; (sp) silicone adhesive and polyimide film layers, (s) silicone adhesive layers, (p) polyimide film layers (p). 69 Figure 5.11: Calculated loss parameters of ultrasonic sensor composed of structures "sp", "s" and "p" ; (a) conversion loss (CL),(b) matching loss (ML), (c) transducer loss (TL).70 Figure 5.12: Structures of the three PVDF sensors composed of; (esp) electrode and protection layers, (sp) silicone adhesive and polyimide layers, (e) electrodes layers. ..... 72 Figure 5.13: Calculated loss parameters of ultrasonic sensor composed of structures “esp", "sp" and "e" ; (a) conversion loss (CL), (b) matching loss (ML), (c) transducer loss (TL). 73

Figure 5.14: Photo of sensor glued to a Plexiglas tube ........................................... 75

Figure 5.15: Sketch of the experiment procedure...................................................... 76

Figure 5.16: Frequency spectrum of the reflected ultrasonic pulse signal acquired by the developed sensor at several depths from the reflector; (a) $4 \mathrm{~mm}$, (b) $12 \mathrm{~mm}$, (c) $24 \mathrm{~mm}$ and (d) $36 \mathrm{~mm}$. 77 
Figure 5.17: Photo of ultrasonic sensor having different active areas size.

Figure 5.18: Schematic of experiment configuration for evaluation of ultrasonic signal

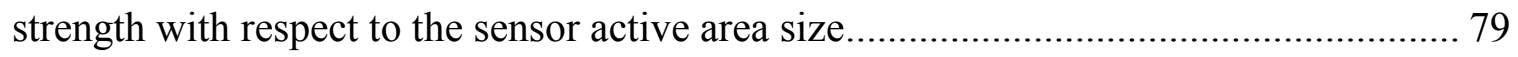

Figure 5.19: Ultrasonic signal strength comparison between various sensor sizes. ......... 80 Figure 5.20: Matching loss for several sensor sizes at the resonant frequency $8 \mathrm{MHz}, \mathrm{Z}$ is

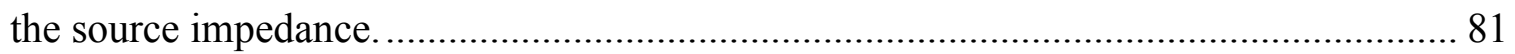
Figure 5.21: Photo of the ultrasonic film sensor developed with the backing material.... 82 Figure 5.22: Calculated loss parameters of ultrasonic sensor composed of $110-\mu \mathrm{m}$ thick PVDF film backed with silicone; (a) sensor structure, (b) losses (TL+CL+ML)........... 84 Figure 5.23: Calculated Transducer loss of are backing sensor and silicone backing sensor. 84 Figure 5.24: Frequency spectrum of the reflected signal pulse acquired with the sensor having a silicone backing. 85 Figure 5.25: Measured echo signal amplitude with the four different couplants: ultrasonic gel, honey, paper glue, and double-sided Polyimide tape. 87 Figure 5.26: The reflected ultrasonic signal amplitude variation over 24 hours for the ultrasonic gel, paper glue, and double-sided polyimide tape. 88 Figure 6.1: Wearable ultrasonic sensor attached onto forearm to monitor isotonic muscle contraction using pulse-echo technique, (a) at relaxed and (b) at contracted state. 91 Figure 6.2: Ultrasonic single reflected from the tissue-bone interface at the forearm. .... 91 Figure 6.3: Thickness variation during forearm muscle contraction monitored by the developed sensor. 92 
Figure 6.4: M-mode image of ultrasound measurement of forearm tissues by pulse-echo technique. 92

Figure 6.5: The setup of the experiment with the clinical ultrasound imaging system, (a) at relaxed state, (b) at contracted state. 94

Figure 6.6: B-mode images of forearm with the clinical ultrasound imaging system. The target muscle of interest and bone is shown in red, (a) at relaxed state, (b) at contracted state. 95

Figure 6.7: Wearable ultrasonic sensor attached onto forearm to monitor isotonic muscle contraction by through-transmission technique. 97

Figure 6.8: The received ultrasonic signal passed through the forearm tissue by transmission-through technique. 98

Figure 6.9: Thickness variation during forearm muscle contraction monitored by transmission-through technique. 98

Figure 6.10: M-mode image of ultrasound measurement of forearm tissues by transmission-through technique. 99

Figure 6.11: Wearable ultrasonic sensor attached into finger to monitor isometric muscle contraction. 100

Figure 6.12: Ultrasonic signal reflected from the tissue-bone interface at the index finger. 101

Figure 6.13: Thickness variation during finger muscle isometric contraction. 101

Figure 6.14: M-mode image of ultrasound measurement at finger. 102

Figure 6.15: Ultrasonic signal reflected from muscle-tibia bone interface at leg. 103 


\section{List of Tables}

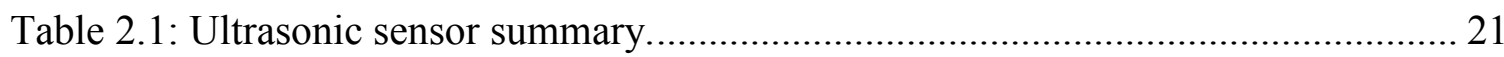

Table 3.1: Electromechanical properties of some piezoelectric materials [6] ................ 32

Table 5.1: Acoustic properties of the materials used in the numerical calculations......... 54 


\section{List of Abbreviation}

$\begin{array}{ll}\text { CL } & \text { Conversion Loss } \\ \text { DAQ } & \text { Data Acquisition } \\ \text { EMG } & \text { Electromyography } \\ \text { FDA } & \text { Food and Drug Administration } \\ \text { MI } & \text { Mechanical Index } \\ \text { ML } & \text { Matching Loss } \\ \text { MMG } & \text { Mechanomyography } \\ \text { MRI } & \text { Magnetic Resonance Imaging } \\ \text { P(VDF-TrFE) } & \text { Polyvinylidene Fluoride Trifluoroethylene } \\ \text { PVDF } & \text { Polyvinylidene Fluoride } \\ \text { PZT } & \text { Lead Zirconate Titanate } \\ \text { TE } & \text { Thickness Extensional Mode } \\ \text { TI } & \text { Thermal Index } \\ \text { TL } & \text { Transducer Loss }\end{array}$




\section{List of Symbols}

\begin{tabular}{|c|c|c|}
\hline Symbols & Definition & Units \\
\hline$A R C$ & amplitude reflection coefficient & \\
\hline$A T C$ & amplitude transmission coefficient & \\
\hline C & sensor capacitance & $\mathrm{F}$ \\
\hline$c^{*}$ & elastic stiffness & $\mathrm{N} / \mathrm{m}^{2}$ \\
\hline$c^{D}$ & piezoelectric elastic stiffness & $\mathrm{N} / \mathrm{m}^{2}$ \\
\hline$\varepsilon_{*}$ & dielectric permittivity & \\
\hline$\varepsilon_{o}$ & dielectric constant in vacuum & $\mathrm{F} / \mathrm{m}$ \\
\hline$\varepsilon_{S}$ & piezoelectric dielectric constant & $\mathrm{F} / \mathrm{m}$ \\
\hline$e$ & piezoelectric constant & $\mathrm{C} / \mathrm{m}^{2}$ \\
\hline$\gamma$ & propagation constant & \\
\hline$\lambda$ & wave length & $\mathrm{m}$ \\
\hline$v$ & longitudinal sound velocity & $\mathrm{m} / \mathrm{s}$ \\
\hline$\rho$ & density & $\mathrm{Kg} / \mathrm{m}^{3}$ \\
\hline$f_{o}$ & resonant frequency & $\mathrm{Hz}$ \\
\hline$\omega$ & angular frequency & $\mathrm{rad} / \mathrm{s}$ \\
\hline$h$ & transmitting constant & $\mathrm{V} / \mathrm{m}$ \\
\hline$k_{t}$ & coupling factor & \\
\hline$T$ & internal stress & $\mathrm{Pa}$ \\
\hline $\tan \delta_{s}$ & mechanical loss tangent & \\
\hline $\tan \delta_{m}$ & mechanical loss tangent & \\
\hline$z_{x}$ & axis along the film thickness & $\mathrm{m}$ \\
\hline$A$ & film area & $\mathrm{m}^{2}$ \\
\hline$d$ & layer thickness & $\mathrm{m}$ \\
\hline$d_{T}$ & tissue thickness & $\mathrm{m}$ \\
\hline$t_{u s}$ & ultrasound time delay & $\mathrm{s}$ \\
\hline$p_{o}$ & maximum acoustic pressure & $\mathrm{Pa}$ \\
\hline
\end{tabular}




\begin{tabular}{|c|c|c|c|}
\hline$I_{A}$ & & time-averaged ultrasound intensity & $\mathrm{W} / \mathrm{m}^{2}$ \\
\hline$I_{S P P A}$ & & spatial peak pulse average ultrasound intensity & $\mathrm{W} / \mathrm{m}^{2}$ \\
\hline$I_{S P T A}$ & & spatial peak temporal average ultrasound intensity & $\mathrm{W} / \mathrm{m}^{2}$ \\
\hline$B$ & & acoustic impedance of an area & $\mathrm{kg} / \mathrm{s}$ \\
\hline$Z_{A}$ & & equivalent acoustic impedance of circuit model & \\
\hline$Z_{C}$ & & equivalent acoustic impedance of circuit model & \\
\hline$Z_{b}$ & & acoustic impedance of the back medium & $\mathrm{Kg} / \mathrm{m}^{2} \mathrm{~s}$ \\
\hline$L$ & & equivalent acoustic impedance of the back layers & $\mathrm{Kg} / \mathrm{m}^{2} \mathrm{~s}$ \\
\hline$Z_{f}$ & & acoustic impedance of the front medium & $\mathrm{Kg} / \mathrm{m}^{2} \mathrm{~s}$ \\
\hline$Z_{t}$ & & electrical impedance of the sensor & $\mathrm{ohm}$ \\
\hline$Z_{s}$ & & electrical impedance of electric source & ohm \\
\hline$P_{o}$ & & electric power from the electric source & $\mathrm{W}$ \\
\hline$P_{t}$ & & electric power transmitted into the sensor & $\mathrm{W}$ \\
\hline$P_{m f}$ & & forward acoustic power & $\mathrm{W}$ \\
\hline$P_{m b}$ & & backward acoustic power & $\mathrm{W}$ \\
\hline$I_{\text {current }}$ & & electric current & A \\
\hline $\mathrm{V}$ & & electrical voltage & $\mathrm{V}$ \\
\hline$U$ & & particle velocity & $\mathrm{m} / \mathrm{s}$ \\
\hline$F$ & & force & $\mathrm{N}$ \\
\hline & -subscript & & \\
\hline & $\mathrm{pz}$ & Piezoelectric layer & \\
\hline & $\mathrm{f}$ & Propagation (front) medium & \\
\hline & $\mathrm{b}$ & Backing medium & \\
\hline & $\mathrm{pf}$ & Front polyimide film layer & \\
\hline & $\mathrm{pb}$ & back polyimide film layer & \\
\hline & sf & Front silicone adhesive layer & \\
\hline & $\mathrm{sb}$ & Back silicone adhesive layer & \\
\hline & ef & Front electrode layer & \\
\hline & $\mathrm{eb}$ & Back electrode layer & \\
\hline & BL & Total backing layer & \\
\hline
\end{tabular}




\section{Chapter 1: Introduction}

The following chapter is an introduction to this thesis and will provide an overview of the thesis organization. In addition, the objectives and contributions made throughout the research work will be presented.

\subsection{Overview}

The properties and characteristics of human muscles, as well as their behavior, are of great interest to many researchers. The musculature of the human body is closely related to the overall health and well-being of an individual. Understanding the muscle contraction and measuring its movement is of ongoing interest to some medical practitioners.

Ultrasonic imaging equipment is capable of providing real-time dynamic images of internal tissue motion associated with physical and physiological activities. The use of ultrasound to monitor the skeletal muscle contraction can be an objective way of evaluating muscle performance. For instance, the muscle fatigue affects the rate of the thickness variation of muscle over time.

The motivation behind the research presented in this thesis is driven by the ongoing need to improve the accuracy and reliability of long term monitoring of muscle activities. More specifically, the solutions to the challenges and the potential errors present in the current ultrasound transducers. 


\subsection{Problem Statement}

One of the parameters indicative of muscle activities is the thickness variation of muscle; as a change in muscle thickness is associated with muscle contractions. The ultrasonic method of monitoring muscle contractions using a conventional ultrasonic probe may result in issues that cause false detection or an inaccurate measurement of the muscle internal displacement. One of the issues is the motion artifact produced by the inconsistent movement of handheld ultrasonic probe that is used on the body's surface during the measurement. Also, the probe weight exerts a force against the body's surface that may restrict or limit the underlying tissue motion. In addition, the measurement may be limited within clinics or laboratory environments due to the handheld ultrasonic probe and the bulky ultrasonic system. The research in this thesis aims to improve the accuracy of the measurement and overcome the outlined obstacles.

\subsection{Objectives}

The main goal of the thesis is to develop a wearable ultrasonic sensor for continuous monitoring of skeletal muscle contractions, and investigate its performance. In addition, we want to evaluate ultrasonic couplants for the developed sensor and conduct in vivo experiments to monitor muscle contractions.

To reach this goal, a flexible and lightweight ultrasonic sensor made of polymer piezoelectric material is constructed in order to eliminate its mass-loading effect on the movement of muscles and other tissue during monitoring. Then, a numerical calculation of the developed sensor and measurement configuration is developed to simulate the 
frequency characteristics and the performance of the sensor. Also, experiments are performed to study and investigate the piezoelectric activities and behavior of the developed sensor along with the numerical simulation. Finally, a monitoring of the muscle thickness variation in the human subject during contractions is conducted to demonstrate the capability of the developed sensor.

\subsection{Thesis Contribution}

The following are the main research contributions presented in this thesis. They will be explained and discussed thoroughly in the following chapters:

- Designed and constructed a flexible, lightweight ultrasonic sensor. The design involves discussion of the sensor components' properties and characteristics.

- Derived mathematical equations based on the sensor's electric equivalent circuit model for numerical simulation of the sensor characteristics.

- Evaluated the performance and behavior of the sensor. The evaluation involves numerical simulations and experimental validations with different configurations of the sensor structures.

- Investigated selected ultrasonic couplants for long term muscle monitoring.

- Performed measurements of muscle thickness on human subject during contraction with the developed sensor. 


\subsection{Research Publications}

- Ibrahim AlMohimeed and Yuu Ono "Wearable ultrasonic transducer for monitoring skeletal muscle contraction," in Proceeding 33rd Symposium on Ultrasonic Electronics, Chiba, Japan Nov. 13-15, 2012.

- Hisham Turkistani, Ibrahim AlMohimeed, and Yuu Ono "Continuous monitoring of muscle thickness changes during isometric contraction using a wearable ultrasonic sensor," in Conference of the Canadian Medical and Biological Engineering Society, Ottawa, Canada May. 21-24, 2013.

- Ibrahim AlMohimeed, Hisham Turkistani, and Yuu Ono "Development of Wearable and Flexible Ultrasonic Sensor for Skeletal Muscle Monitoring," in IEEE International Ultrasonics Symposium, Prague, Czech Republic Jul. 21-25, 2013.

\subsection{Thesis Organization}

Chapter 1 introduces the overall concepts and topics of the thesis including the main problems and objectives.

Chapter 2 provides background knowledge about the principles of ultrasound and human muscle, along with an overview of muscle monitoring.

Chapter 3 describes the structure of the developed sensor and the construction procedures. It also provides a discussion about piezoelectric material selection. 
Chapter 4 derives the mathematical expression of the piezoelectric activities of the developed sensor based on the electrical equivalent circuit model.

Chapter 5 provides a detailed study and discussion about the frequency characteristics and the performance of different configurations of the developed sensor structures based on the numerical simulation and the experimental results. In addition, it introduces and discusses the properties of selected ultrasonic couplants for the proposed application of muscle monitoring.

Chapter 6 demonstrates the capabilities of the developed sensor for measuring muscle thickness changes during static and dynamic contractions.

Chapter 7 concludes the major findings in this thesis, and discusses the improvements and suggestions for future work. 


\section{Chapter 2: Background and Technical Review}

This chapter introduces the basics about human muscle physiology and the physics and principles of the ultrasound technique for monitoring muscle contractions. The contents of this chapter are fundamental to this thesis.

\subsection{Studying Human Muscle}

Many researchers have studied skeletal muscle and collected information about muscle functions, muscle architecture [1], muscle pathology [2], muscle fatigue [2], [3], stiffness [1], [2] and muscle size [1]. These details about muscle characteristics and properties are valuable information for the diagnosis of trauma, malignancies, infection and the neuromuscular disorders of musculoskeletal system [4]. They can also be helpful in various applications such as rehabilitation, sports medicine, and prosthesis control.

\subsubsection{Muscular System}

The muscular system of the human body provides the body's movement and strength, maintains posture, and has a number of other purposes. It contains three distinct types of muscles: skeletal muscle, cardiac muscle and smooth muscle. Generally, cardiac and smooth muscles are categorized as involuntary muscles. Whereas, skeletal muscles are categorized as voluntary muscles that can be consciously controlled. The skeletal muscles account for nearly $40 \%$ of human body weight [5], [6]. They are responsible for 
the support and movement of the skeleton. Moreover, the skeletal muscle has a complex structure. The muscle is composed of groups of fascicles, and each of these fascicles contains bundles of muscle fibers. Each muscle fiber consists of many rod-like myofibrils which contain the contractile element of the muscle.

\subsubsection{Skeletal Muscle Contraction}

In order for the skeletal muscle to contract, the motor neuron of the sematic nervous system simulates muscle fibers by an electric impulse at the neuromuscular junctions. Thus, the stimulus generates an action potential across the muscle fiber membrane (sarcolemma), which leads to the sliding of myofilaments. This series of events from nerve impulse to contraction is called excitation-contraction coupling [6].

Skeletal muscle contractions can be sorted into two groups, isometric and isotonic, depending on whether or not the muscle changes its length during contraction. [7]. In an isometric contraction, the muscle tension (the force exerted by the muscle) extends at a constant length, therefore resulting in no movement. It is also referred to as a static contraction. However, the isotonic contraction is a dynamic contraction in which the muscle length changes and movement occurs. The isotonic contraction can be subdivided into two primary types: concentric and eccentric contractions. In both, the muscle length changes and the movement occurs. The muscle length shortens with concentric contractions whereas it stretches with eccentric contractions. An example of a concentric contraction is lifting a load from the ground. On the other hand, lowering the load to the ground is considered an eccentric contraction. 


\subsubsection{Skeletal Muscle Monitoring}

For such muscle research, it is important to develop methods to monitor and record muscle activities noninvasively, and to analyze these data in order to obtain useful information about muscle architecture and functions. There are a number of approaches that have been employed for the assessment of skeletal muscles. Electromyography (EMG) is one of the common approaches that provides the electrophysiological features during muscle activation invasively by inserting needle electrodes to acquire the electrical activities near the muscle of interest [8], [9], or noninvasively by measuring the average of the electrical activities of the muscle tissue at the skin surface using the surface electrodes [10], [11]. Monitoring the electrical activity of the muscle with the EMG technique can be used to determine the muscle force [12], fatigue [2] and action potential conduction velocity [13], [14].

Mechanomyography (MMG) is another approach that records the mechanical motion on the body surface caused by the muscle contractions [15]. MMG uses a wide variety of vibration transducers for motion detection such as piezoelectric contact sensors, accelerometers and microphones [16]. In general, the MMG method provides an external representation of the underlying tissue motion.

Magnetic resonance imaging (MRI) provides a noninvasive high resolution image of the muscle tissue structure. It is one of important imaging techniques to obtain data about muscle thickness, length and width [1], [17]. However, MRI is less commonly used due to its high cost and time consumption during the measurement, and the lack of portability. 
Similarly, ultrasound imaging and measurements are a promising method for the noninvasive measurement of muscle size [1], thickness [18], [19], fiber pennation angle [20], facial length and cross sectional area during static and dynamic contractions [21]. In this thesis research, the developed wearable ultrasonic sensor is used to monitor muscle contractions noninvasively by measuring the thickness of the muscle, where the changes in muscle thickness during contraction is associated with the force exerted by the muscle [1]. There are a number of different ultrasonic measurement techniques [22]. In this thesis however, we conducted muscle monitoring experiments using two techniques: pulse-echo and through-transmission. Both techniques use an ultrasonic pulsed system that generates the ultrasound in the form of a short pulse wave.

In the pulse-echo technique, a single sensor is used as a transmitter and receiver. It emits the ultrasonic pulse signal into an object of interest (eg. a human body as in this thesis) the soft tissue then receives the reflected ultrasonic signal (echo) from the interface at the soft tissue-bone as shown in Figure 2.1(a).

In the through-transmission technique, two sensors are used. One works as a transmitter and the other is a receiver. The two sensors are located opposite each other in the propagation direction of the ultrasonic signal as shown in Figure 2.1(b).

In both techniques, the change of the muscle thickness is measured by calculating the traveling time of the ultrasonic signal through the tissue. 


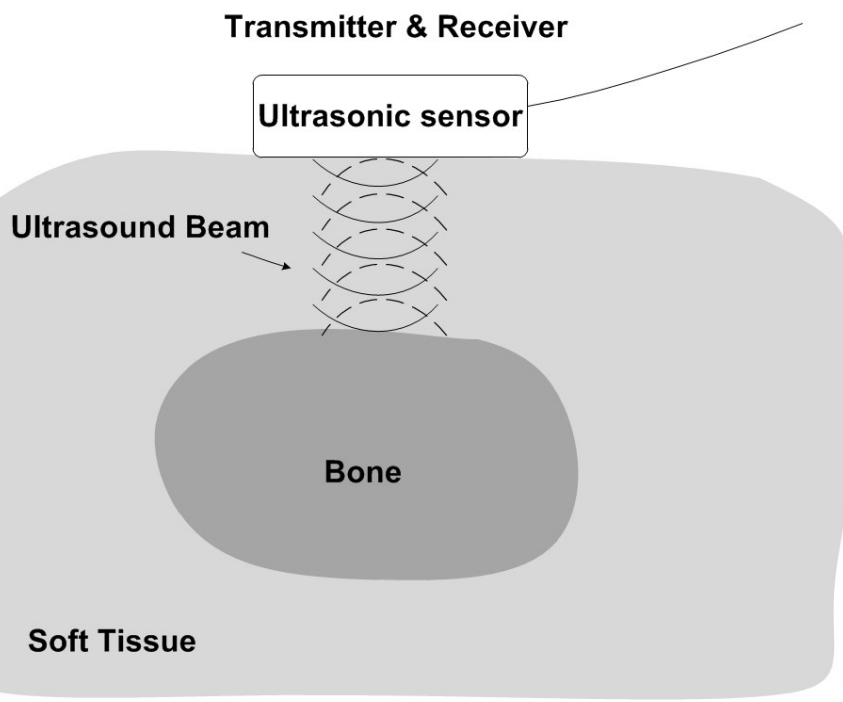

(a)

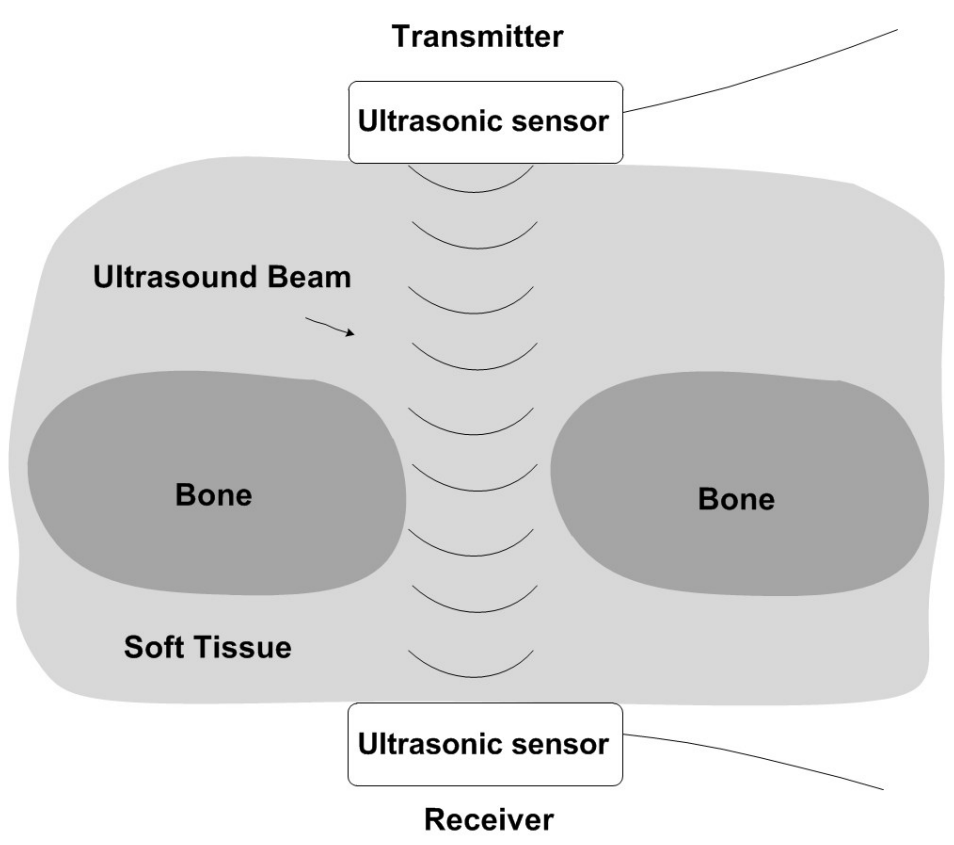

(b)

Figure 2.1: The principles of ultrasonic measurements. (a) pulse-echo and (b) throughtransmission techniques. 


\subsection{Ultrasound}

Ultrasound is a mechanical wave that propagates through a medium such as gas, liquid or solids, with frequencies above $20 \mathrm{kHz}$, exceeding the audible range of human hearing. The ultrasonic waves travel at a particular speed, depending on the medium they propagate through. The medical ultrasound typically uses a frequency range from $1 \mathrm{MHz}$ to $10 \mathrm{MHz}$ [23]. This range is a trade-off between spatial resolution which improves with frequency, and penetration depth which decreases with frequency [24]

Sound waves are divided into two basic types: longitudinal and transversal waves. For longitudinal waves, the particles motion of the medium is parallel to the direction of the wave propagation. Whereas the particles motion of the medium for transvers waves is perpendicular to the direction of the wave propagation [25]. Shear waves have a very high attenuation in the biological tissues so longitudinal waves are used in the medical ultrasound measurement.

\subsubsection{Piezoelectric Effect}

Ultrasound can be generated and received by an ultrasonic transducer made of a piezoelectric material. The piezoelectric effect is the ability of a material to produce an electrical charge in response to a change in its dimensions (deformation) [26]. When a pressure is applied on the surface of a piece of piezoelectric material, a voltage difference is generated across the opposite faces as shown in Figure 2.2. The one side has a positive charge and the other a negative charge. The inverse is also true. When a voltage is applied to the two opposite surfaces of the piezoelectric material, the piezoelectric material contracts or expands (become thinner or thicker). 
Thus, the piezoelectric material can be used as an ultrasound transmitter as well as receiver. It produces ultrasonic waves by applying an alternating voltage that causes the piezoelectric material to oscillate (expand and contract) at the same frequency as the voltage applied [26], [27].
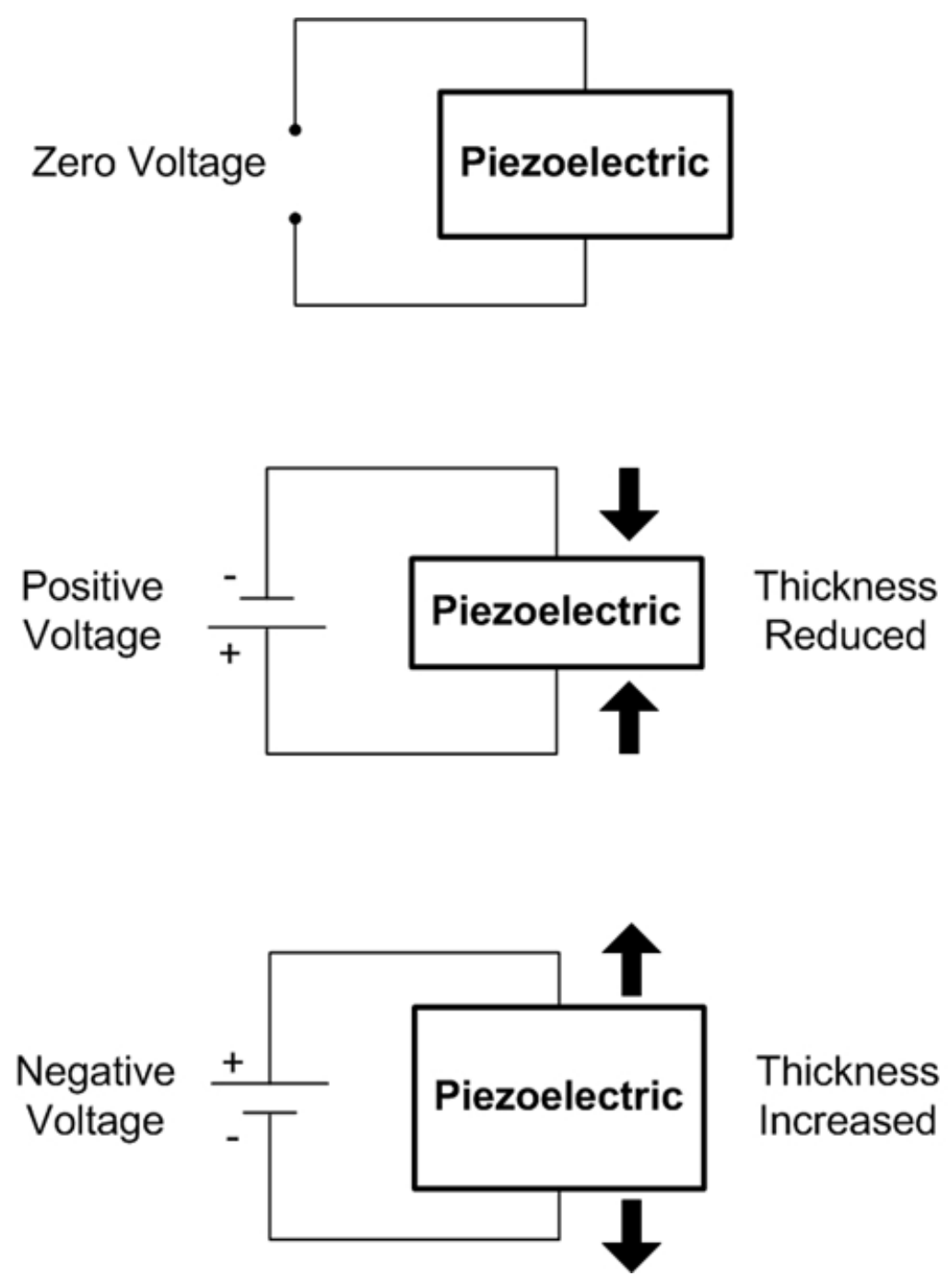

Figure 2.2: Piezoelectric thickness-extensional mode oscillation. 


\subsubsection{Ultrasonic Probe}

An ultrasonic transducer is a device that converts electrical pulses into ultrasonic pulses (transmitter), and conversely, converts ultrasonic waves into electrical signals (receiver) [28]. Modern medical ultrasound imaging systems use an ultrasonic probe that contains an array of transducer elements arranged in a number of different ways. Ultrasonic probes consist of the basic components: piezoelectric material, electrodes, matching layer(s), and backing material [25]. A schematic diagram of an ultrasonic probe with a single transducer element is shown in Figure 2.3. There may be an acoustic lens that extends across all the transducer elements for ultrasonic beam focusing if required. The piezoelectric material generally used for transducer in medical ultrasound systems is a ceramic material such as lead zirconate titanate (PZT) [28].

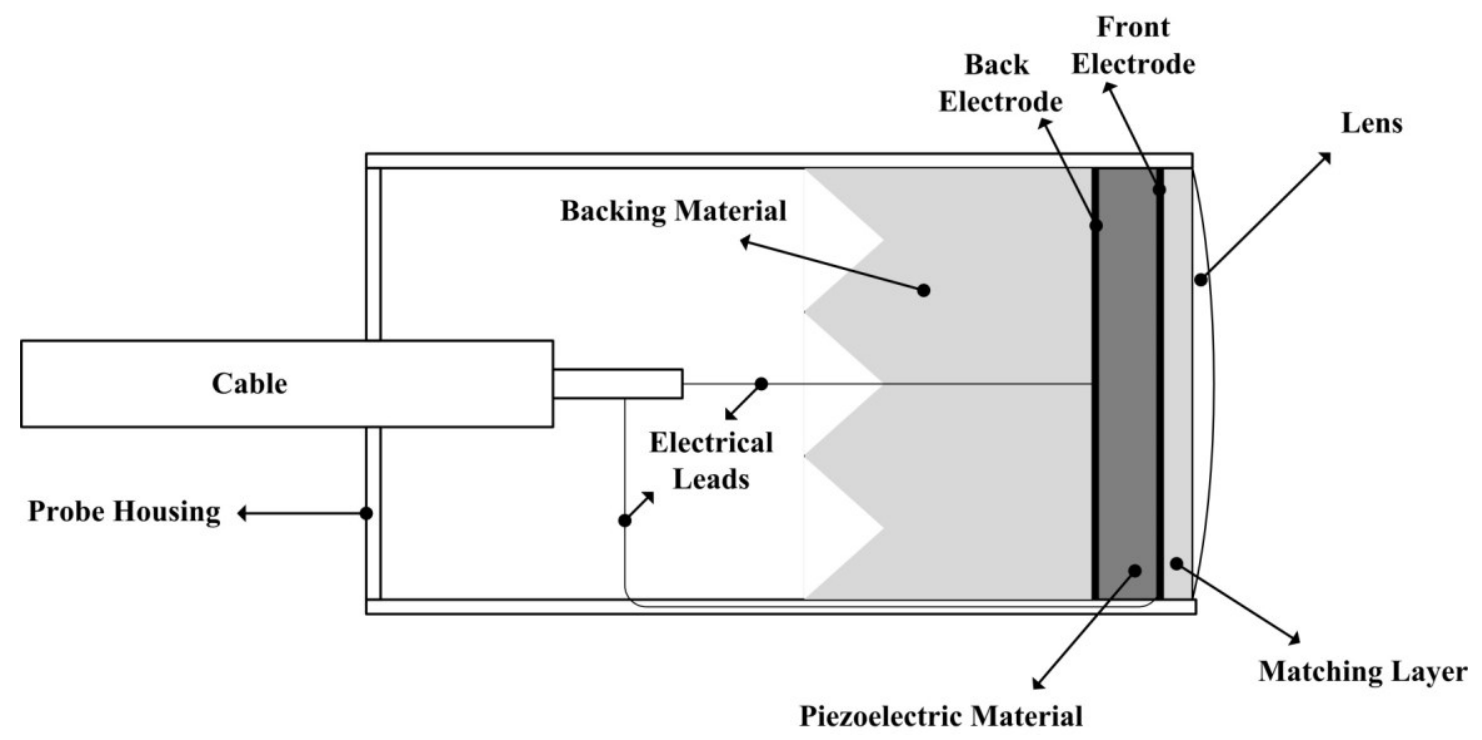

Figure 2.3: Schematic of basic ultrasonic probe with single transducer element. Based on [23], [28] 


\subsubsection{Electrodes}

The electrodes are two electrical connections that are formed on the front face and the back face of the piezoelectric material [27]. They are usually made of good conductive materials such as gold, silver or copper. The electrodes are connected to an ultrasonic power delivery system such as an ultrasonic pulser/receiver.

\subsubsection{Backing Material}

The backing material is attached to the back side of the piezoelectric material. It absorbs the backward travelling acoustic waves from the piezoelectric material in order to reduce the internal acoustic noise. It also has a damping effect which increases the frequency bandwidth of the ultrasonic pulsed signal to accomplish a high spatial resolution in ultrasonic measurements. The backing materials typically have an acoustic impedance matches the one of the piezoelectric film. They also have a high absorption coefficient to prevent the ultrasonic waves from re-entering the piezoelectric material [25].

\subsubsection{Matching Layer}

The matching layer is used to increase the transmission of the ultrasonic energy into the test subject. For instance, the ceramic piezoelectric material of PZT has an acoustic impedance about 20 times larger than human tissue. Thus, the majority of the ultrasound waves are reflected at a PZT-tissue interface [28] without a matching layer. The matching layer works to match the acoustic impedance of the probe to that of human 
tissue [25] in order to transmit more ultrasonic energy. More about acoustic impedance is described in Section 2.2.3.4.

\subsubsection{Probe Housing}

The probe housing seals all the individual components of the ultrasonic probe into one structure. Also, it provides the necessary structural support, protection, electrical insulation and radiofrequency shielding.

\subsubsection{Ultrasonic Coupling Medium}

A coupling medium or couplant is an agent that is usually applied between the ultrasonic sensor and the surface of the object to be measured. It eliminates the air gap therefore facilitates the ultrasonic wave passage into and out of the object. The existing of an air gab between the ultrasonic sensor and the object reflects all the ultrasound waves and prevents any ultrasonic penetration into the object [29]. In medical ultrasound, gel or liquid couplants are commonly applied to the skin before placing ultrasonic sensor.

\subsubsection{Portable Ultrasonic System}

Ultrasound has seen rapid growth in recent years and has become one of the preferred techniques in the medical field due to its low cost and non-ionizing radiation [30]. This growth of ultrasound applications and the requirement for periodic use of ultrasound technology have increased the demand for a miniaturized and portable 
ultrasonic system. ADR 2130 from Advanced Diagnostic Research Corporation (founded in 1972 in Tempe, Arizona, USA), shown in Figure 2.4(a), was one of the first portable ultrasound imaging units that was introduced commercially to the marketed in 1975 [31]. In 1978, Toshiba (Tokyo, Japan) also started marketing SAL-20A, shown in Figure 2.4(b), as a more portable compact diagnostic ultrasound system [31]. In the years following, the researchers have focused more on reducing the weight and the size of the ultrasonic system. Nowadays, many portable ultrasound systems are offered in a smaller size and lighter weight for a various number of applications such as NanoMaxx, shown in Figure 2.5(a), from FUJIFILM SonoSite (Bothell, Washington, USA) [32], Vscan Pocket Ultrasound, shown in Figure 2.5(b), from GE Healthcare (Little Chalfont, United Kingdom) [33], ACUSON P10, shown in Figure 2.5(c), from Siemens (Erlangen, Germany) [34], US Pro 2000, shown in Figure 2.5(d), from Current Solutions (Austin, Texas, USA) [35], and SeeMore Ultrasound Imaging USB probes, shown in Figure 2.5(e), from Interson Corporation (Pleasanton, California, USA) that can be Plugged into the USB port of a laptop, tablet, or desktop [36].

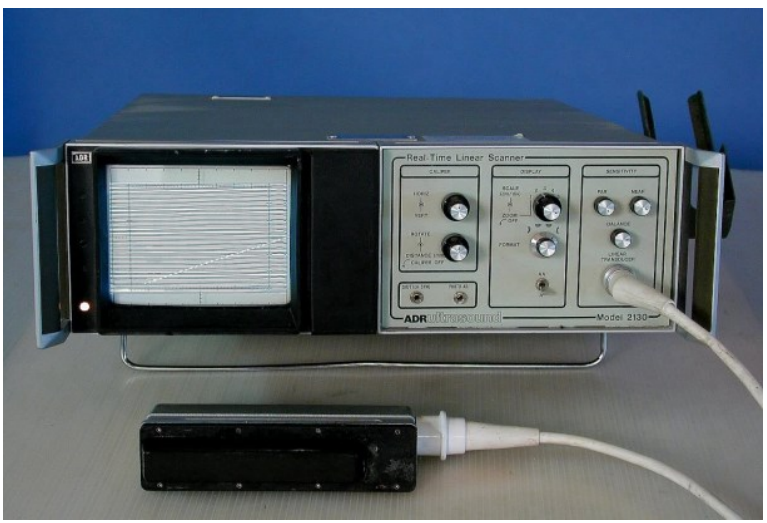

(a)

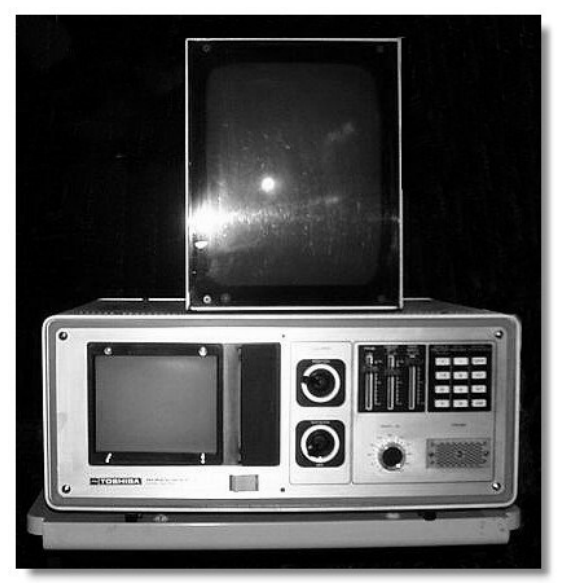

(b)

Figure 2.4: (a) ADR 2130, (b) Toshiba SAL-20A [31]. 


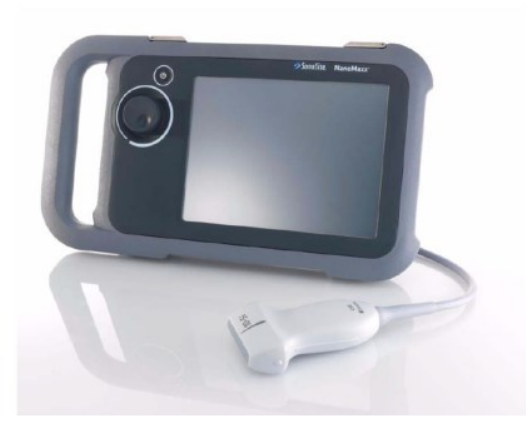

(a)

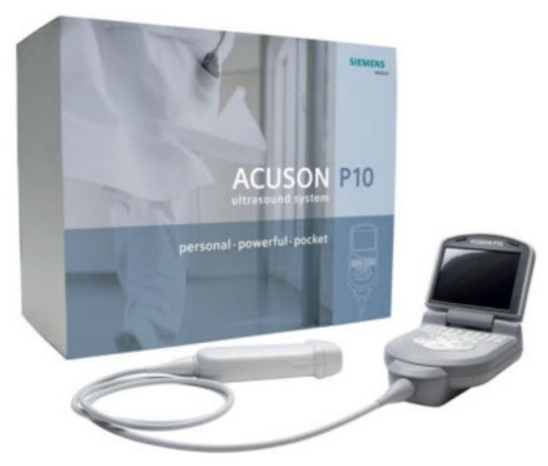

(c)



(b)

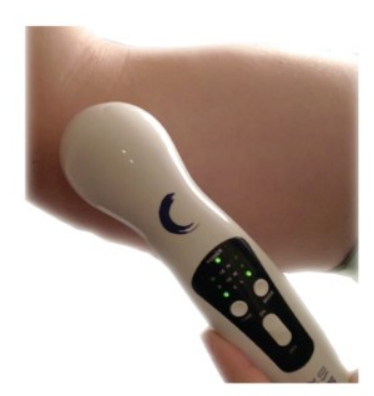

(d)

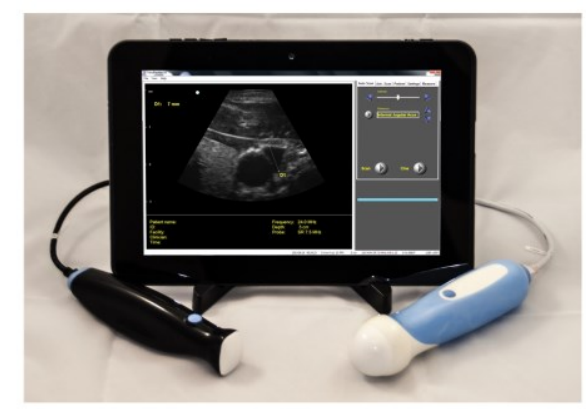

(e)

Figure 2.5: (a) SonoSite NanoMaxx [32], (b) GE Vscan [33], (c) Siemens ACUSON P10 [34], (d) Current Solutions US Pro 2000 [35], (e) Interson Corporation SeeMore Ultrasound Imaging USB probes [36].

Yet, many of the current medical ultrasonic probes are still handheld and bulky, and are not suitable to be worn on the human body. The interest in wearable sensors is 
fostered by several medical issues that require a free hand employing of the ultrasonic sensor and a constant monitoring of patients outside the hospital and clinics environment during the daily activities [37]. There are three main factors that should be considered for developing a wearable ultrasonic sensor: the size, the mechanical flexibility, and the weight. However, a number of studies have presented wearable ultrasonic sensors for several medical applications such as: monitoring of cardiac activity [38], [39], monitoring of fetal heart rate [40], [41], blood flow measurement [42], monitoring of urinary bladder volume [43], [44], monitoring of organ functions [45], and therapeutic ultrasound [46].

Rise et al. (1979) was one of oldest studies, to the best of our knowledge, that proposed a hand free ultrasonic sensor, shown in Figure 2.6(a), for bladder volume detection [43]. Rise et al. introduced an ultrasonic sensor with a $10 \mathrm{~mm}$ diameter made from a piezoelectric ceramic element that detects whether the bladder volume reached the threshold volume or not. Followed in 1993, Zuckerwar et al. developed a pressure sensor for fetal heart rate monitor that was made from PVDF piezoelectric polymer [40]. The sensor by Zuckerwar et al. was mounted on a belt that was worn by the mother and was sensitive in the audible range between $52.5 \mathrm{~Hz}$ and $2 \mathrm{KHz}$. Similar to Rise et al, Kristiansen et al. (2004) designed a new wearable ultrasonic bladder volume monitor, shown in Figure 2.6(b), that consists of seven phased array ultrasonic transducer arranged in a circular pattern [44]. The sensor developed by Kristiansen et al. may be considered bulky and rigid for other application such as muscle monitoring. However, Lanata et al. (2006) introduced a wearable ultrasound system for cardiac monitor using a sensor made from PVDF piezoelectric polymer, shown in Figure 2.6(c), [39]. The sensor design that was proposed by Lanata et al. is similar to the design of the developed sensor in this 
thesis. The sensor by Lanata et al. has dimensions of $10 \mathrm{~mm} \times 10 \mathrm{~mm}$ and was used to monitor the heart wall movement as it was wrapped around the chest. Bhuyan et al. (2011) also presented a wearable ultrasound probe, shown in Figure 2.6(d), that can be taped on the human body for constant monitoring of organ functions [45]. The active material of the sensor by Bhuyan et al. was developed using CMUT technology. The CMUT array was integrated on a flexible printed circuit board with total dimensions of $60 \mathrm{~mm} \times 35 \mathrm{~mm} \times 3.5 \mathrm{~mm}(\mathrm{~L} \times \mathrm{W} \times \mathrm{H})$. The acquired image by the CMUT sensor showed a comparable resolution to those of the commercially available probes. The sensor by Bhuyan et al. is relatively thick, which may limit its flexibility. Furthermore, a wireless and mobile system for fetal heart rate was introduced by Roham et al. (2011) [41]. The system consists of a wearable Doppler ultrasound sensor, shown in Figure 2.6(e), that was made from PZ-27 piezoelectric ceramic. The Doppler sensor by Roham et al. seems to be bulky and rigid due to its size and the active element material. On other hand, Huang et al. (2012) introduced a wearable Doppler ultrasonic sensor for blood flow measurement that was fabricated from flexible PMN-PT piezoelectric material, shown in Figure 2.6(f), [42]. The PMN-PT Doppler sensor has an aperture diameter of $4 \mathrm{~mm}$ and a thickness of $10 \mathrm{~mm}$. As the sensor was made from flexible active material, its flexibility may also be limited due to its thickness. Finally, Lewis et al. (2013) designed and constructed a wearable, battery operated therapeutic ultrasound device, shown in Figure 2.6(g), [46]. The 28-mm diameter and 4.8-mm thick wearable therapeutic sensor was constructed from PZT-4 piezoelectric ceramic and has a diverging lens. The thickness and active material of the sensor by Lewis et al. imply that the sensor lacks flexibility, which is a crucial feature for our proposed application in this thesis. 


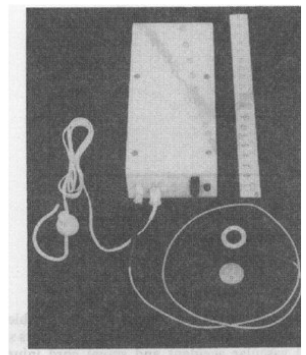

(a)

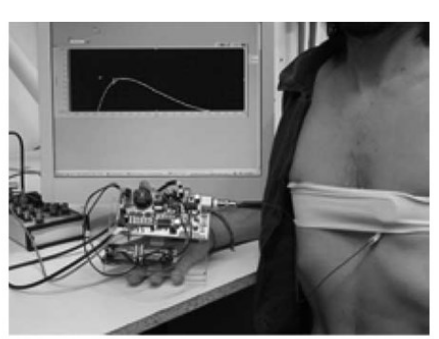

(c)

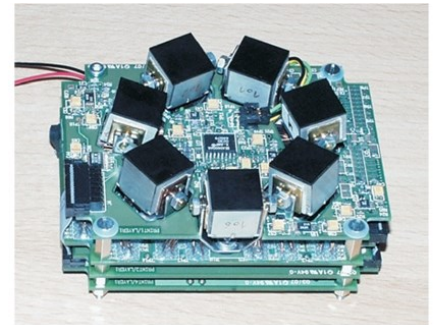

(b)

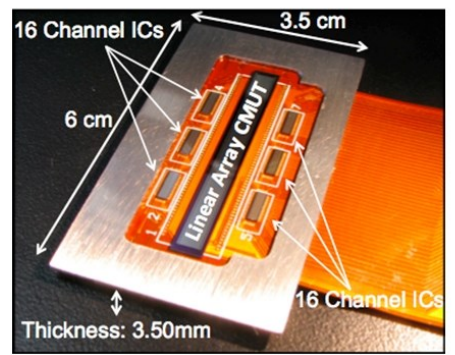

(d)

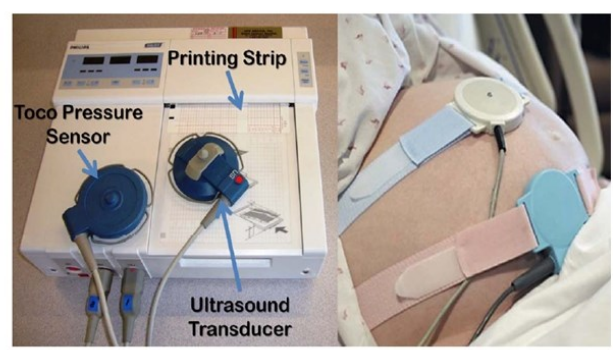

(e)

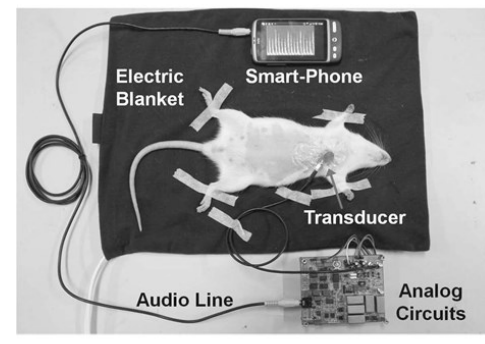

(f)

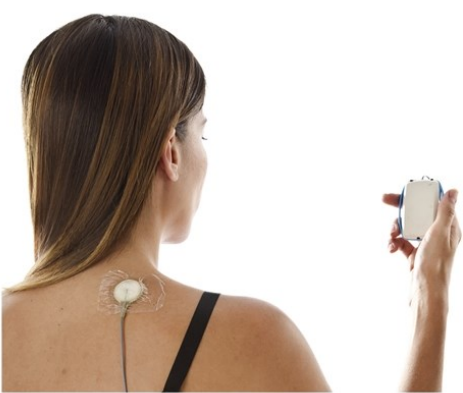

(g)

Figure 2.6: (a) Pocket-size ultrasonic bladder volume sensor [43], (b) wearable ultrasonic bladder volume [44], (c) wearable ultrasound for cardiac monitor [39], (d) wearable ultrasound probe [45], (e) wearable Doppler ultrasound [41], (f) ultrasonic surface transducer [42], and (g) therapeutic ultrasound device [46] 
Table 2.1: Ultrasonic sensor summary.

\begin{tabular}{|c|c|c|c|}
\hline Ultrasonic sensor & Active element & $\begin{array}{l}\text { Dimensions } \\
(\mathrm{mm})\end{array}$ & $\begin{array}{l}\text { Attachment } \\
\text { method }\end{array}$ \\
\hline $\begin{array}{l}\text { Conventional } \\
\text { medical imaging } \\
\text { probe }\end{array}$ & $\begin{array}{l}\text { Commonly } \\
\text { piezoelectric } \\
\text { Ceramic }\end{array}$ & $\begin{array}{c}\text { Various sizes } \\
\text { Example: Esaote LA40 [47] } \\
15 \text { (Width) x } 55 \text { (Length) } \\
\times 100 \text { (Height) }\end{array}$ & Hand \\
\hline $\begin{array}{l}\text { Conventional single } \\
\text { element transducer }\end{array}$ & $\begin{array}{l}\text { Commonly } \\
\text { piezoelectric } \\
\text { Ceramic } \\
\text { composite }\end{array}$ & $\begin{array}{c}\text { Various sizes } \\
\text { Example: Olympus Fingertip } \\
\text { Contact }[48] \\
6 \text { (Diameter }) \times 9.7 \text { (Height) }\end{array}$ & Hand \\
\hline $\begin{array}{l}\text { Wearable transducer } \\
\qquad[44]\end{array}$ & PZT & $\begin{array}{c}16 \text { (Width) } \times 16 \text { (Length) } \times 15 \\
(\text { Height })\end{array}$ & Belt \\
\hline $\begin{array}{l}\text { Wearable ultrasound } \\
\qquad[39]\end{array}$ & PVDF & $\begin{array}{c}10 \text { (Width) } \times 15 \text { (Length) } \times 5.0 \\
(\text { Height })\end{array}$ & Belt \\
\hline $\begin{array}{l}\text { Wearable ultrasound } \\
\text { probe }[45]\end{array}$ & CMUT & $\begin{array}{c}35 \text { (Width) } \times 60 \text { (Length) } \times 3.5 \\
(\text { Height })\end{array}$ & Tape \\
\hline $\begin{array}{l}\text { Wearable Doppler } \\
\text { ultrasound [41] }\end{array}$ & PZ-27 & Not provided & Belt \\
\hline $\begin{array}{l}\text { Ultrasonic surface } \\
\text { transducer [42] }\end{array}$ & PMN-PT & 4.0 (Diameter), 10 (Height) & Tape \\
\hline $\begin{array}{l}\text { Therapeutic sensor } \\
{[46]}\end{array}$ & PZT-4 & $\begin{array}{c}28 \text { (Diameter), } 4.8 \mathrm{~mm} \\
\text { (Height) }\end{array}$ & Tape \\
\hline $\begin{array}{l}\text { Wearable sensor } \\
\text { (this thesis work) }\end{array}$ & PVDF & $\begin{array}{c}5.0 \text { (Width) x } 5.0 \text { (Length) } \\
\text { and up, } 0.2 \text { (Height) }\end{array}$ & Tape \\
\hline
\end{tabular}




\subsubsection{Ultrasound Physics}

Ultrasound technology is governed by the physics of sound. In order to convey a general understanding of the ultrasound principle, the following subsections provide a brief overview of some basic concepts of ultrasonic sensor properties and acoustic wave propagation.

\subsubsection{Intensity}

The intensity of an ultrasound is defined as the amount of energy per unit area per second. It is expressed in the unit of watts per square meter [25]. The ultrasound intensity is proportional to the square of the pressure amplitude. Thus, the time-averaged intensity is given by [25]:

$$
I_{A}=\frac{p_{o}^{2}}{2 \rho v}
$$

where $p_{o}$ is the maximum-pressure, $v$ the velocity of sound in the medium, $\rho$ the density of the medium.

For an ultrasonic sensor, the intensity of a transmitted ultrasound depends partly on the applied electrical energy. Increasing the applied voltage increases the amplitude of the piezoelectric oscillation. Moreover, the safety of medical ultrasound transducers to biological tissue is regulated according to the parameters of the acoustic output intensities and indices [29], [30]. These parameters are: the spatial peak pulse average intensity $\left(I_{S P P A}\right)$, the spatial peak temporal average $\left(I_{S P T A}\right)$, the mechanical index $(\mathrm{MI})$ and the thermal index (TI) [49], [50]. 
In ultrasound measurement and imaging for medical applications, the intensity is commonly determined using relative measurements due to the difficulty of measuring the absolute values of the ultrasound intensity and lack of the necessity for absolution intensity measurement. Relative measurement compares the value at one point with a reference intensity at another point. Thus, the intensity level is given in decibel (dB) by [25]:

$$
\operatorname{Level}(\mathrm{dB})=10 \log \left(\frac{I_{U S}}{I_{\text {ref }}}\right) \text {, }
$$

where $I_{U S}$ is the intensity at the point of interest, and $I_{r e f}$ is the reference intensity.

\subsubsection{Resonant Frequency}

The resonant frequency is the natural vibration frequency of the piezoelectric material. For a piezoelectric film vibrating in thickness-extensional (TE) mode, the resonant frequency is determined thickness of the piezoelectric film. The relation between the resonant frequency and the piezoelectric material thickness can be expressed as [23]:

$$
f_{o}=\frac{v}{2 d_{F}}
$$

where $\mathrm{f}_{\mathrm{o}}, \mathrm{v}, \mathrm{d}_{F}$ are the fundamental resonant frequency, sound velocity and thickness of the piezoelectric film, respectively. However, it is noted that for the pulsed system, the ultrasonic sensor generates an ultrasound with a range of frequencies [25] because of the pulse excitation and the damping mechanism of an ultrasonic sensor. The higher 
ultrasonic frequencies generally provide better spatial resolution but less penetration depth due to the greater attenuation.

\subsubsection{Attenuation}

Ultrasound attenuation is the reduction of acoustic wave energy as it propagates through the medium. The factors that cause the attenuation during wave propagation include absorption, scattering, diffraction and mode conversion [23]. Absorption is the process whereby the acoustic energy is transformed to thermal energy, which is then dissipated in the medium. Scattering is the interaction of ultrasound with small reflecting targets inside the medium that causes a scattering of ultrasound over a large range of angles [28]. Diffraction is the divergence of the ultrasound beam as the ultrasonic waves propagates further from the source [25]. Diffraction also is the bending of ultrasonic waves around small obstacles and the spreading out of waves past small openings. Mode conversion is the processes by which longitudinal waves are converted to transverse shear waves [23]. Attenuation is considered a limiting factor on the depth of ultrasound penetration into an object of interest. It also, increases with the ultrasonic frequency employed.

\subsubsection{Acoustic Impedance}

Acoustic impedance, $Z$, is the resistance to ultrasound passing through the medium [25]. It is equal to the density of a medium multiplied by its sound speed and expressed as kilograms per square meter per second $\left(\mathrm{kg} / \mathrm{m}^{2} / \mathrm{s}\right)[29]$. 


$$
Z=\rho v
$$

\subsubsection{Reflection and Transmission}

Reflection is the major feature of interest in ultrasound measurement and imaging. It occurs at the interface of two media or tissue boundaries where there is a difference in acoustic impedance between the two media. When the ultrasound waves travel from one type of medium or tissue to another type with a different acoustic impedance, some of the

ultrasound energy is reflected back toward the source of the wave (the echo) [28]. The portion of the reflected ultrasound wave increases with a greater acoustic impedance difference at the interface. The reflectivity of ultrasound propagation between two media is given by the amplitude reflection coefficient, $A R C$, [29]

$$
A R C=\frac{Z_{2}-Z_{1}}{Z_{2}+Z_{1}}
$$

where $Z_{2}$, is the acoustic impedance of the second medium (distal to the interface); $Z_{1}$ is the acoustic impedance of the first medium (proximal to the interface). The intensity transmittivity of ultrasound propagation into the second medium is given by the amplitude transmission coefficient, $A T C$,

$$
A T C=\frac{2 Z_{2}}{Z_{2}+Z_{1}} .
$$




\subsection{Ultrasound Measurement System}

The ultrasound system used in this thesis consists of an ultrasonic sensor (detailed discussion in Chapter 3 and 4), an ultrasonic pulser/receiver, a digitizer and a personal computer. The configuration of the connections of the system components employed in the experiments for this thesis research are shown in Figure 2.7 for the ultrasonic pulse-echo technique and Figure 2.8 for the ultrasonic through-transmission technique.

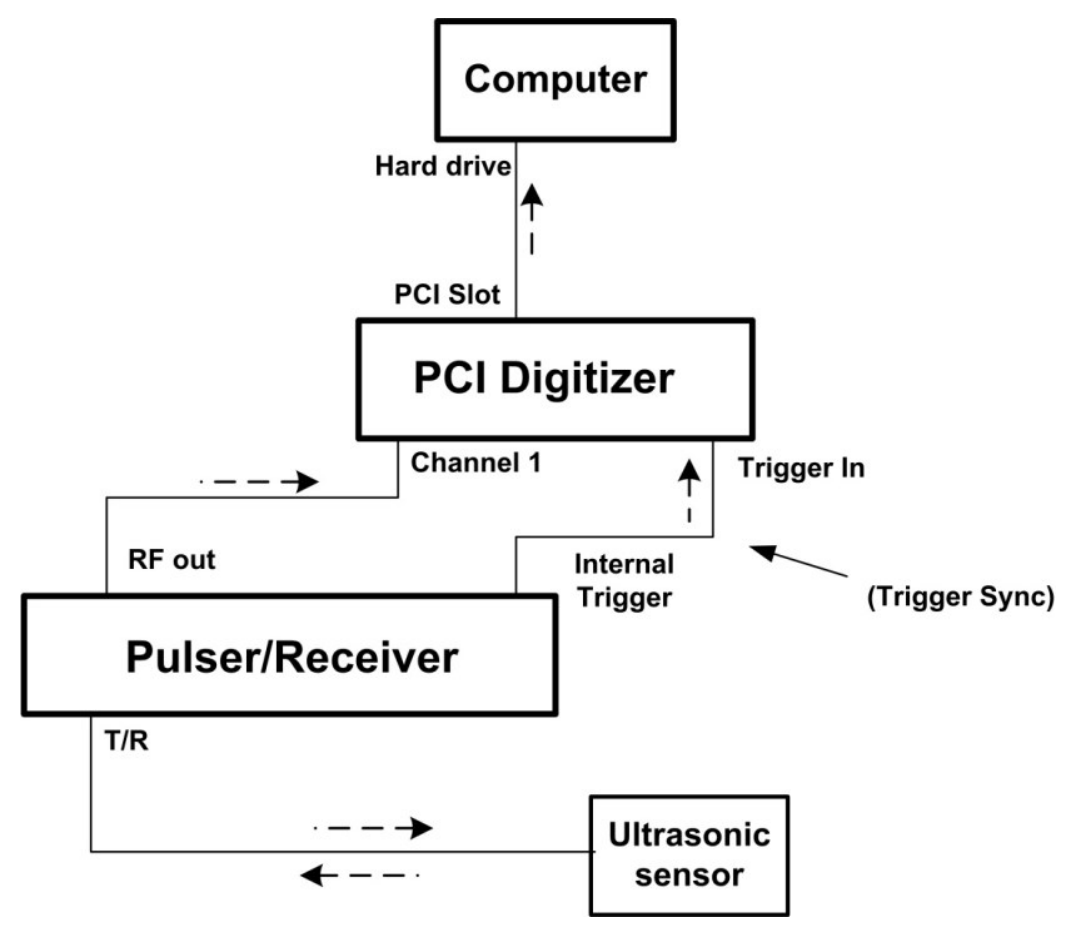

Figure 2.7: Ultrasound System Connection for the pulse-echo technique. 


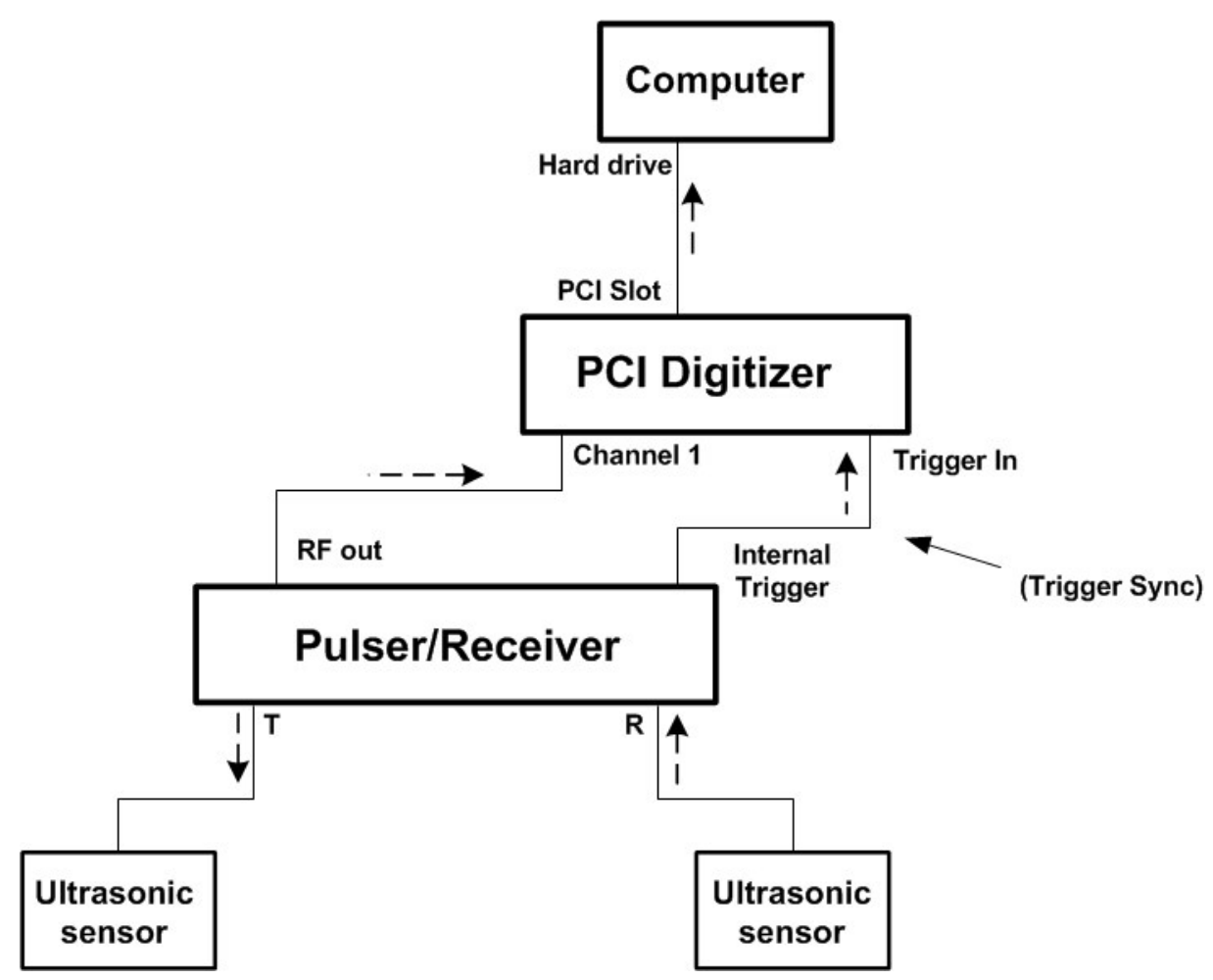

Figure 2.8: Ultrasound System Connection for the through-transmission technique.

\subsubsection{Ultrasonic Pulser/Receiver}

The ultrasonic pulser/receiver produces electric pulses that drive the ultrasonic sensor to generate a pulse ultrasonic wave, and receives the electric signal back from the sensor. In this research, two pulser/receivers have been used: NDT 5900PR Model from Panametrics-Olympus (Waltham, MA, USA) and DPR 300 Model from JSR Ultrasonics (Pittsford, NY, USA), as shown in Figure 2.9. 


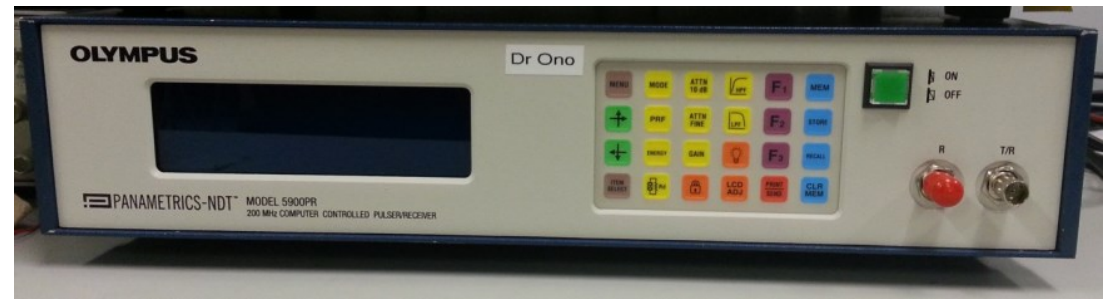

(a)

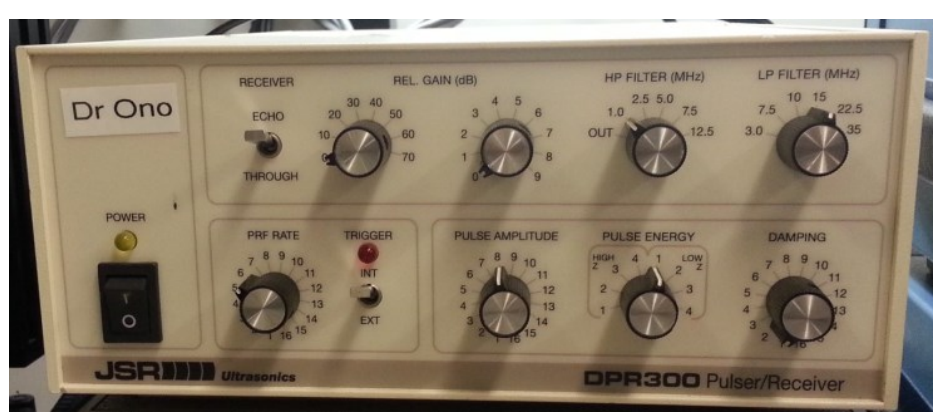

(b)

Figure 2.9 : Ultrasonic pulser/receiver devices: (a) NDT 5900PR, (b) DPR 300.

\subsubsection{Data Acquisition System}

The data acquisition (DAQ) system consists of two parts: PCI digitizer and DAQ software. For this thesis research, the ATS 460 Model from AlazarTech (Montreal, QC, Canada), shown in Figure 2.10, was used to acquire the signals from the ultrasonic pulser/receiver. It has a 14-bit resolution and a sampling rate range from $10 \mathrm{KHz}$ to 125 MHz. The DAQ software was programmed by LABVIEW platform. It collects and saves the data into the storage of a PC. The ultrasonic data are analyzed by a signal processing program by MATLAB. 

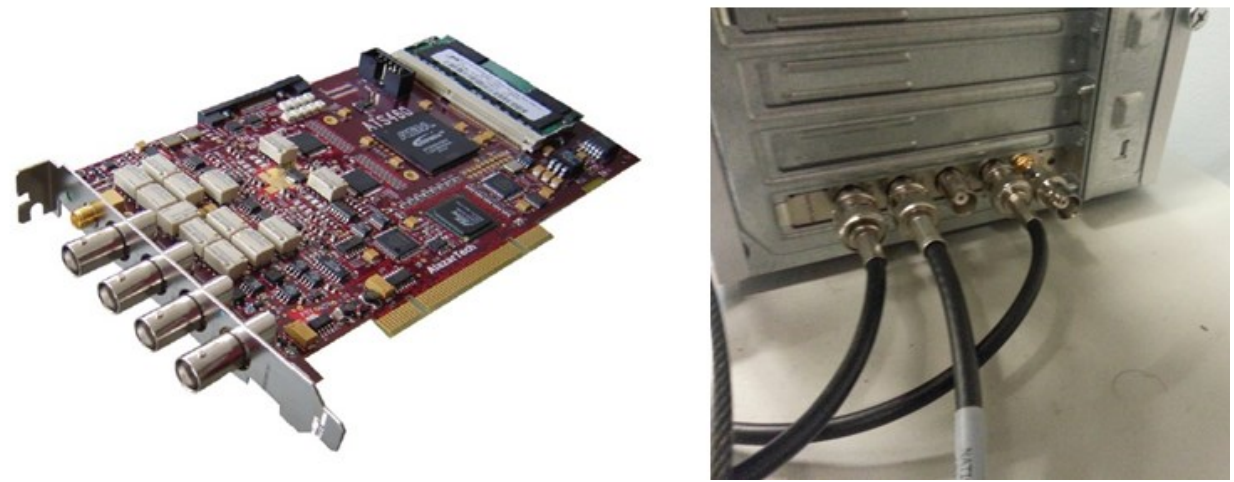

Figure 2.10: PCI Digitizer [63]. 


\section{Chapter 3: Sensor Development}

The goal of this thesis is to develop an ultrasonic sensor that can be worn over an area of interest on the body surface to monitor muscle contraction with hands-free interaction. There are a number of considerations that should be taken into account when designing such a wearable ultrasonic sensor.

One consideration is the size of the sensor in relation to the area in which it will be applied. The sensor must also be flexible enough to fit and bend over the uneven and rounded nature of the human body surface. In addition, movement of the underlying tissue during contraction must not be restricted or limited by the attachment of the sensor. Therefore, the sensor should be light enough so that its mass-loading effect on underlying tissue motion can be neglected. Furthermore, the acoustic impedance, ultrasonic frequency and bandwidth of the sensor should be well suited for operation on biological tissues.

\subsection{Piezoelectric Material Selection}

There are a variety of piezoelectric materials used for ultrasonic sensors such as crystals, ceramics, polymers and their composites. These materials vary in their piezoelectric activities and properties. Choosing a material for such a workable ultrasonic sensor should meet the considerations that were previously discussed. Generally in medical applications, piezoelectric ceramics such as lead zirconate-titanate (PZT) are 
most commonly used due to their high electromechanical coupling, wide selection of dielectric constants and low electrical and mechanical losses [51], [52]. However, the high acoustic impedance (PZT: $32 \times 10^{6} \mathrm{~kg} / \mathrm{m}^{2} \mathrm{~s}$ [53]) comparable to the acoustic impedance of human tissue $\left(1.63 \times 10^{6} \mathrm{~kg} / \mathrm{m}^{2} \mathrm{~s}\right.$ [54]) and the brittle form (nonflexibility) of the ceramics are the major drawbacks as a piezoelectric material for a wearable and flexible ultrasonic sensor for our application of muscle monitoring.

On the other hand, piezoelectric polymers, such as polyvinylidene fluoride (PVDF) and polyvinylidene fluoride trifluoroethylene [P(VDF-TrFE)], offer a high flexibility and an acoustic impedance that better matches that of human tissues, when compared to ceramics. Also, they can easily be fabricated into any desired shapes. These advantages outweigh their relatively weak piezoelectric effect. Table 3.1 lists the electromechanical properties of PVDF, P(VDF-TrFE), and ,for comparison, PZT. It worth noting that the negative sign of the piezoelectric $e$ and the transmitting constant $h$ of the PVDF and the P(VDF-TrFE) indicates that they generate tensile strain (stretch) with a negative electric filed.

$\mathrm{P}(\mathrm{VDF}-\mathrm{TrEF})$ has superior electricomechanical coupling and smaller dielectric and mechanical losses among polymers (details will be discussed in Chapter 4). However, PVDF offers more flexibility and greater compliance with satisfying overall piezoelectric properties [55]. It is also commercially available in the form of metalized film sheet. For these reasons, PVDF was chosen in this thesis study as a piezoelectric material to develop a wearable and flexible ultrasonic sensor. 
Table 3.1: Electromechanical properties of some piezoelectric materials [6].

\begin{tabular}{|l|c|c|c|}
\hline Properties & PVDF & P(VDF-TrFE) & PZT \\
\hline $\begin{array}{l}\text { Density } \rho \\
\left(10^{3} \mathrm{~kg} / \mathrm{m}^{3}\right)\end{array}$ & 1.78 & 1.88 & 7.5 \\
\hline $\begin{array}{l}\text { Longitude Sound Velocity } v \\
\left(10^{3} \mathrm{~m} / \mathrm{s}\right)\end{array}$ & 2.26 & 2.40 & 4.63 \\
\hline $\begin{array}{l}\text { Acoustic Impedance } \\
\left(10^{6} \mathrm{~kg} / \mathrm{m}^{2} \mathrm{~s}\right)\end{array}$ & 4.02 & 4.51 & 34.4 \\
\hline $\begin{array}{l}\text { Coupling Factor } k_{t} \\
\text { Piezoelectric Constant } e \\
\left(\mathrm{C} / \mathrm{m}^{2}\right)\end{array}$ & -0.14 & -0.22 & 15.1 \\
\hline $\begin{array}{l}\text { Elastic Stiffness } c^{D} \\
\left(10^{9} \mathrm{~N} / \mathrm{m}^{2}\right)\end{array}$ & 9.1 & 11.3 & 159 \\
\hline $\begin{array}{l}\text { Dielectric Permittivity } \varepsilon^{s} / \varepsilon_{o} \\
\text { Dielectric Loss Tangent } \\
\text { tan } \delta_{e}\end{array}$ & 6.2 & 5.3 & 6.31 \\
\hline $\begin{array}{l}\text { Mechanical Loss Tangent } \\
\text { tan } \delta_{m}\end{array}$ & 0.10 & 0.04 & 0.35 \\
\hline $\begin{array}{l}\text { Transmitting Constant } h \\
\left(10^{9} \mathrm{~V} / \mathrm{m}\right)\end{array}$ & -2.6 & -4.7 & 0.004 \\
\hline
\end{tabular}




\subsection{Developed Sensor Construction}

In this section the fabrication procedure for the development of the sensor is discussed in detail. The structure of the developed ultrasonic sensor consists of three main layers: the piezoelectric material, the electrodes and the protection layer. The schematic showing the basic structure of the developed sensor is presented in Figure 3.1. The total thickness and weight of the constructed sensor are $200 \mu \mathrm{m}$ and $0.3 \mathrm{~g}$ respectively. Thus, it can easily be worn and bended due to its high flexibility and thickness, as shown in Figure 3.2.

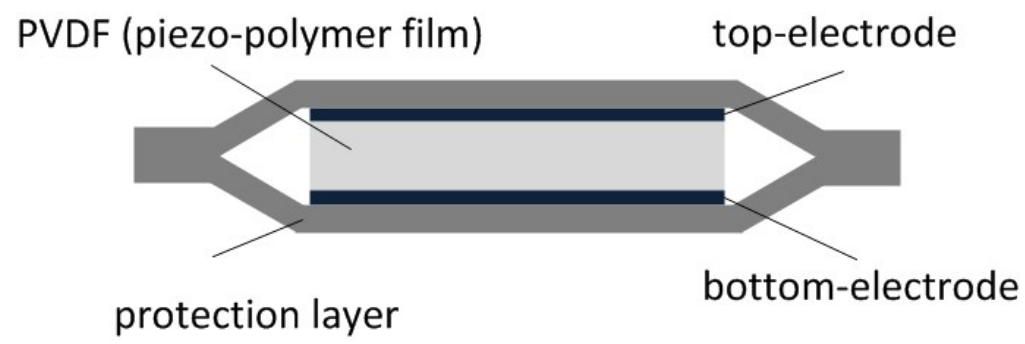

Figure 3.1: schematic representation of the ultrasonic sensor design

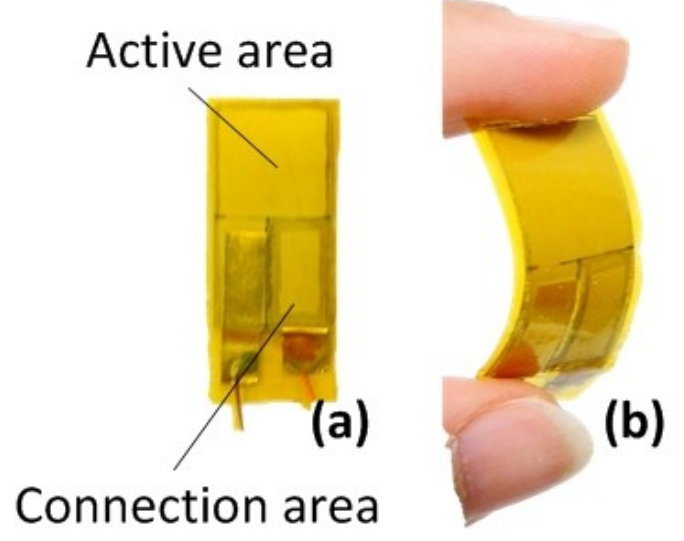

Figure 3.2: photographs of the ultrasonic film sensor developed $(1.5 \mathrm{~cm} \times 1.5 \mathrm{~cm}$ active area): (a) top view and (b) side view while bending. 


\subsubsection{Piezoelectric Film}

PVDF was chosen as a piezoelectric material for the construction of the sensor due to its inherent properties that meet the requirements of a wearable sensor, such as flexibility, light weight and low acoustic impedance. The PVDF films were obtained from Measurement Specialties Inc. (Hampton, USA) in the form of metallized film sheet as shown in Figure 3.3. PVDF operates in thickness mode when it is used as an ultrasonic sensor. Thus, the resonance frequency of the film depends on the thickness of the film. Figure 3.4 shows the calculated PVDF fundamental resonant frequency, $f_{o}$, for film thicknesses ranging from 20 to $200 \mu \mathrm{m}$ based on equation (2.3) (given in Chapter 2). PVDF resonance ranges from a high resonance frequency for thinner films $(65.5 \mathrm{MHz}$ at $20 \mu \mathrm{m}$ thick) to a low resonance frequency for thicker films (5.65 MHz at $200 \mu \mathrm{m}$ thick). For the purpose of our application, a 110- $\mu \mathrm{m}$ thick film was used to design the sensor due to its suitable operating resonance frequency, as will be discussed in more detail in Chapter 5.

The metalized PVDF films were provided by the vender in a large sheet size (20.32 x $27.94 \mathrm{~cm})$, and can be cut into any desired sizes. The size of the overlapped electrodes on the film determines the active area size of the sensor where ultrasound is excited and transmitted. Therefore, several single element sensors were constructed in different sizes $\left(0.25,1.00,2.25,4.00\right.$ and $\left.6.25 \mathrm{~cm}^{2}\right)$ based on the area intended to be measured (more detailed discussion in Chapter 5).

The film preparing procedure starts with handling the film carefully by avoiding any tear or scratch to the PVDF film and electrode layers. Then, using a sharp knife, the film is cut into the desired shape and dimensions. 




Figure 3.3: Metallized PVDF film sheet (silver paint electrode).

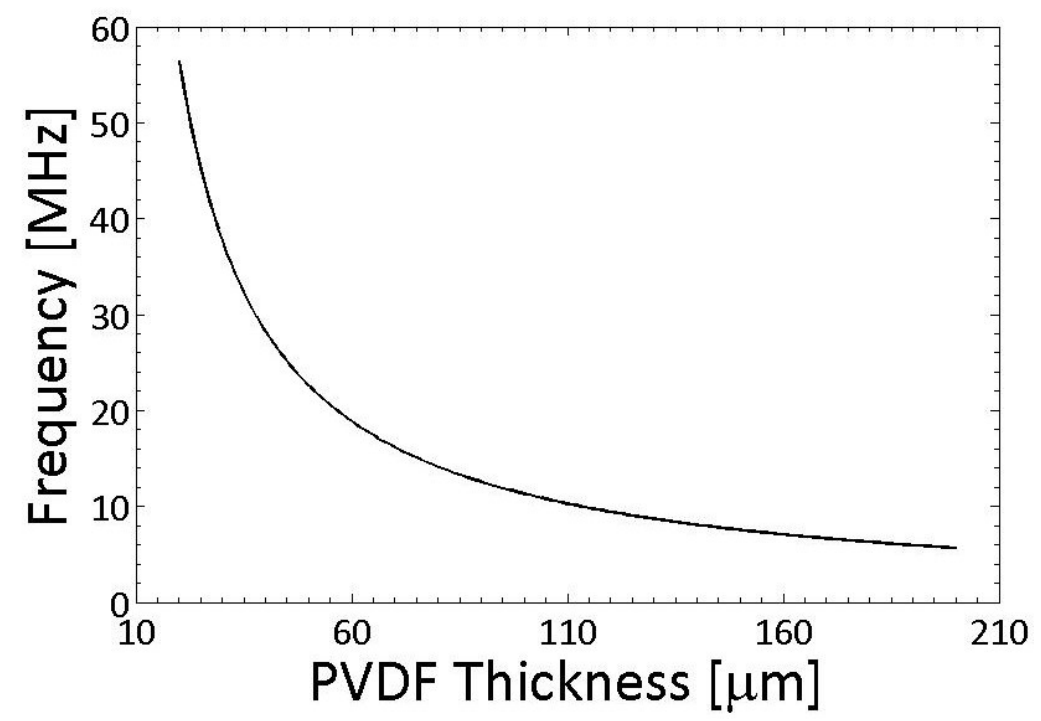

Figure 3.4: PVDF resonance frequency with respect to PVDF thickness. 


\subsubsection{Lead Wire Connection}

Two PVDF films with different electrode materials were used to fabricate the sensors. One film is sandwiched by top and bottom silver paint electrodes, and the other is sandwiched by top and bottom copper-nickel $(\mathrm{Cu}-\mathrm{Ni})$ electrodes. Figure 3.5 shows the schematic of the PVDF film with the electrode layers. The silver paint electrode is $13 \mu \mathrm{m}$ thick (total of $26 \mu \mathrm{m}$ for both sides) and the copper-nickel is $0.035 \mu \mathrm{m}$ thick (total of 0.07 $\mu \mathrm{m}$ for both sides). The reason for choosing these types of electrodes is to observe the electrodes mass-loading effect on the PVDF ultrasonic performance (discussed in chapter 5) since the $\mathrm{Cu}-\mathrm{Ni}$ layer is relatively thin and light so its mass-loading on $110-\mu \mathrm{m}$ PVDF film can be neglected.

After cutting the PVDF film into the desired size, the electrode layer around the edges was removed about $1 \mathrm{~mm}$ from the edge in order to prevent electrical shorting between the top and bottom electrodes. Also, the film was divided into two parts: the active area and the interconnection area, as shown in Figure 3.6. The active area is the functional part of the sensor where the top and bottom electrodes overlap to transmit and receive ultrasound. The interconnection area is the part where the lead wires attach to the electrodes, and there is no overlap of the top and bottom electrodes.

Several approaches were attempted to achieve a reliable lead wire connection to the PVDF film. One was a piercing technique where the ring tongue terminal and the wire were mechanically pressed against the film by a rivet through a hole. The major drawback of this technique was the pressure exerted by the rivet which limits the film flexibility. In the other technique, a conductive silver epoxy was used to glue the wire on the electrode surface, but it was found that the conductive epoxy doesn't adhere well with 
the electrode surface and increases the electrical noise level. However, attaching the wire directly on the electrode surface was found to be a simple and effective technique. In this technique, the wire was soldered to a piece of shim $(12-\mu \mathrm{m}$ thick metal sheet $)$ of the same size of the interconnection area. It was then affixed directly to the electrode surface by a tape (explained in the next section). The tape was used to wrap the wires of the top and bottom electrode together, along with the film. This approach preserves the simplicity and flexibility of the sensor and is found to be an appropriate lead attachment method for our design.

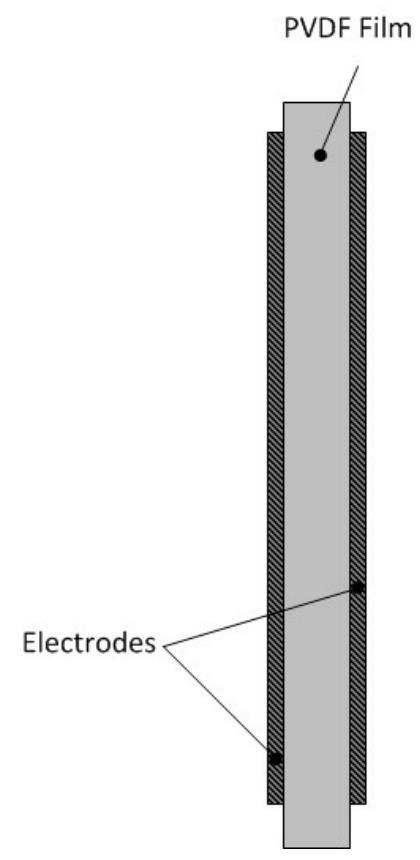

Figure 3.5: PVDF film sandwiched by top and bottom electrodes. 


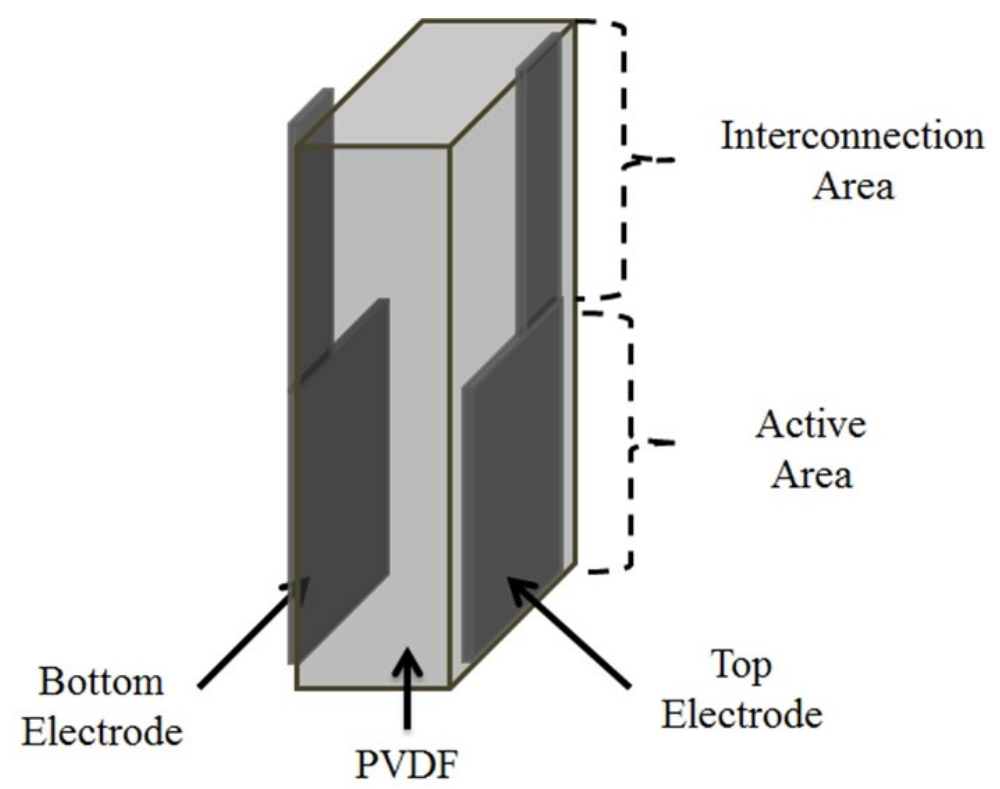

Figure 3.6: The active and the interconnection area of the developed sensor.

\subsubsection{Protection Layer}

The constructed sensor required an additional layer to provide protection and electrical insulation, and to waterproof the PVDF film and the leads attachment. Also, it was needed to affix the lead wires on the electrodes. The first attempt at constructing the protection layer was with an epoxy. A two parts epoxy (adhesive and hardener) was mixed together, then a thin layer of the epoxy mixture was applied to the entire structure of the sensor so that after the epoxy cured, the sensor would be sealed by the epoxy layer. However, the precise metering of a thin layer of epoxy was rather difficult and messy. Instead, the use of an adhesive tape was found to be a more practical approach. The tape was applied carefully on both sides of the sensor with gentle pressure, in order to avoid any air gap between the film and the tape. Several adhesive tape products have been evaluated as protection layer, for instance packing tape, scrapbooking tape, transparent 
tape, stationary tape and polyimide tape. Polyimide tape among others was chosen due to a number of features.

Polyimide materials are flexible, lightweight and have good chemical resistance. Also, they are excellent electrical insulators, and have low acoustic impedance and attenuation coefficient. In addition, polyimide tapes are commercially available in various thicknesses, with an adhesive layer.

13- $\mu \mathrm{m}$ thick polyimide tape with a silicone adhesive was used as a protection and isolation layer for the sensor. The strong silicone adhesive $(1.4 \mathrm{~N} / \mathrm{cm})$ ensures the longterm stability of the tape, especially for the lead wire interconnection. 


\section{Chapter 4: Numerical Simulation Model}

This chapter deals with the numerical simulation model of the developed wearable ultrasonic sensor. The simulation model is for the theoretical calculation of the developed sensor's performance. The mathematical representation of the developed sensor with the presented multilayer structure is also discussed. It is based on Mason's electric equivalent circuit model of piezoelectric resonators [56]. In addition, the internal losses of piezoelectric polymers have been considered in the expression of the electromechanical properties of the sensor as proposed by Ohigashi et al [57], for accurate prediction of its ultrasonic performance. The numerical simulation helps extend the investigation of the sensor's performance beyond the limitations of the experimental conditions and validate the experimental results.

\subsection{Mathematical Expression of the Sensor}

Polymer piezoelectric materials have large dielectric and mechanical losses compared to ceramic piezoelectric materials. For example, the dielectric and mechanical loss tangents of the PVDF polymer piezoelectric are 0.25 and 0.1 , respectively, while they are 0.004 and 0.004 for the PZT ceramic piezoelectric. Therefore, the electric energy applied to the piezoelectric polymers is partially converted into acoustic output energy, and partially lost in the form of internal thermal dissipation. The amount of thermal energy dissipated is equal to and even often larger than the acoustic energy [57]. For this 
reason, the internal energy loss must be taken into account for an accurate numerical calculation of the piezoelectric polymer sensor characteristics. In the following sections, the mathematical expression of the electromechanical characteristics of the developed ultrasonic sensor composed of piezoelectric and non-piezoelectric layers will be derived.

\subsubsection{Piezoelectric Layer}

In the 1940s, Mason's derivation of an equivalent circuit was introduced to analyze the electromechanical behavior of piezoelectric resonators [56]. Since then, numerous circuit models have been used to study piezoelectric ceramics transducers, but the internal losses were neglected due to their very small effect [55], [58]. However, analyzing the electromechanical behavior of piezoelectric polymers using Mason's equivalent circuit without considering the internal losses brings large errors in the resulting values. Therefore, Ohigashi and others introduced complex components into the piezoelectric coefficients for piezoelectric polymer transducers [57], [59]. They introduced the mechanical loss factor, $\tan \delta_{m}$, into the elastic stiffness constant, $c^{*}$, and the dielectric loss factor, $\tan \delta_{e}$, into the dielectric permittivity, $\varepsilon^{*}$, [59]:

$$
\begin{aligned}
c^{*} & =c^{D}\left(1+j \tan \delta_{m}\right), \\
\varepsilon^{*} & =\varepsilon^{S}\left(1-j \tan \delta_{e}\right),
\end{aligned}
$$

where $c^{D}$ is elastic stiffness at constant electric displacement, and $\varepsilon^{S}$ is dielectric permittivity at constant strain.

The electromechanical dynamic behavior of a piezoelectric, vibrating in the thickness extensional (TE) mode, can be described by a three ports network [53], [60], as shown in Figure 4.1. There are two acoustic ports and one electric port. The acoustic 
ports are represented by the force $F_{1}$ and $F_{2}$ acting on each surface of the film, and the particle velocity $U_{1}$ and $U_{2}$ for each surface. The electric port is represented by the voltage $V$ across the film, and the current $I$ flowing across the film. From Figure 4.1, $F_{1}$ and $F_{2}$ are expressed as [57]:

$$
F_{1}=-A T(0), F_{2}=-A T(d)
$$

where $A$ is the area of the film surface and $T$ is the internal stress. $U_{1}$ and $U_{2}$ are the velocity at $z_{x}=0$ and $z_{x}=d$, respectively, where $z_{x}$ is an axis along the film thickness and $d$ is the film thickness.

The force $F$ and the velocity $U$ are equivalent to voltage $V$ and current $I$ in the electrical circuit, respectively. The relationships between the $V, F_{1}, F_{2}, I, U_{1}$ and $U_{2}$ are expressed in a $3 \times 3$ matrix form as [57], [59] :

$$
\left(\begin{array}{c}
F_{1} \\
F_{2} \\
V
\end{array}\right)=-j\left(\begin{array}{ccc}
B \operatorname{coth} \gamma d & B \operatorname{csch} \gamma d & h / \omega \\
B \operatorname{csch} \gamma d & B \operatorname{coth} \gamma d & h / \omega \\
h / \omega & h / \omega & 1 / C \omega
\end{array}\right)\left(\begin{array}{c}
U_{1} \\
U_{2} \\
I
\end{array}\right)
$$

where $B$ is the acoustic impedance for an area of the film surface, and is defined as:

$$
B=j \rho v\left(1+j \tan \delta_{m}\right) A
$$

where $\rho$ is the density of the piezoelectric film, and $v$ is the longitudinal velocity of the sound in the piezoelectric film. Also, $C$ is the capacitance of the sensor that is defined as: [57], [59]

$$
C=\frac{\varepsilon^{S}\left(1-j \tan \delta_{e}\right) A}{d}=\frac{\varepsilon^{*} A}{d}
$$

$\gamma$ is expressed as:

$$
\gamma=j\left(\frac{\omega}{v}\right)\left(1-\frac{j \tan \delta_{m}}{2}\right)
$$


where $\omega$ is the angular frequency. Finally, $h$ is the transmitting constant that is expressed as: [60]

$$
h=\frac{e}{\varepsilon^{S}},
$$

where $e$ is the piezoelectric constant.

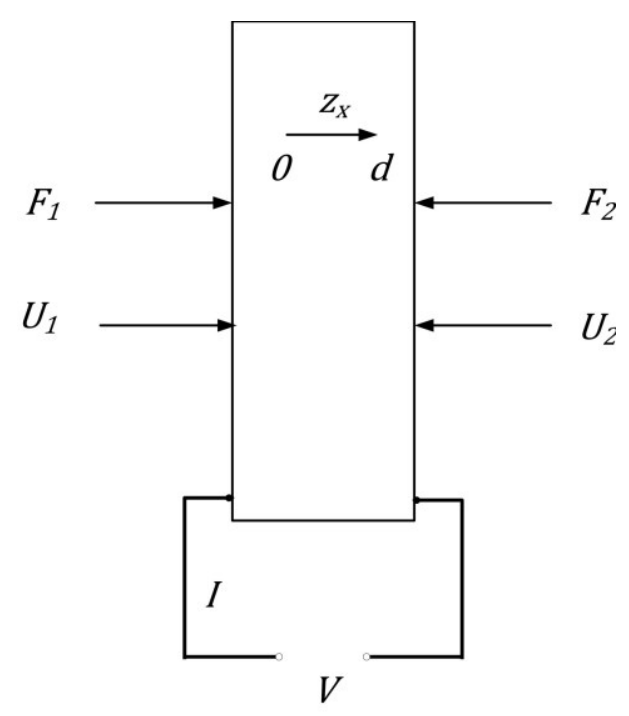

Figure 4.1: Three ports network of piezoelectric vibrates in thickness mode.

\subsubsection{Non-Piezoelectric Layer}

The ultrasonic sensor is constructed of a piezoelectric film layer and several nonpiezoelectric layers on the back and the front sides $\left(1,2, \ldots, \mathrm{n}\right.$ and $\left.1{ }^{\prime}, 2{ }^{\prime}, \ldots, \mathrm{n}^{\prime}\right)$ such as back and front electrodes, protection layer and backing material. The general configuration of an ultrasonic sensor is shown in Figure 4.2.

These non-piezoelectric layers in the ultrasonic sensor have no electric term. Thus, the relation in equation (4.4) yields for the non-piezoelectric $i$-th layer is expressed without the $V$ and $I$ as [57] 


$$
\left(\begin{array}{l}
F_{1 i} \\
U_{1 i}
\end{array}\right)=\left(\begin{array}{cc}
\cosh \gamma_{i} d_{i} & -j B_{i} \sinh \gamma_{i} d_{i} \\
-j / B_{i} \sinh \gamma_{i} d_{i} & \cosh \gamma_{i} d_{i}
\end{array}\right)\left(\begin{array}{l}
F_{2 i} \\
U_{2 i}
\end{array}\right)
$$

where $\delta_{m}=0$ and $\delta_{e}=0$ for the non-piezoelectric layers, $B_{i}$ and $\gamma_{i}$ are represented as:

$$
\begin{gathered}
B_{i}=j \rho_{i} v_{i} A, \\
\gamma_{i}=\frac{j \omega}{v_{i}},
\end{gathered}
$$

where $\rho_{i}, v_{i}$ and $d_{i}$ are the density, longitude sound velocity and thickness of the nonpiezoelectric layers, respectively.



Figure 4.2: General layers configuration of an ultrasonic sensor. 


\subsection{Simulation Model}

This section explains the equivalent electric circuit model of the developed sensor structure, which was an expansion of the Mason's model. The electromechanical expressions, including the dielectric and mechanical loss factors, that were discussed in the previous section have been introduced for an accurate simulation. Also, the derivation of the input impedance of the sensor is presented in this section to calculate the conversion efficiency of the sensor.

\subsubsection{Equivalent Circuit Model}

The equivalent circuit model of the developed sensor was derived based on the Mason circuit. The dielectric and mechanical losses were considered in the model by representing the piezoelectric coefficients with the real and complex components, as described in the Section 4.1.1. Figure 4.3 shows the Mason's equivalent circuit composed of a piezoelectric layer, back and front non-piezoelectric layers, and front and back mediums. . $Z_{f}$ and $Z_{b}$ are the acoustic impedance of front and back mediums, and [59]

$$
\begin{aligned}
& Z_{A i}=-j B_{i} \tanh \left(\frac{\gamma_{i} d_{i}}{2}\right), \\
& Z_{C i}=-j B_{i} \operatorname{csch}\left(\gamma_{i} d_{i}\right),
\end{aligned}
$$

where index $i=0,1,2, \ldots, \mathrm{n}, 1^{`}, 2^{`}, \ldots, \mathrm{n}^{`}$.

The sensor developed in this thesis consists of seven layers: PVDF film, front and back electrodes, polyimide film, and silicone adhesive, as shown in Figure 4.4. The

equivalent circuit model of the developed sensor is shown in Figure 4.5. The electric power source is connected to the PVDF film layer. The right side layers of the circuit are 
the front electrode, the front silicone adhesive, the front polyimide film, and the propagation medium. Similarly, the left side layers are the back electrode, the back silicone adhesive, the back polyimide film and the back medium. The electric power is converted into forward and backward acoustic power by the piezoelectric layer. The forward acoustic power propagates through the front layers to the propagation medium, while the backward power propagates through the back layers to the back medium.

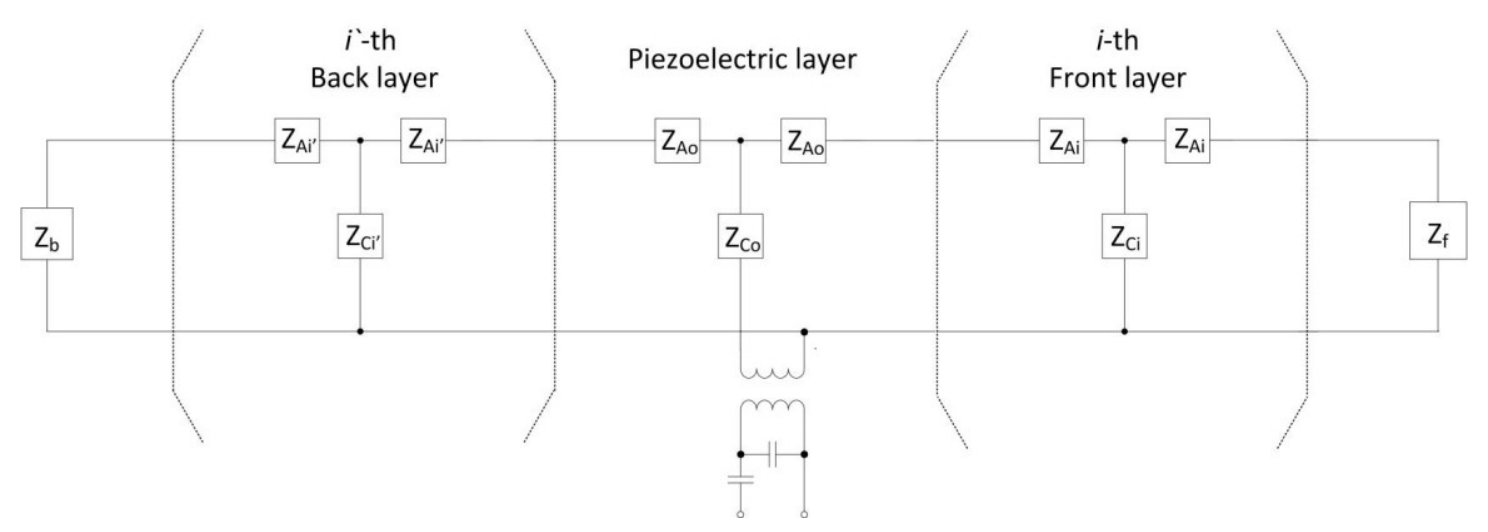

Figure 4.3: Mason's equivalent circuit.

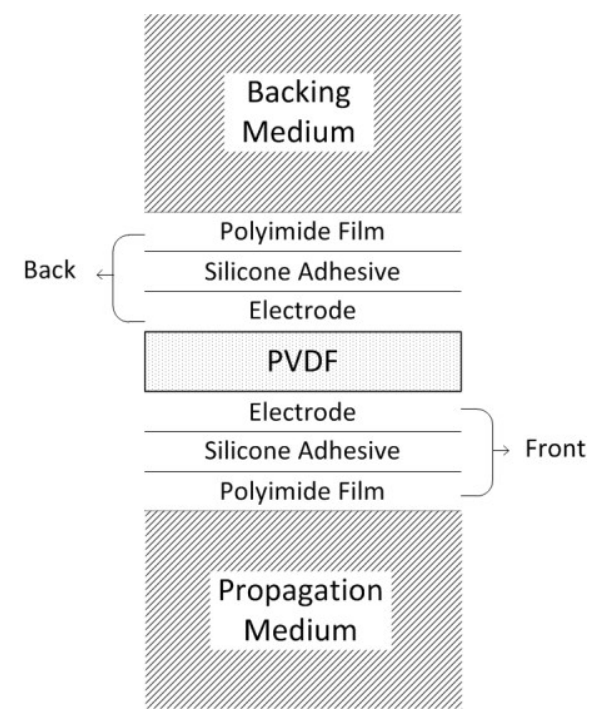

Figure 4.4: Developed sensor basic layers structure. 




\subsubsection{Sensor Impedance Derivation}

In order to derive the sensor impedance, $Z_{t}$, from the equivalent circuit, the impedances of the back layers (back electrode, back silicone adhesive, back polyimide film, and back medium) must first be simplified into one equivalent impedance, $Z_{B L}$, as shown in Figure 4.6.

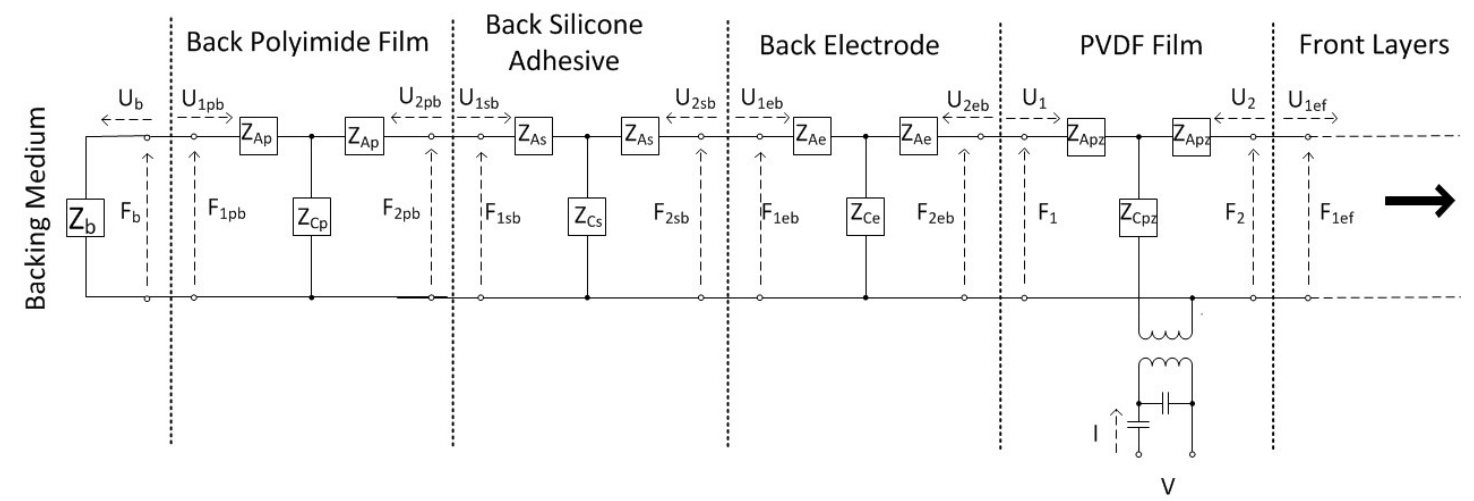

(a)

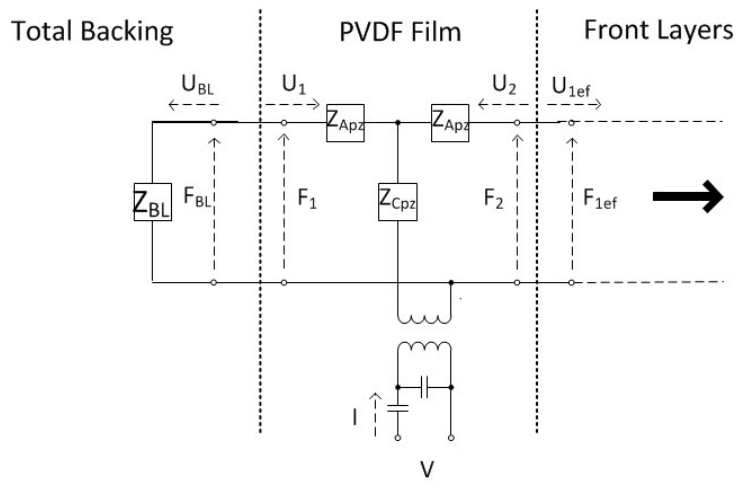

(b)

Figure 4.6: Developed sensor's equivalent circuit of the back side (a), and the equivalent back layers impedance, $Z_{B L},(b)$. 
The sensor impedance, $Z_{t}$, back layers impedance, $Z_{B k}$, back medium impedance, $Z_{b}$, and propagation medium impedance, $Z_{f}$, can be defined from Figure 4.5 as:

$$
\begin{gathered}
Z_{t}=\frac{V}{I} . \\
Z_{B L}=\frac{F_{2 e b}}{U_{2 e b}}, \\
Z_{b}=\frac{F_{b}}{U_{b}}=-\frac{F_{1 p b}}{U_{1 p b}}, \\
Z_{f}=\frac{F_{f}}{U_{f}}=-\frac{F_{2 p f}}{U_{2 p f}},
\end{gathered}
$$

where subscript $b$ represents the backing medium; $f$, the front (propagation) medium; $e b$, the back electrode layer; $p b$, the back polyimide layer; $p f$, the front polyimide layer. Subscript 1 and 2 in $V$ and $U$, represent the force and velocities at the front and the back side of each layer. Also, $F_{1 p b}$ and $U_{1 p b}$ of the back polyimide film can be expressed from equation (4.9) as:

$$
\left(\begin{array}{l}
F_{1 p b} \\
U_{1 p b}
\end{array}\right)=\left(\begin{array}{ll}
A_{p} & B_{p} \\
C_{p} & D_{p}
\end{array}\right)\left(\begin{array}{c}
F_{2 p b} \\
-U_{p b}
\end{array}\right)
$$

where $A, B, C$ and $D$ are:

$$
\left(\begin{array}{cc}
A_{i} & B_{i} \\
C_{i} & D_{i}
\end{array}\right)=\left(\begin{array}{cc}
\cosh \gamma_{i} d_{i} & -j B_{i} \sinh \gamma_{i} d_{i} \\
-j / B_{i} \sinh \gamma_{i} d_{i} & \cosh \gamma_{i} d_{i}
\end{array}\right)
$$

Thus, through all the back layers (back polyimide film, back silicone adhesive, and back electrode), $F_{1 p b}, U_{1 p b}$ could be expressed in terms of $F_{2 e b}$ and $U_{2 e b}$ as:

$$
\begin{gathered}
\left(\begin{array}{l}
F_{1 p b} \\
U_{1 p b}
\end{array}\right)=\left(\begin{array}{ll}
A_{p} & B_{p} \\
C_{p} & D_{p}
\end{array}\right)\left(\begin{array}{ll}
A_{s} & B_{s} \\
C_{s} & D_{s}
\end{array}\right)\left(\begin{array}{ll}
A_{e} & B_{e} \\
C_{e} & D_{e}
\end{array}\right)\left(\begin{array}{c}
F_{2 e b} \\
-U_{e b}
\end{array}\right) \\
=\left(\begin{array}{ll}
A_{B L} & B_{B L} \\
C_{B L} & D_{B L}
\end{array}\right)\left(\begin{array}{c}
F_{2 e b} \\
-U_{e b}
\end{array}\right),
\end{gathered}
$$


where subscript $p$ represents the polyimide layer; $s$, the silicone adhesive layer; $e$, the electrode layer. $A_{B L}, B_{B L}, C_{B L}$ and $D_{B L}$ are the multiplication of the back layer matrices. By inversing the equation, it yields:

$$
\begin{gathered}
\left(\begin{array}{c}
F_{2 e b} \\
U_{2 e b}
\end{array}\right)=\left(\begin{array}{ll}
A_{B L} & B_{B L} \\
C_{B L} & D_{B L}
\end{array}\right)^{-1}\left(\begin{array}{c}
F_{1 p b} \\
-U_{1 p b}
\end{array}\right) \\
=\left(\begin{array}{ll}
A^{\prime}{ }_{B L} & B^{\prime}{ }_{B L} \\
C^{\prime}{ }_{B L} & D^{\prime}{ }_{B L}
\end{array}\right)\left(\begin{array}{c}
F_{1 p b} \\
-U_{1 p b}
\end{array}\right) .
\end{gathered}
$$

Then, the back layers impedance $Z_{B L}$ is defined by combining equations (4.15), (4.16), and (4.21) as:

$$
Z_{B L}=\frac{A_{B L}{ }^{\prime} Z_{b}-B_{B L}{ }^{\prime}}{-C_{B L}{ }^{\prime} Z_{b}+D_{B L}{ }^{\prime}}
$$

$Z_{b}$ can be calculated by knowing the back medium density and the sound velocity as:

$$
Z_{b}=\rho_{b} v_{b} A .
$$

Consistent with deriving the sensor impedance, the linear relation between $V, F_{2}$ and $U_{2}$ at the PVDF film (piezoelectric layer) is converted into $2 \times 2$ matrix form by eliminating $F_{1}$ and $U_{1}$ from equation (4.4) and substituting equation (4.15). After some algebra, this yields an expression of:

$$
\left(\begin{array}{c}
V \\
I
\end{array}\right)=\left(\begin{array}{cc}
A_{p z} & B_{p z} \\
C_{p z} & D_{p z}
\end{array}\right)\left(\begin{array}{c}
F_{2} \\
-U_{2}
\end{array}\right),
$$

where $A_{p Z}, B_{p z}, C_{p z}$ and $D_{p z}$ are :

$$
\begin{gathered}
A_{p Z}=\frac{K_{1} K_{4}-K_{3}{ }^{2}+K_{4} Z_{B L}}{K_{3}\left(K_{1}-K_{2}+Z_{B L}\right)} \\
B_{p Z}=\frac{\left(K_{1} K_{4}-K_{3}{ }^{2}+K_{4} Z_{B L}\right)\left(K_{1}{ }^{2}-K_{2}{ }^{2}+K_{1} Z_{B L}\right)-K_{3}{ }^{2}\left(K_{1}-K_{2}+Z_{B L}\right)^{2}}{K_{3}\left(K_{1}-K_{2}+Z_{B L}\right)\left(Z_{B L}+K_{1}\right)}
\end{gathered}
$$




$$
\begin{gathered}
C_{p Z}=\frac{Z_{B L}+K_{1}}{K_{3}\left(K_{1}-K_{2}+Z_{B L}\right)}, \\
D_{p Z}=\frac{K_{1}{ }^{2}-K_{2}{ }^{2}+K_{1} Z_{B L}}{K_{3}\left(K_{1}-K_{2}+Z_{B L}\right)},
\end{gathered}
$$

where $K_{1}, K_{2}, K_{3}$ and $K_{4}$ are:

$$
\left(\begin{array}{lll}
K_{1} & K_{2} & K_{3} \\
K_{2} & K_{1} & K_{3} \\
K_{3} & K_{3} & K_{4}
\end{array}\right)=\left(\begin{array}{ccc}
-j B \operatorname{coth} \gamma d & -j B \operatorname{csch} \gamma d & -j h / \omega \\
-j B \operatorname{csch} \gamma d & -j B \operatorname{coth} \gamma d & -j h / \omega \\
-j h / \omega & -j h / \omega & -j 1 / C \omega
\end{array}\right) .
$$

From equation (4.24), we can define $V$ and $I$ in terms of $F_{f}$ and $U_{f}$, by including the entire front layer (front electrode, front silicone adhesive, and front polyimide film) matrices as:

$$
\left(\begin{array}{l}
V \\
I
\end{array}\right)=\left(\begin{array}{ll}
A_{p z} & B_{p z} \\
C_{p z} & D_{p z}
\end{array}\right)\left(\begin{array}{cc}
A_{e} & B_{e} \\
C_{e} & D_{e}
\end{array}\right)\left(\begin{array}{ll}
A_{s} & B_{s} \\
C_{s} & D_{s}
\end{array}\right)\left(\begin{array}{ll}
A_{p} & B_{p} \\
C_{p} & D_{p}
\end{array}\right)\left(\begin{array}{l}
F_{f} \\
U_{f}
\end{array}\right) .
$$

Multiplying the matrices yields:

$$
\left(\begin{array}{l}
V \\
I
\end{array}\right)=\left(\begin{array}{ll}
A_{F L} & B_{F L} \\
C_{F L} & D_{F L}
\end{array}\right)\left(\begin{array}{l}
F_{f} \\
U_{f}
\end{array}\right)
$$

where $A_{F L}, B_{F L}, C_{F L}$ and $D_{F L}$ are the multiplication of the front layer matrices. Thus, substituting (4.17) in (4.31) brings the expression of the sensor impedance $Z_{t}$ as:

$$
Z_{t}=\frac{V}{I}=\frac{A_{F L} Z_{f}+B_{F L}}{C_{F L} Z_{f}+D_{F L}} .
$$

$Z_{f}$ can be calculated by knowing the propagation medium density and sound velocity as:

$$
Z_{f}=\rho_{f} v_{f} A .
$$




\subsubsection{Conversion Efficiency}

In this simulation model, we calculate the matching loss (ML), conversion loss (CL), and transducer loss (TL) to evaluate the performance of the developed sensor. These parameters are defined as:[57], [59]

$$
\begin{gathered}
M L=-10 \log \frac{P_{t}}{P_{o}}=-10 \log \frac{4 \operatorname{Re}\left(Z_{s}\right) \operatorname{Re}\left(Z_{t}\right)}{\left|Z_{s}+Z_{t}\right|^{2}}, \\
C L=-10 \log \frac{P_{m f}}{P_{t}}=-10 \log \frac{\operatorname{Re}\left(Z_{f}\right)\left|Z_{t}\right|^{2}}{\left|A_{F t} Z_{f}+B_{F t}\right|^{2} \operatorname{Re}\left(Z_{t}\right)}, \\
T L=M L+C L,
\end{gathered}
$$

where $Z_{s}$ is the impedance of the electric source that provides electric power to the sensor, and $R e$ represents the real part of the complex component. The matching loss (ML) represents the ratio between the power transmitted into the sensor $P_{t}$ and the maximum power available from the electric source $P_{o}$. The difference between $P_{o}$ and $P_{t}$ represents the reflected power back from the sensor to the source, due to the impedance mismatch between the source and the sensor. The conversion loss (CL) is the conversion efficiency of the electric power into the acoustic power. The power transmitted into the sensor $P_{t}$ is converted into forward acoustic power $P_{m f}$ (on the propagation medium side), backward acoustic power $P_{m b}$ and thermal dissipating power $P_{l}$. Combining matching loss (ML) and conversion (CL) gives the ratio of acoustic power output of the sensor to the power driven from the electric source, which is described as the transducer loss (TL). 


\section{Chapter 5: Ultrasonic Sensor Characterization}

The following chapter deals with the evaluation of the performance and behavior

of the developed sensor. It provides a detailed study and discussion about the frequency characteristics and electromechanical activity for different sensor based on the numerical calculations of the sensor simulation model, given in chapter 4 , and the experimental results. In addition, we investigate several ultrasonic couplants and discuss their performance for the proposed application.

\subsection{Numerical Calculation of Sensor Characteristics}

In this section, the frequency characteristics of the developed sensor such as center frequency and bandwidth are investigated theoretically. In order to understand the sensor frequency behaviour, the effects of the non-piezoelectric layers of the electrodes and of the protection/isolation layer on the PVDF frequency resonance were first assessed independently then combined together. The acoustic properties of the materials used in the numerical calculations are listed in Table 5.1. 
Table 5.1: Acoustic properties of the materials used in the numerical calculations.

\begin{tabular}{|c|c|c|c|}
\hline Material & $\begin{array}{c}\text { Density } \\
{\left[\times 10^{3} \mathrm{~kg} / \mathrm{m}^{3}\right]}\end{array}$ & $\begin{array}{l}\text { Sound Velocity } \\
{[\mathrm{m} / \mathrm{s}]}\end{array}$ & $\begin{array}{l}\text { Acoustic Impedance } \\
{\left[\times 10^{6} \mathrm{~kg} /\left(\mathrm{m}^{2} \mathrm{~s}\right)\right]}\end{array}$ \\
\hline Air $(20 C)^{a}$ & $1.24 \times 10^{-3}$ & 344 & $427 \mathrm{~kg} /\left(\mathrm{m} \mathrm{s}^{2}\right)$ \\
\hline Water (at 20C) & 1.0 & 1480 & 1.48 \\
\hline $\mathrm{PVDF}^{\mathrm{c}}$ & 1.78 & 2260 & 4.02 \\
\hline Cupper $(\mathrm{Cu})^{\mathrm{b}}$ & 8.93 & 5010 & 44.6 \\
\hline Nickel (Ni) ${ }^{b}$ & 8.84 & 5600 & 49.5 \\
\hline Steel $^{b}$ & 7.89 & 5790 & 45.7 \\
\hline Silver $(A g)^{b}$ & 10.6 & 3600 & 38 \\
\hline Silver epoxy ${ }^{b}$ & 2.71 & 1900 & 5.14 \\
\hline Silver paint ${ }^{\mathrm{c}}$ & 3.4 & 3600 & 12.24 \\
\hline Silicone adhesive & 1.47 & 960 & 1.41 \\
\hline Polyimide film ${ }^{\mathrm{d}}$ & 1.421 & 2414 & 3.4 \\
\hline Plexiglas (PMMA) $^{b}$ & 1.19 & 2750 & 3.27 \\
\hline
\end{tabular}

${ }^{\mathrm{a}}$ from [60]. ${ }^{\mathrm{b}}$ from [64]. ${ }^{\mathrm{c}}$ estimated. ${ }^{\mathrm{d}}$ from [65]. 


\subsubsection{PVDF Resonance Frequency}

The ultrasonic sensor composed of piezoelectric polymer PVDF without backing layers operates in the $(2 n-1) \lambda / 2$ thickness mode, where $\lambda$ is the acoustic wave length in the PVDF, and $n=1,2,3, \ldots$. . The resonance frequency of a $110-\mu \mathrm{m}$ thick PVDF layer, is shown in Figure 5.1(a), has been calculated based on the simulation model and the equations described in Chapter 4. The propagation medium was assumed to be water, and the backing medium to be air. The impedance of an electric power source set to $50 \Omega$, and the size of the sensor was $1.5 \mathrm{~cm} \times 1.5 \mathrm{~cm}$.

Figure 5.1(b) shows the calculated transducer loss (TL) of the sensor configuration given in Figure 5.1(a). The fundamental resonance frequency $f_{o}$ of the 110$\mu \mathrm{m}$ thick PVDF film is the frequency with the minimum loss at $10 \mathrm{MHz}$. Also, from the sensor impedance calculated in Figure 5.2, the largest sensor impedance magnitude transition and phase shift occurred at $10 \mathrm{MHz}$ representing the first resonant frequency. The smaller phase shifts at $30 \mathrm{MHz}$ and at $50 \mathrm{MHz}$ in Figure 5.2 (b) represents the second and the third resonant frequencies. 


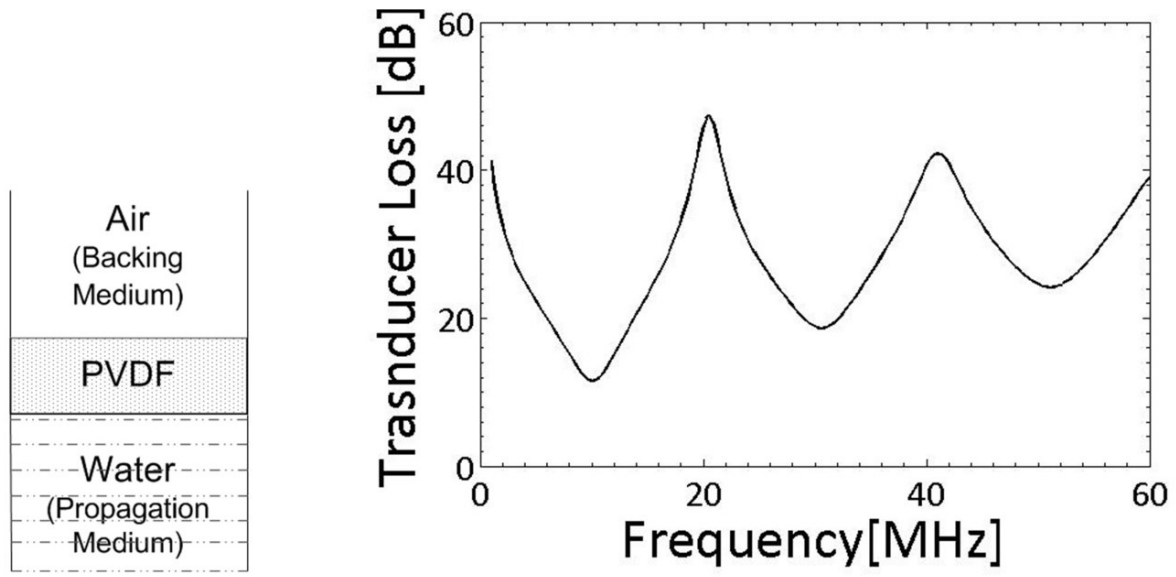

(a)

(b)

Figure 5.1: Frequency response of ultrasonic sensor composed of PVDF film only; (a) sensor structure, (b) transducer loss (TL). 


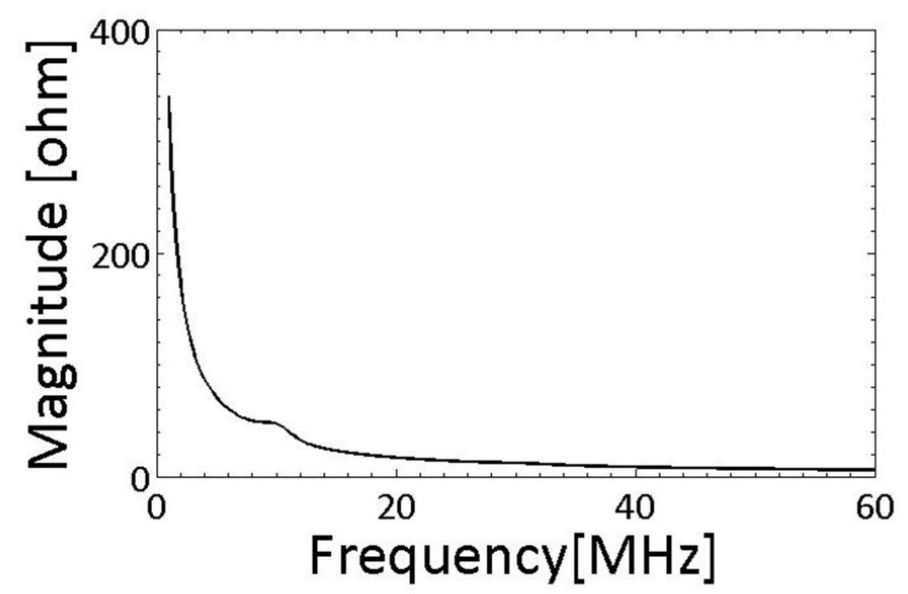

(a)

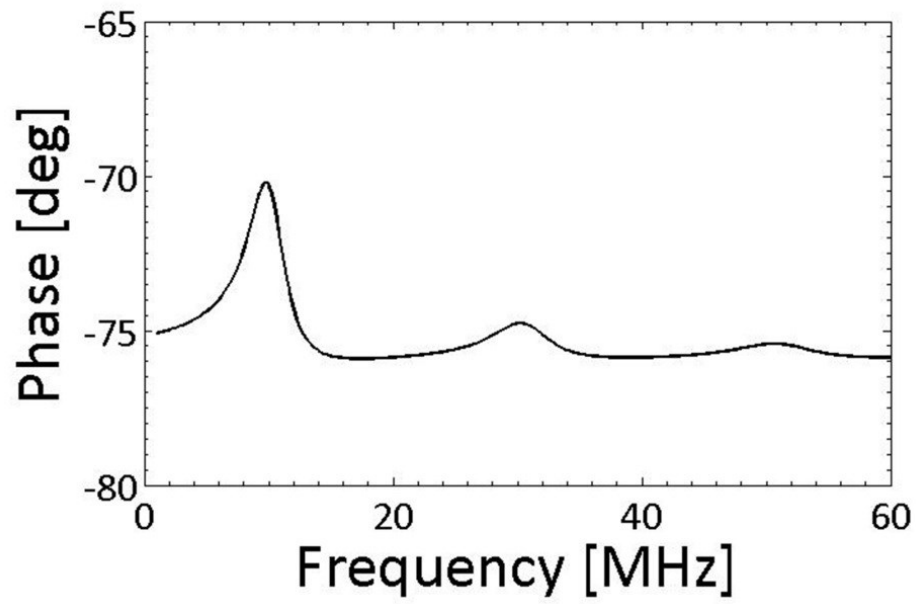

(b)

Figure 5.2: Frequency response of ultrasonic sensor composed of PVDF film only; (a) impedance magnitude, (b) impedance phase. 
Figure 5.3 shows the calculated transducer loss (TL), conversion loss (CL) and matching loss (ML) for the same sensor configuration illustrated in Figure 5.1(a), in two different cases: one with internal losses included and the other without losses. In both cases, the piezoelectric constants were assumed to be the PVDF film properties listed in Table 3.1 (chapter 3 ), except the mechanical loss factor tan $\delta_{m}$ and the dielectric loss factor $\tan \delta_{e}$, which were zero for the case without internal losses. In Figure 5.3(a), both cases have a similar transducer loss (TL) behavior, in terms of the resonance frequency, with a minimum loss value at $10 \mathrm{MHz}$. However, the bandwidths of the resonances frequency with the internal losses became broader, and the transducer loss increased by $3.54 \mathrm{~dB}$ at $10 \mathrm{MHz}$. Contrary to the transducer loss (TL), conversion loss (CL) in Figure 5.3(b) significantly increased when the internal losses were included, representing the amount of thermal dissipation resulting from the electromechanical conversion at the piezoelectric polymer PVDF. In contrast, the matching loss (ML) on Figure 5.3(c) is greatly decreased with internal losses implying that the dielectric loss factor $\tan \delta_{e}$ improves the impedance mismatch between the sensor and the electric power source. Given these points, the internal losses on the piezoelectric polymer sensor help broaden the frequency bandwidth and enhance the impedance matching whereas they degrade the efficiency of the sensor's electrical to mechanical conversion [57]. 
(a)

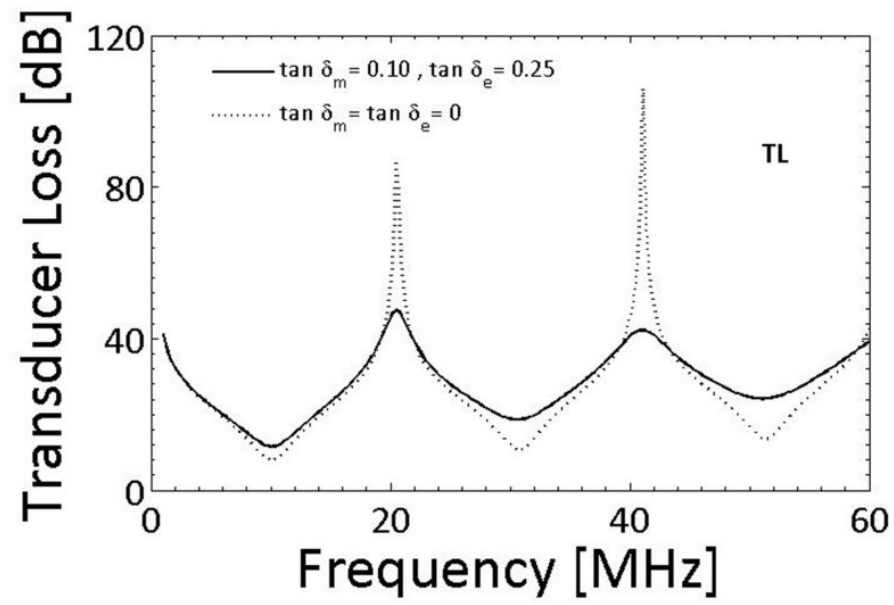

(b)

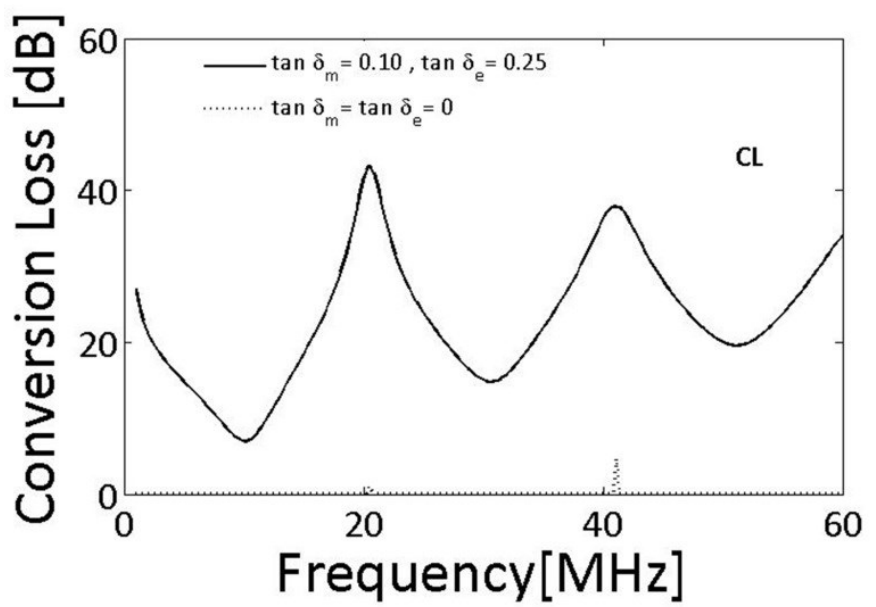

(c)

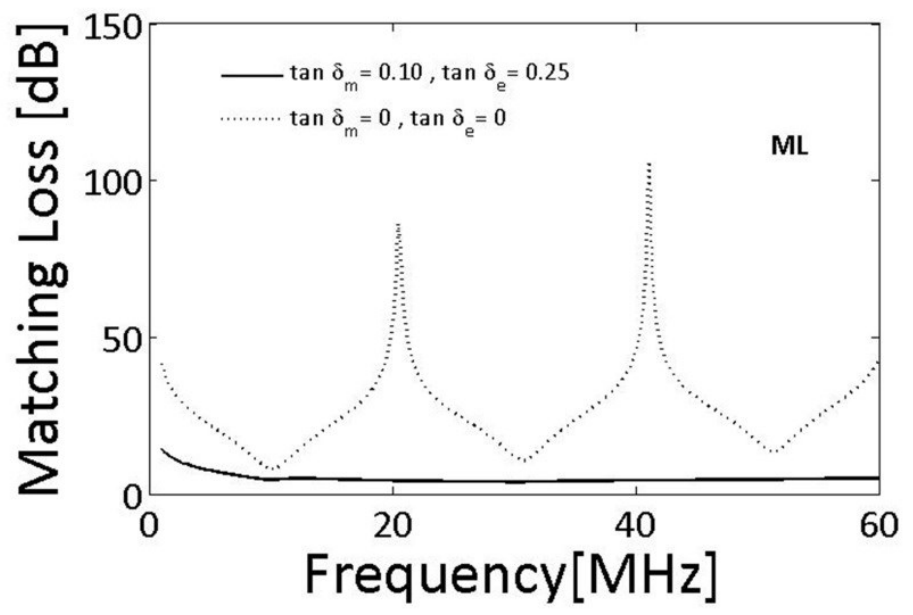

Figure 5.3: Frequency response of ultrasonic sensor showing the effect of the internal losses; (a) transducer loss, (b) conversion loss, (c) matching loss. 


\subsubsection{Electrode Layer Effect}

The frequency response of the PVDF film without non-piezoelectric layers was discussed in section 5.1.1. This section discusses the effect of the electrode layers on the frequency response of PVDF film.

Figure 5.4 presents the numerical simulation results of a $1.5 \mathrm{~cm} \times 1.5 \mathrm{~cm}$ sensor composed of PVDF for the piezoelectric layer and silver paint for the front and back electrode layers. The PVDF thickness is fixed at $110 \mu \mathrm{m}$ while the electrodes thickness varies from $0 \mu \mathrm{m}$ to $110 \mu \mathrm{m}$. The sensor is assumed to be air backed and operates in a water medium as illustrated in Figure 5.4(a). Figure 5.4(b) shows the effect of the electrode layers on the sensor's frequency response. When the front and back electrodes thickness changes from $0 \mu \mathrm{m}$ to $75 \mu \mathrm{m}$, the resonance frequency $f_{o}$ changes from $10 \mathrm{MHz}$ $\left(\lambda / 2\right.$ frequency) to $5 \mathrm{MHz}\left(\lambda / 4\right.$ frequency, $\left.0.5 f_{o}\right)$. In this thesis research, ultrasonic sensors were developed with two kinds of electrode materials, one is a $13-\mu \mathrm{m}$ thick silver paint, and the other is a $0.035-\mu \mathrm{m}$ thick copper-nickel composite, for each front and back layers as mentioned in Chapter 3. From Figure 5.4(b), it can be seen that the 26- $\mu \mathrm{m}$ thick silver paint electrode layers lowered the resonance frequency to $7 \mathrm{MHZ}$ whereas the electrode layers with a thickness of less than $1 \mu \mathrm{m}$ have a negligible effect (less than $2 \%$ ) on the frequency response. Furthermore, Figure 5.5 compares the loss parameters of the PVDF frequency response with the $26-\mu \mathrm{m}$ thick silver paint $(13 \mu \mathrm{m}$ for each of the front and the back sides $)$ and the $0.07-\mu \mathrm{m}$ copper-nickel $(0.035 \mu \mathrm{m}$ for each of the front and the back sides) electrode layers. They were calculated with the configuration in Figure 5.4. The 0.07- $\mu \mathrm{m}$ copper-nickel electrode shows no significant impact on the frequency response of the PVDF sensor. Therefore, the mass-loading of the copper-nickel 
electrode could be considered negligible. From Figure 5.5(a), it is observed that the silver paint electrode increased the matching loss at the fundamental resonance frequency by $0.8 \mathrm{~dB}$. On the contrary, it improved the conversion loss by $0.6 \mathrm{~dB}$ as seen from Figure 5.5(b). As a result, the transducer remained constant, as shown in Figure 5.5(c). However, the resonance frequency was shifted to a lower range and became slightly narrower. Thus, the $26-\mu \mathrm{m}$ thick silver paint electrode layers affect the sensor's characteristics by decreasing the resonance frequencies and narrowing the frequency bandwidth. It improves the sensor's conversion efficiency whereas it increases the electrical impedance mismatching. Generally, metal electrodes have greater acoustic impedance $\left(12.24 \times 10^{6} \mathrm{~kg} / \mathrm{m}^{2} \mathrm{~s}\right.$ for silver paint) than the piezoelectric polymer PVDF $\left(4.02 \times 10^{6} \mathrm{~kg} / \mathrm{m}^{2} \mathrm{~s}\right)$, as shown Table 5.1, which affects the frequency response of the PVDF sensor by shifting the resonance frequencies to a lower range. Also, the thicker metal electrode layers enlarge the effect due to their large mass-loading on the piezoelectric film. 


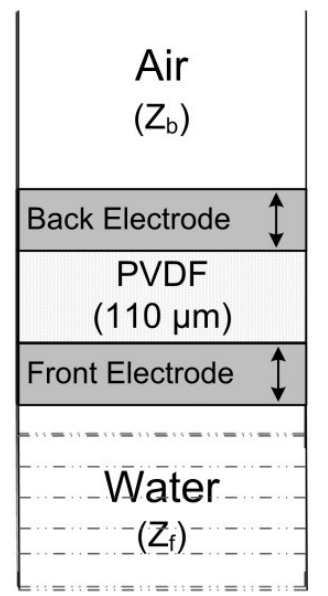

(a)

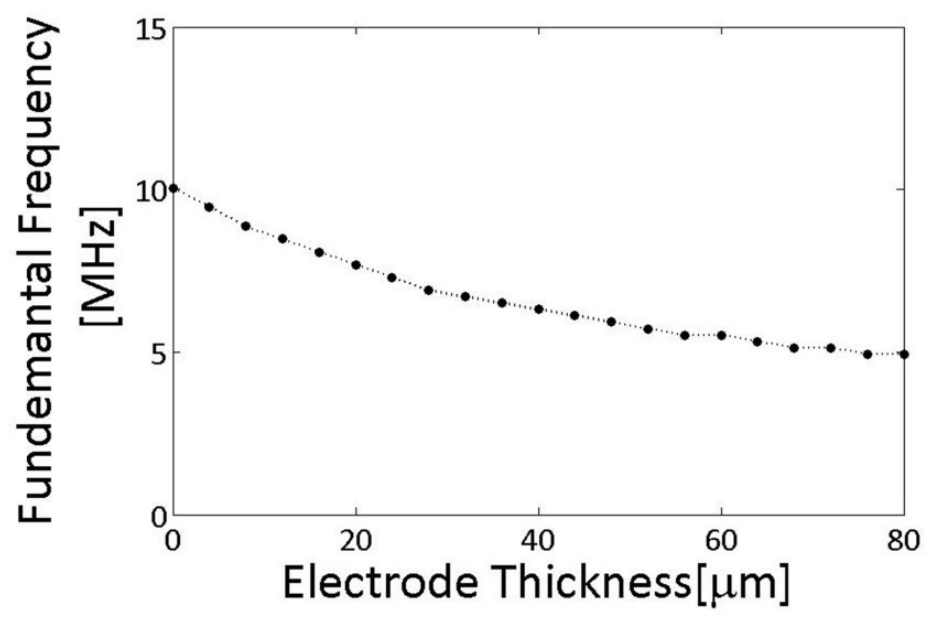

(b)

Figure 5.4: Numerical calculation results of the fundamental resonant frequency for the PVDF film as a function of front and back silver paint layer thickness; (a) sensor structure, (b) fundamental resonant frequency. 
(a)

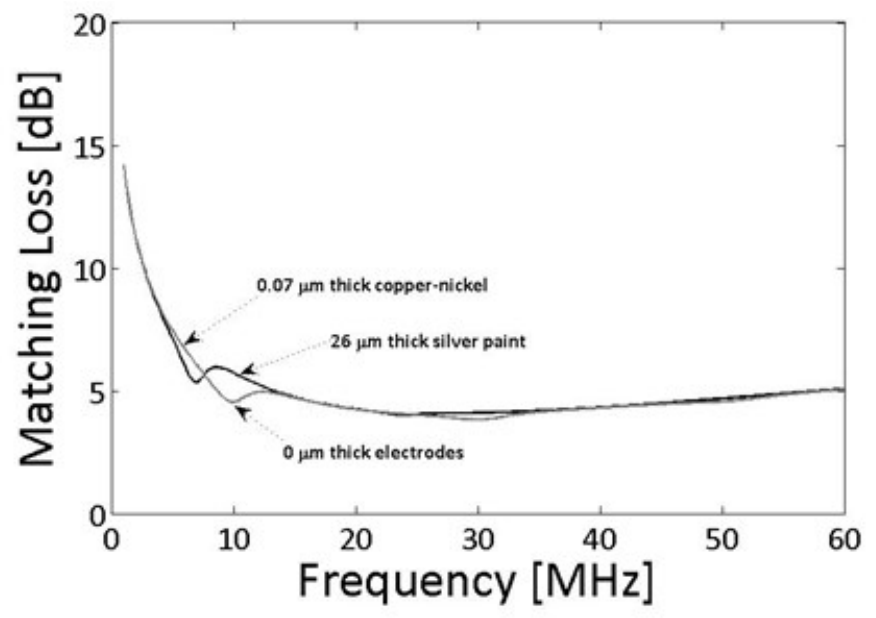

(b)

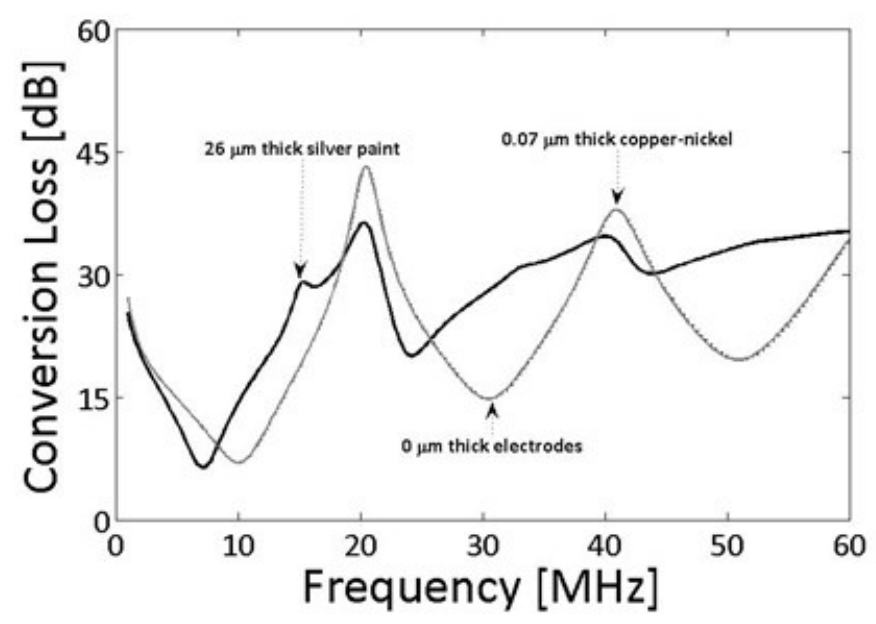

(c)

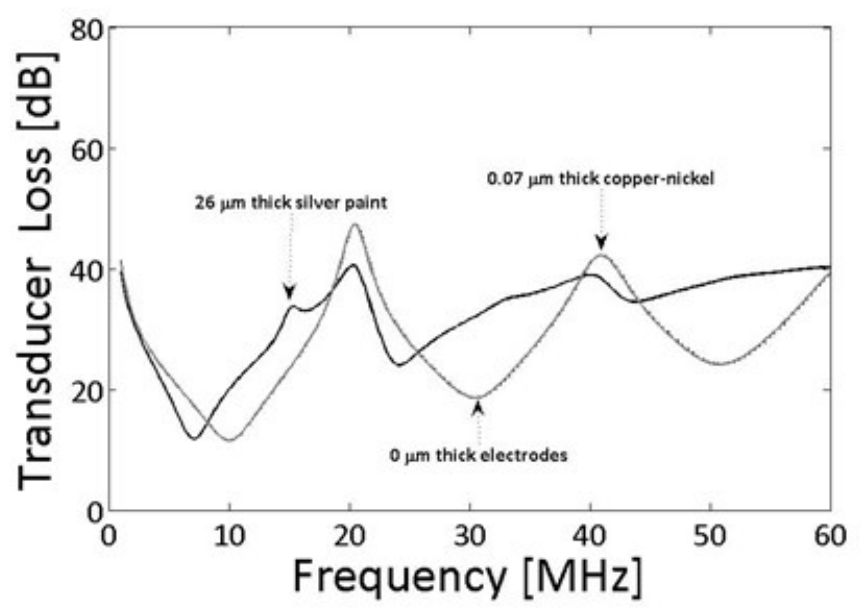

Figure 5.5: comparing the effect of silver paint and copper-nickel (top and bottom electrodes) to the PVDF resonance frequency; (a) matching loss (ML), (b) conversion 


\subsubsection{Protection/Isolation Layer Effect}

The protection/isolation layer is composed of a silicone adhesive and a polyimide film. In order to investigate the effect of the protection layer on the sensor frequency characteristics, the silicone adhesive and the polyimide film were first investigated independently.

Air has a very low acoustic impedance of $427 \mathrm{~kg} / \mathrm{m}^{2} \mathrm{~s}\left(10^{8}\right.$ times smaller than PVDF) which has a negligible effect on the sensor performance. However, silicone adhesive $\left(1.44 \times 10^{6} \mathrm{~kg} / \mathrm{m}^{2} \mathrm{~s}\right)$ has a closer acoustic impedance to the PVDF $\left(4.02 \times 10^{6} \mathrm{~kg} / \mathrm{m}^{2} \mathrm{~s}\right)$, and its effect largely depends on the thickness of the silicone layers. Figure 5.6(b) shows the calculated transducer loss (TL) of a 110- $\mu \mathrm{m}$ thick PVDF with front and back silicone adhesive layers, with total thickness ranging from $0 \mu \mathrm{m}$ to 110 $\mu \mathrm{m}$. The propagation medium and back medium are assumed to be water and air, respectively, as shown in Figure 5.6(a). The source impedance is set to be $50 \Omega$. Figure 5.6(b) shows that when the total thickness of the front and back silicone adhesive layers is between $1 \mu \mathrm{m}$ and $20 \mu \mathrm{m}$, there is no significant effect on the fundamental resonance frequency of the PVDF film (around $10 \mathrm{MHz}$ where the TL is the minimum). When the thickness increases from $20 \mu \mathrm{m}$ to $40 \mu \mathrm{m}$, the resonance frequency starts shifting to the lower frequency, around $8.7 \mathrm{MHz}$. with a total thickness of $50 \mu \mathrm{m}$, the sensor has two narrow center frequencies of 7.9 and $11.8 \mathrm{MHz}$. These two center frequencies keep shifting to lower frequencies as the thickness increases. 


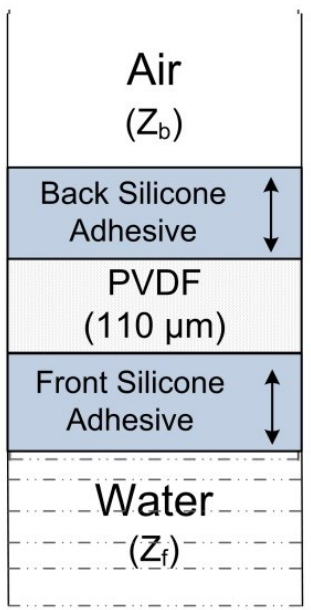

(a)

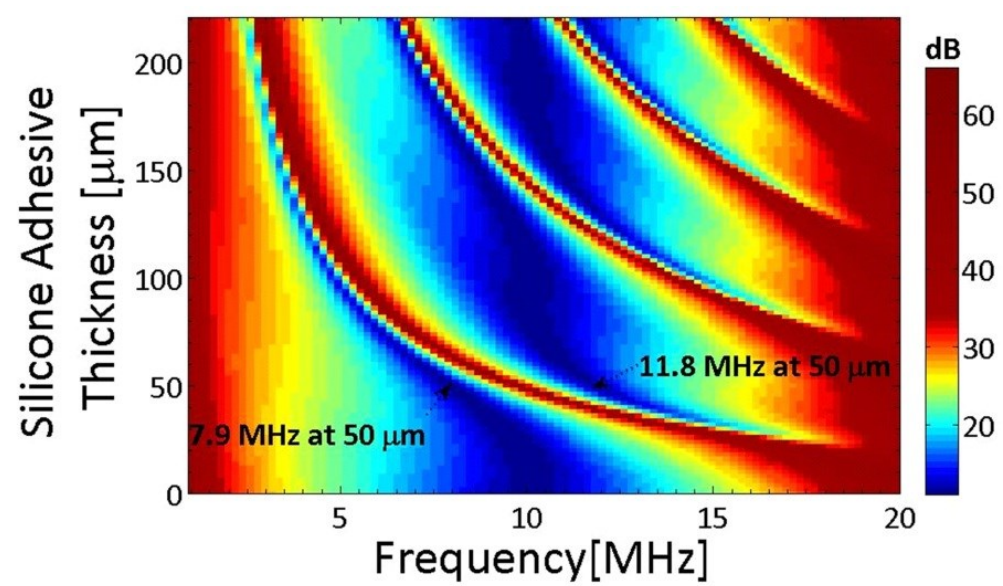

(b)

Figure 5.6: transducer loss of ultrasonic sensor composed of 110- $\mu \mathrm{m}$ thick PVDF film with front and back silicone adhesive; (a) sensor structure, (b) transducer loss (TL).

In the structure of the developed sensor, the polyimide protection layers were glued to the top and the bottom electrodes using silicone adhesive layer of $25-\mu \mathrm{m}$ thick. The sensor's performance with this thickness of silicone adhesive layers was calculated and is shown in Figure 5.7. Note that the polyimide film thickness was assumed to be zero in this calculation. The structure and the loss parameters of the $110-\mu \mathrm{m}$ thick PVDF sensor with $25-\mu \mathrm{m}$ thick silicone adhesive layers are illustrated in Figure 5.7(a) and (b), respectively. With the front and back silicone adhesive layers at a total thickness of 50$\mu \mathrm{m}$, the sensor still resonates at $10 \mathrm{MHz}$ but the reflection at the silicone/air interface causes a destructive interference, resulting in a large loss at the resonance frequency. For this reason, the PVDF film with a $50-\mu \mathrm{m}$ silicone adhesive operates at two narrow center frequencies at 7.9 MHz and 11.8 MHz. At the center frequency of 7.9 MHz, the sensor has $11.8 \mathrm{~dB}$ TL, 6.9 dB CL and 4.7 dB ML while at the center frequency of $11.8 \mathrm{MHz}$ it has the same TL but a higher CL and a lower ML by $1 \mathrm{~dB}$. 




(a)

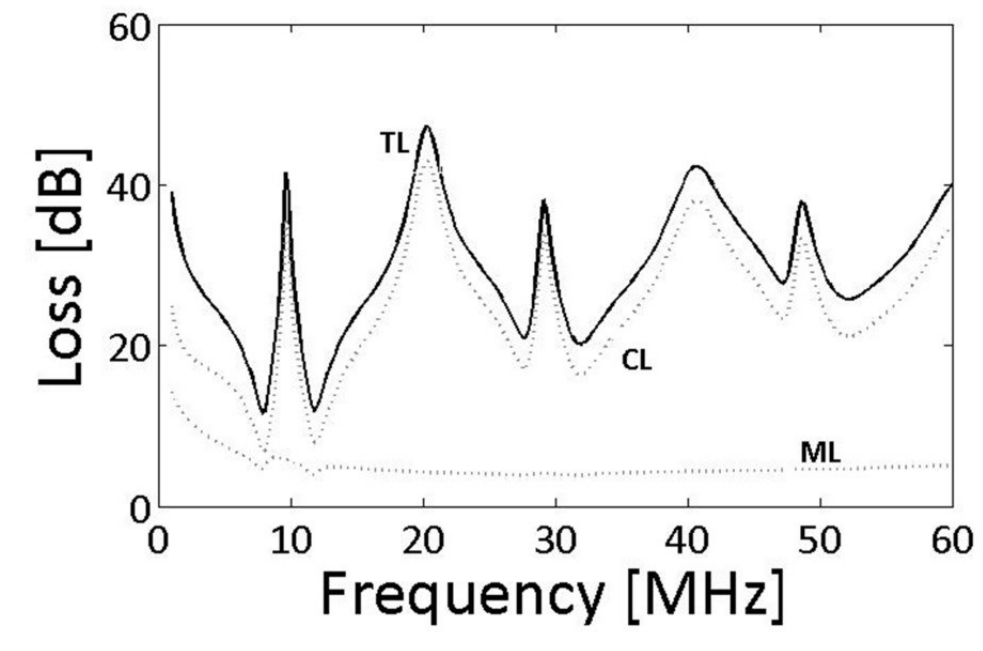

(b)

Figure 5.7: Calculated loss parameters of ultrasonic sensor composed of 110- $\mu \mathrm{m}$ thick

PVDF film and $25-\mu \mathrm{m}$ thick front and back silicone adhesive; (a) sensor structure, (b) losses (TL, CL, ML).

Moreover, the effect of the polyimide layer on the PVDF sensor without the silicone adhesive was also investigated. Figure 5.8 shows the transducer loss of the PVDF sensor having a front and a back polyimide layers with a total thickness ranging from $1 \mu \mathrm{m}$ to $220 \mu \mathrm{m}$. From Figure 5.8(b), it is observed that the sensor resonance frequency shifts to a lower range as the polyimide layers thickness increases.

Figure 5.9 shows the loss parameters of the PVDF sensor with $13-\mu \mathrm{m}$ thick layer of the front and the back polyimide film. Figure 5.9(a) illustrates the structure of the sensor with propagation and back media. The effect of the polyimide film layers is similar to the effect of the silver paint electrode layers. They increase the matching loss and improve the conversion loss as seen in Figure 5.9(b). The resonance frequency was shifted from $10 \mathrm{MHz}$ (the resonating frequency of a $110-\mu \mathrm{m}$ PVDF film) to $8.6 \mathrm{MHz}$ with same transducer loss of $11.6 \mathrm{~dB}$ due to the polyimide mass-loading. Also, the close 
matching of the polyimide acoustic impedance $\left(3.4 \times 10^{6} \mathrm{~kg} / \mathrm{m}^{2} \mathrm{~s}\right)$ to the PVDF acoustic impedance allows a broader resonance frequency of the PVDF sensor.

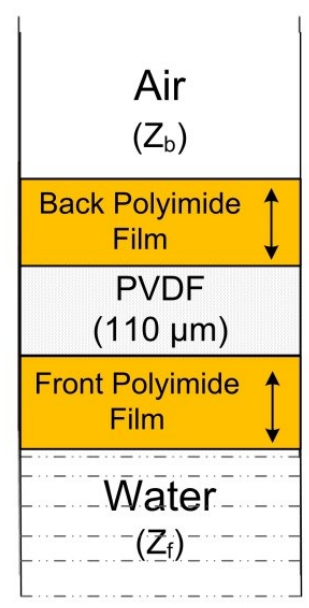

(a)

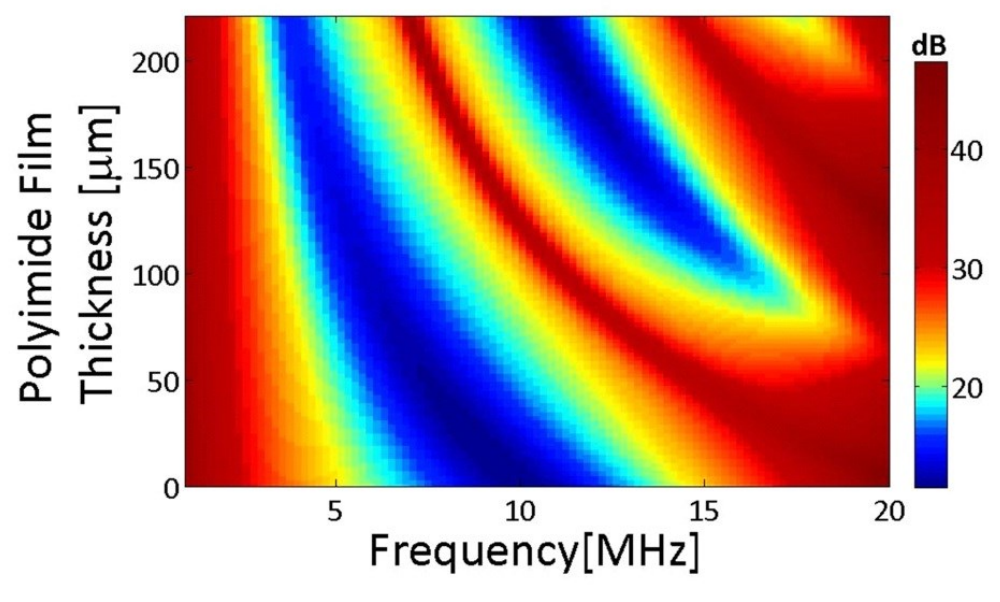

(b)

Figure 5.8: The transducer loss of ultrasonic sensor composed of 110- $\mu \mathrm{m}$ thick PVDF film with Polyimide layers in both sides; (a) sensor structure, (b) transducer loss (TL).

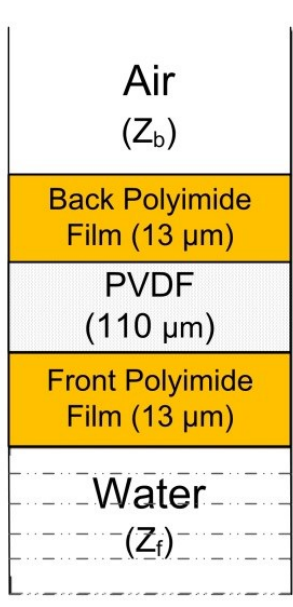

(a)

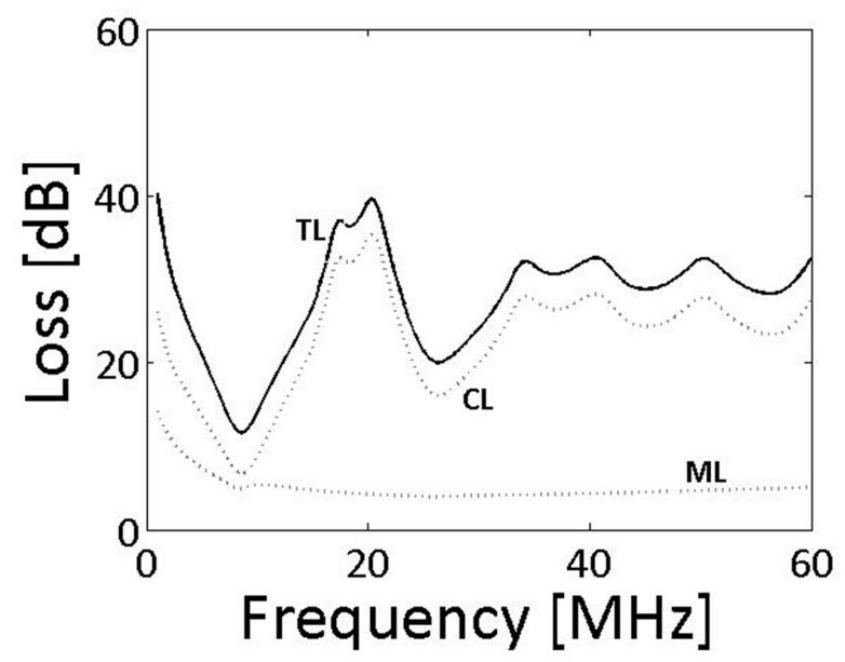

(b)

Figure 5.9: The loss parameters of ultrasonic sensor composed of $110-\mu \mathrm{m}$ thick PVDF

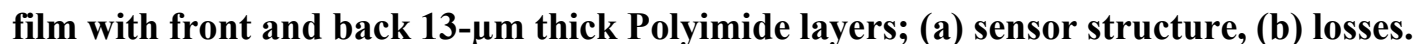


The effect of the silicone adhesive and the polyimide film together on the PVDF sensor is now investigated. Figure 5.10 shows the three structures of the PVDF sensor: (sp) both the $50-\mu \mathrm{m}$ silicone adhesive layers and the $26-\mu \mathrm{m}$ polyimide protection layers; (s) only the $50-\mu \mathrm{m}$ silicone adhesive layers; (p) only the $26-\mu \mathrm{m}$ polyimide protection layers. The numerical calculation results of all the structures have been plotted together to illustrate the differences, as seen in Figure 5.11.

From Figure 5.11(a), it is observed that the calculated loss parameters of the PVDF sensor composed of silicone adhesive and polyimide protection layers (structure "sp") has a slightly larger conversion loss of $7.5 \mathrm{~dB}$ at $5.8 \mathrm{MHz}$ and of $7.9 \mathrm{~dB}$ at 10.7 MHz compared to the structure of "s" and "p". On other hand, the PVDF sensor "sp" has a matching loss of $3.6 \mathrm{~dB}$ at $10.7 \mathrm{MHz}$, which is less than the structures "s" and "p" as seen in Figure 5.11(b). The sensor "sp" still resonates at $8.6 \mathrm{MHz}$ due to mass-loading of the polyimide layers, but the silicone adhesive causes a large loss at the resonance frequency (as discussed earlier). Therefore, the sensor "sp" operates at dual center frequencies at $5.8 \mathrm{MHz}$ and $10.7 \mathrm{MHz}$ as seen in Figure 5.11(c). 


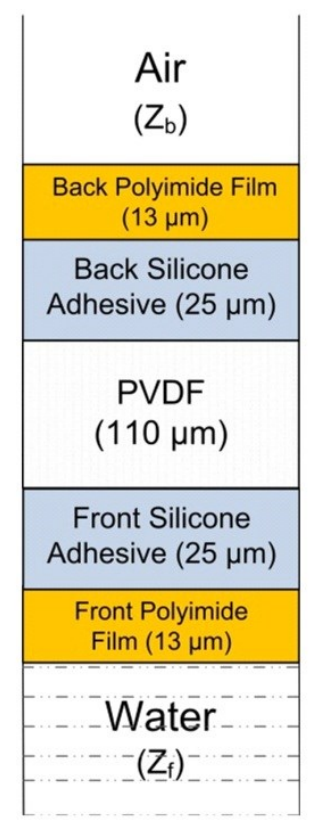

$(\mathrm{sp})$

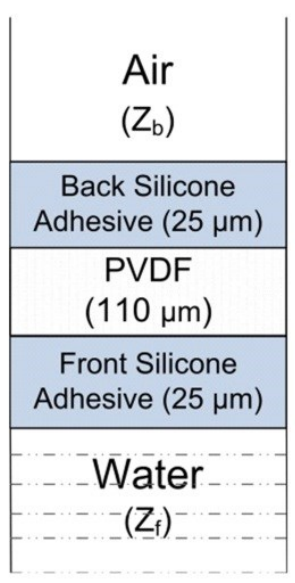

(s)

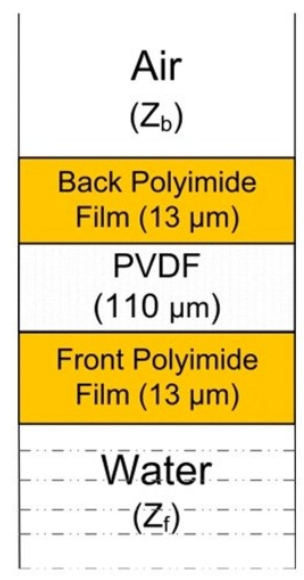

(p)

Figure 5.10: Structures of the three PVDF sensors compost; (sp) silicone adhesive and polyimide film layers, (s) silicone adhesive layers, (p) polyimide film layers (p). 
(a)

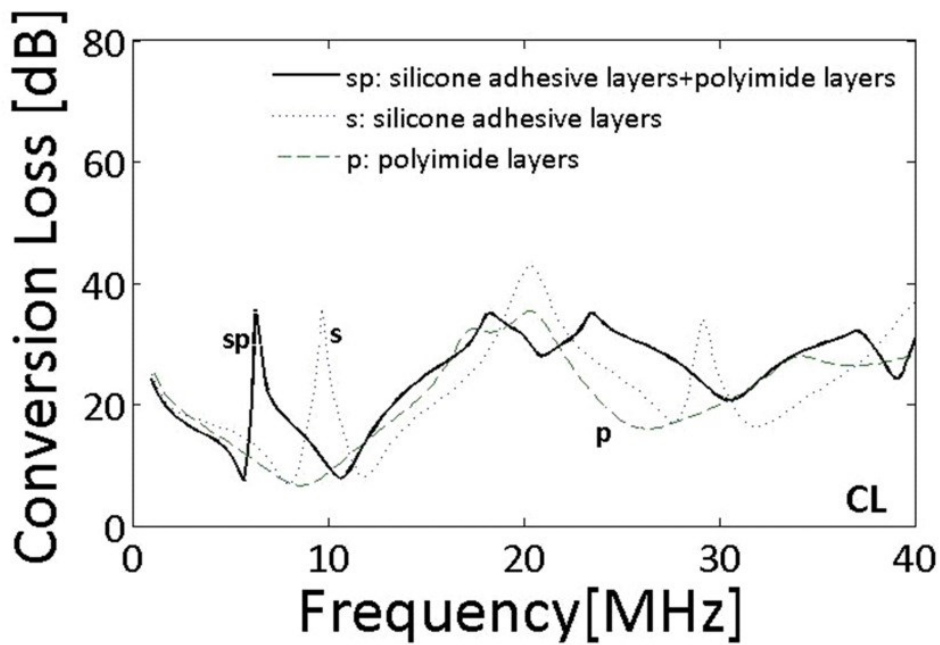

(b)

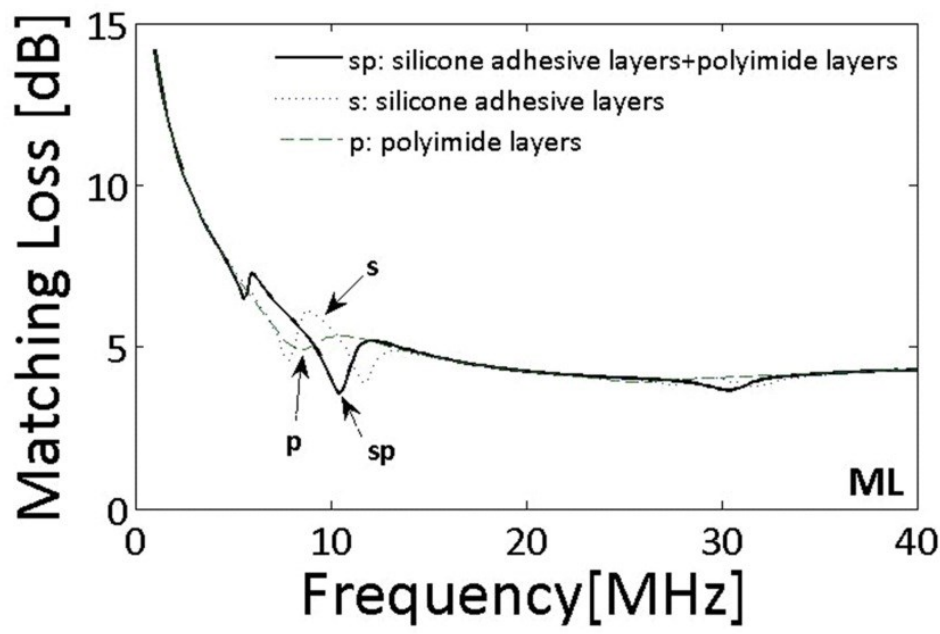

(c)

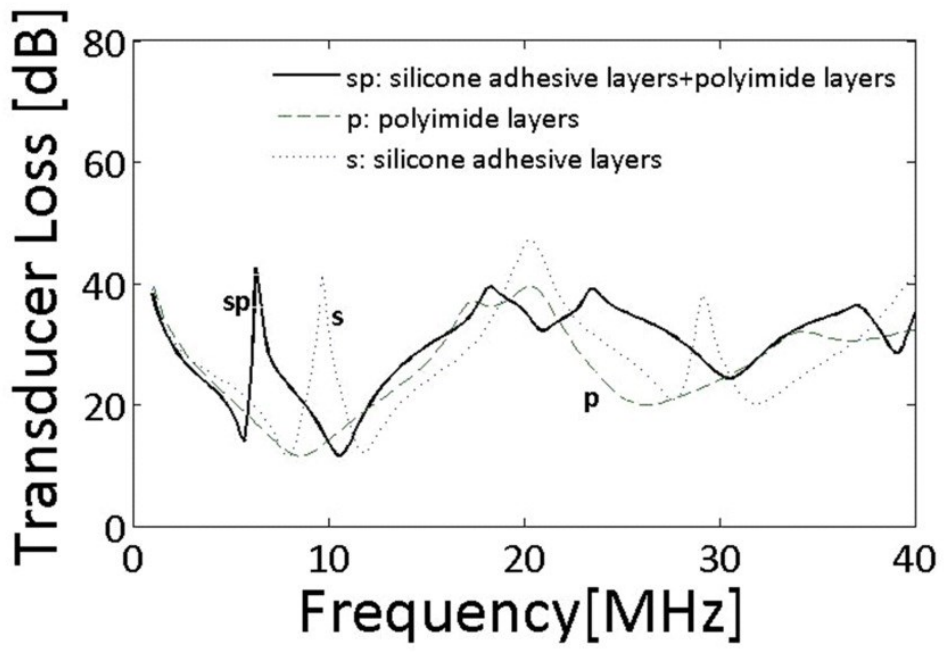

Figure 5.11: Calculated loss parameters of ultrasonic sensor composed of structures "sp", "s" and "p" ; (a) conversion loss (CL),(b) matching loss (ML), (c) transducer loss (TL). 
Finally, the PVDF sensor loss parameters were calculated by considering all the non-piezoelectric layers, as illustrated in Figure 5.12 where "esp" has a $26-\mu \mathrm{m}$ silver paint electrode layers, a 50- $\mu \mathrm{m}$ silicone adhesive layers, and $26-\mu \mathrm{m}$ polyimide film layers; "sp only" has 50- $\mu \mathrm{m}$ silicone adhesive layers, and $26-\mu \mathrm{m}$ polyimide film layers; "e" only has $26-\mu \mathrm{m}$ silver paint electrode layers. The conversion loss of the sensor with the full structure of "esp" was reduced at the dual operating frequencies of $5 \mathrm{MHz}$ and $8 \mathrm{MHz}$ by around $1 \mathrm{~dB}$ as seen in Figure 5.13(a), while the matching loss was increased by $1 \mathrm{~dB}$ at 8 $\mathrm{MHz}$ as seen inFigure 5.13(b). The silver paint electrode and polyimide film layers of the sensor structure "esp" caused the sensor to resonate at $6.3 \mathrm{MHz}$, and the silicone adhesive layers brought a large loss at the resonance frequency. Consequently, the sensor operates at the dual frequencies of $5 \mathrm{MHz}$ and $8 \mathrm{MHz}$. Also the bandwidth of the center frequencies became narrower comparing to the sensor of structure "sp", as seen in Figure 5.13(c). 


\begin{tabular}{|c|} 
Air \\
$\left(Z_{\mathrm{b}}\right)$ \\
\hline $\begin{array}{c}\text { Back Polyimide Film } \\
(13 \mu \mathrm{m})\end{array}$ \\
\hline $\begin{array}{c}\text { Back Silicone } \\
\text { Adhesive }(25 \mu \mathrm{m})\end{array}$ \\
\hline $\begin{array}{c}\text { Back Electrode } \\
(13 \mu \mathrm{m})\end{array}$ \\
\hline PVDF \\
$(110 \mu \mathrm{m})$ \\
\hline Front Electrode \\
$(13 \mu \mathrm{m})$
\end{tabular}

(esp)

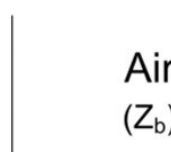

\begin{tabular}{|c}
\hline $\begin{array}{c}\text { Back Polyimide Film } \\
(13 \mu \mathrm{m})\end{array}$ \\
\hline
\end{tabular}

Back Silicone Adhesive $(25 \mu \mathrm{m})$

PVDF

$(110 \mu \mathrm{m})$

Front Silicone Adhesive $(25 \mu \mathrm{m})$



$(\mathrm{sp})$

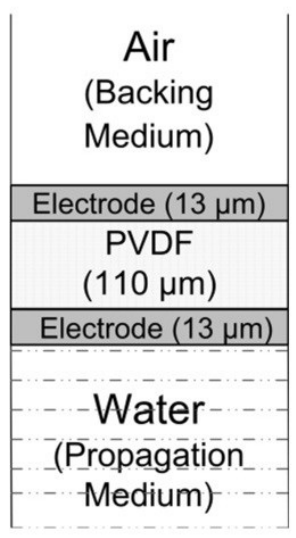

(e)

Figure 5.12: Structures of the three PVDF sensors composed of; (esp) electrode and protection layers, (sp) silicone adhesive and polyimide layers, (e) electrodes layers. 
(a)

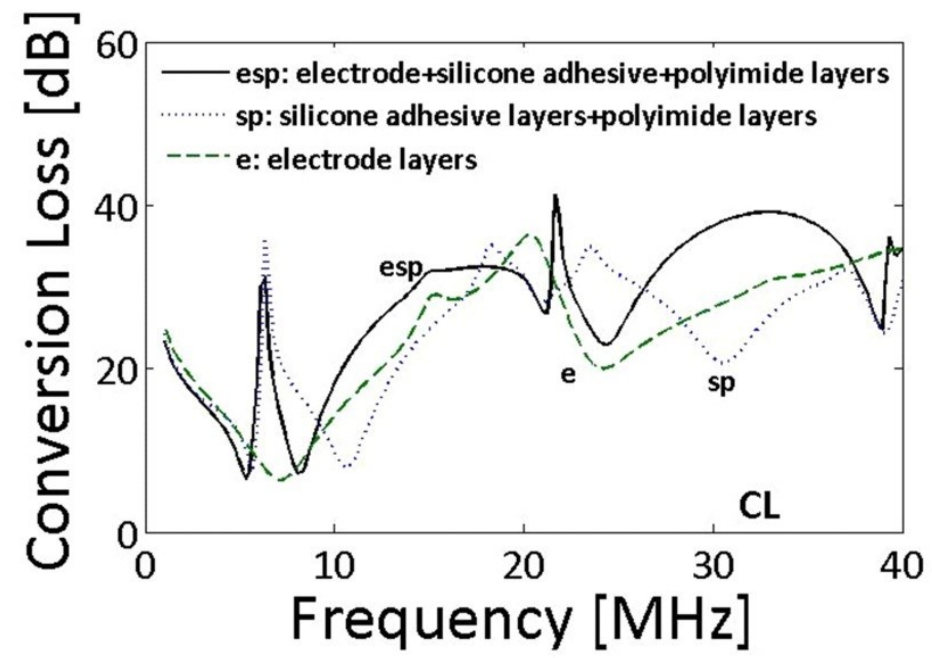

(b)

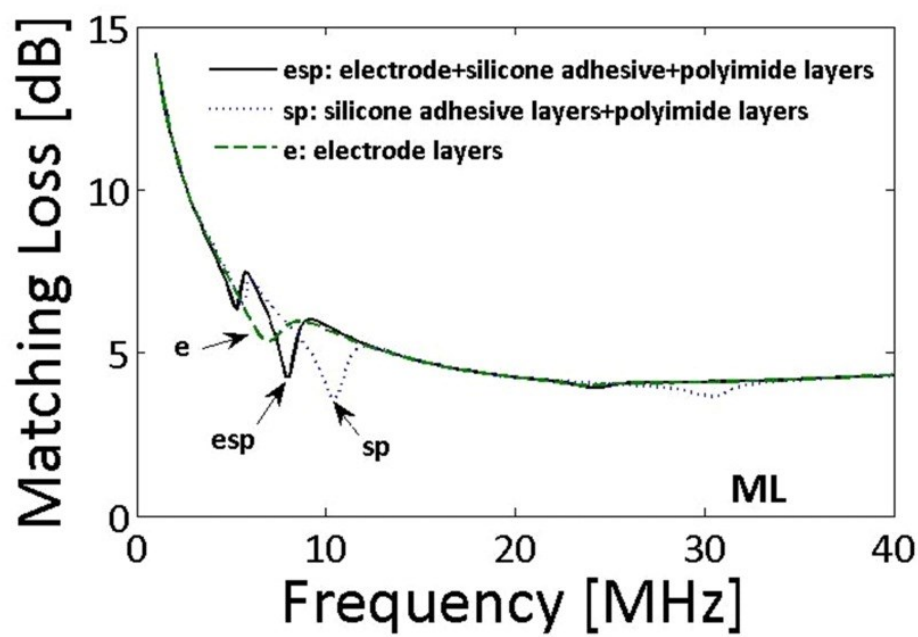

(c)

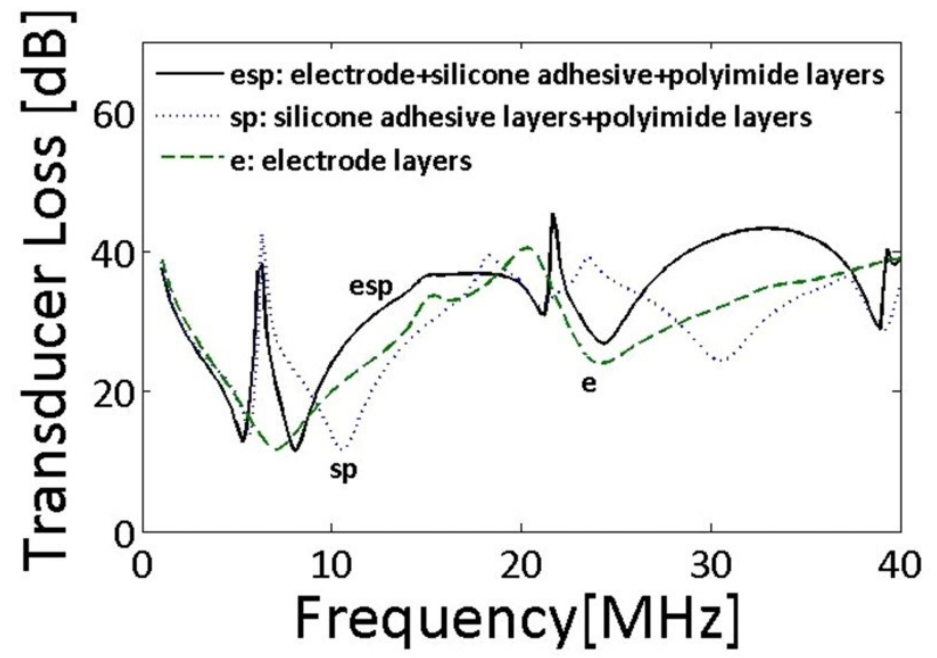

Figure 5.13: Calculated loss parameters of ultrasonic sensor composed of structures "esp", "sp" and "e" ; (a) conversion loss (CL), (b) matching loss (ML), (c) transducer loss (TL). 


\subsection{Experimental Investigation of Sensor Characteristics}

The developed sensor was evaluated theoretically by the numerical calculation in Section 5.1. In this section however, the characteristics of the developed sensor such as the frequency characteristics, the ultrasonic signal strength and the sensor size effect, were experimentally investigated.

\subsubsection{Frequency Characteristics}

The frequency characteristics of the developed PVDF sensor were experimentally investigated using an ultrasonic pulse echo technique. The sensor was constructed from $110-\mu \mathrm{m}$ thick PVDF sandwiched by $13-\mu \mathrm{m}$ thick silver paint electrodes and covered by the protection layer. The protection layer is a $13-\mu \mathrm{m}$ thick polyimide film glued by 25 $\mu \mathrm{m}$ thick silicone adhesive onto the both sides, as illustrated in the structure "esp" presented in Figure 5.12. The active area of the sensor, which is the overlapped area of the top and bottom electrodes, was $1.5 \mathrm{~cm} \times 1.5 \mathrm{~cm}\left(2.25 \mathrm{~cm}^{2}\right)$. The sensor was placed inside a water container which had a metal reflector at the bottom. To ensure that the sensor was air backed, the back side of the sensor was glued to a hollow Plexiglas tube, as shown in Figure 5.14. The area of the tube's open end was considered to be larger than the active area of the sensor to ensure the sensor was fully air backed. During the experiment, the sensor was held by a mechanical stage (Model: BiSlide, Velmex Inc., Bloomfield, NY, USA) allowing a vertical movement of the sensor inside the water container. The sensor was driven by an ultrasonic pulser/receiver (DPR300, JSR), and the signal reflected from the reflector was acquired. The ultrasonic signals were recorded at 
different distances between the sensor and the reflector $(4,24,32,48 \mathrm{~mm})$ as shown in Figure 5.15. The reflected signals were acquired by a PCI digitizer (ATS 460, AlazaraTech,) with a $125 \mathrm{MHz}$ sampling rate and a $10 \mathrm{~Hz}$ ultrasonic pulse repetition rate.
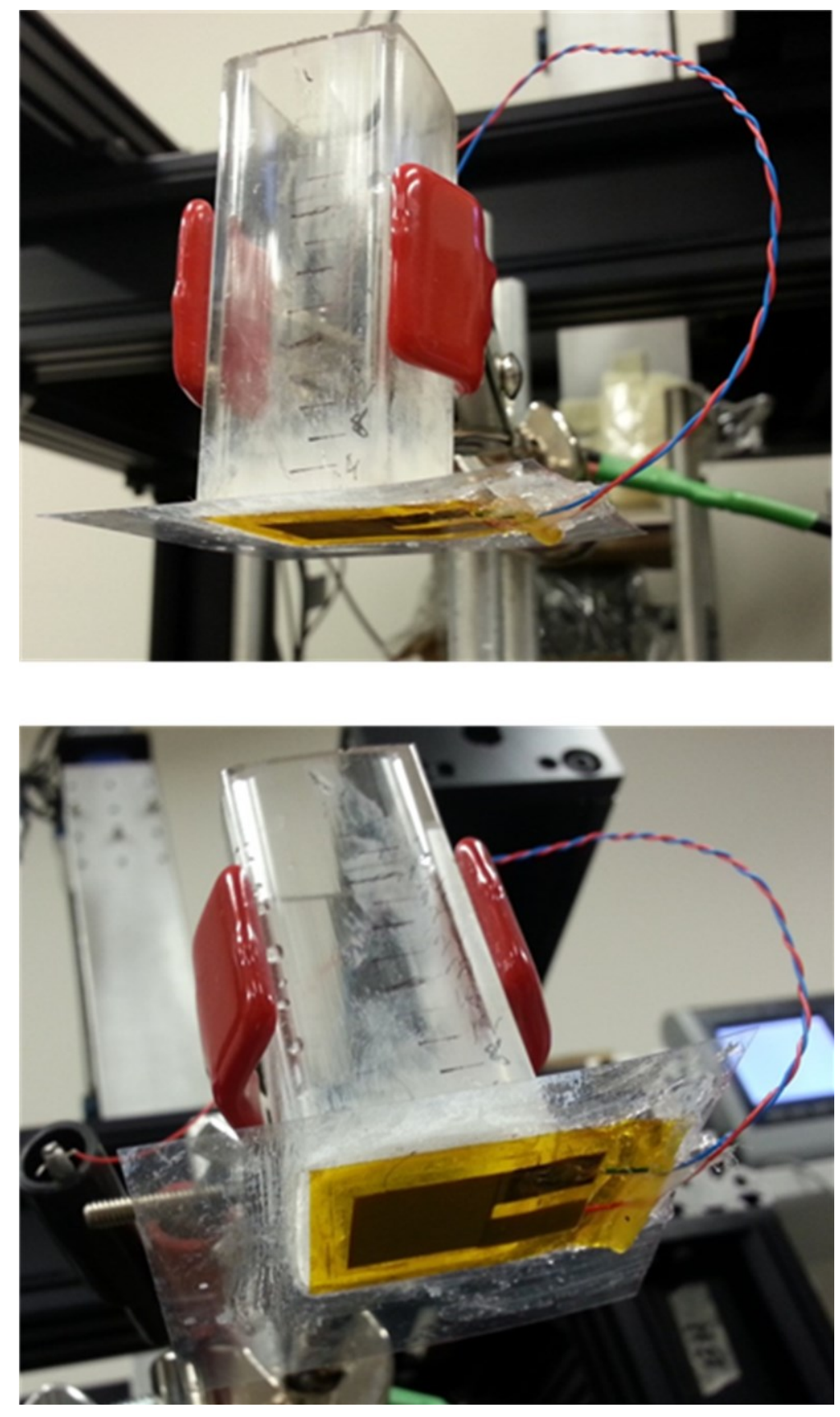

Figure 5.14: Photo of sensor glued to a Plexiglas tube. 


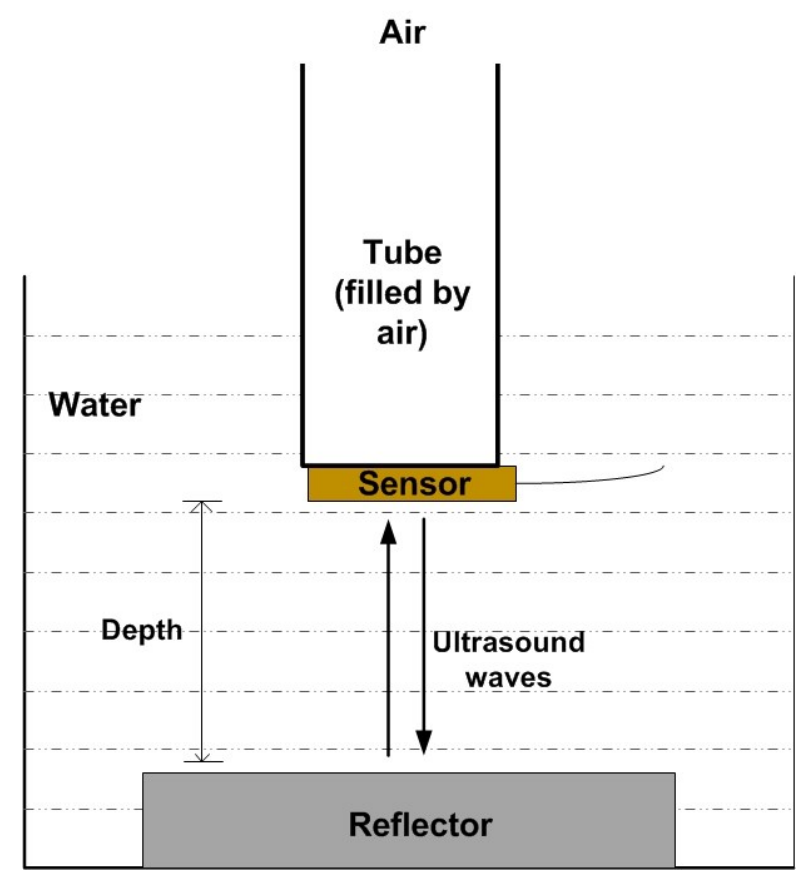

Figure 5.15: Sketch of the experiment procedure.

Figure 5.16 shows the frequency spectra of the reflected ultrasonic signal obtained by the developed sensor using a pulse-echo technique, at several depths from the reflector. The frequency response presented in Figure 5.16(a)-(d) shows that the sensor operates at the dual frequencies of $3.8 \mathrm{MHz}$ and $7.6 \mathrm{MHz}$ as predicted from the simulation results presented in Figure 5.13. However, the differences of the dual frequency values between the numerical calculation (5 and $8 \mathrm{MHz}$ ) and the experimental result (3.8 $\mathrm{MHz}$ and 7.6 $\mathrm{MHz}$ ) are possibly due to the differences between the mechanical properties of the sensor materials used in the experiment, and the parameters of those used in the numerical calculation. The venders were not able to provide the exact properties of the purchased materials so they were obtained from several sources as listed in Table 5.1. The amplitude of the dual frequencies decreased with depth because the transmitted ultrasound attenuates more when travelling longer distance in water. 


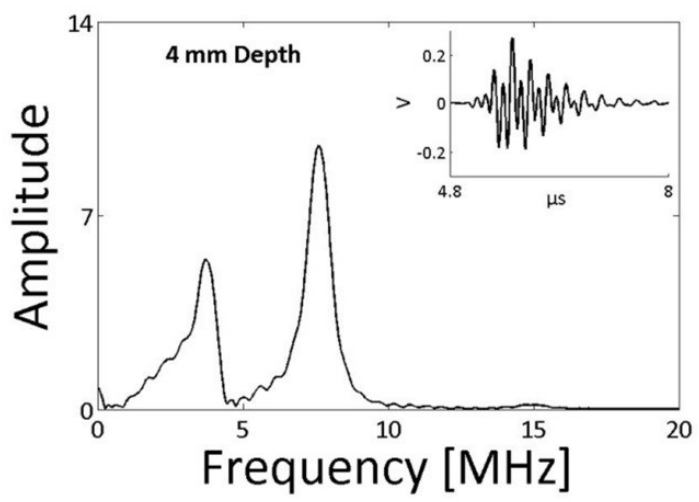

(a)

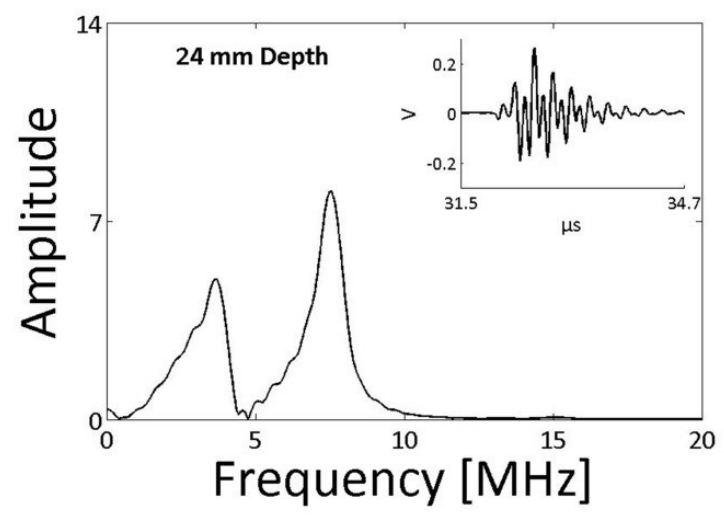

(c)

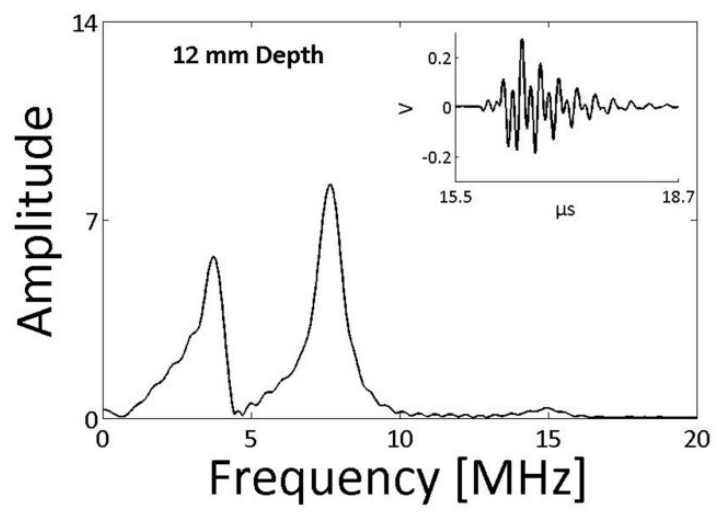

(b)

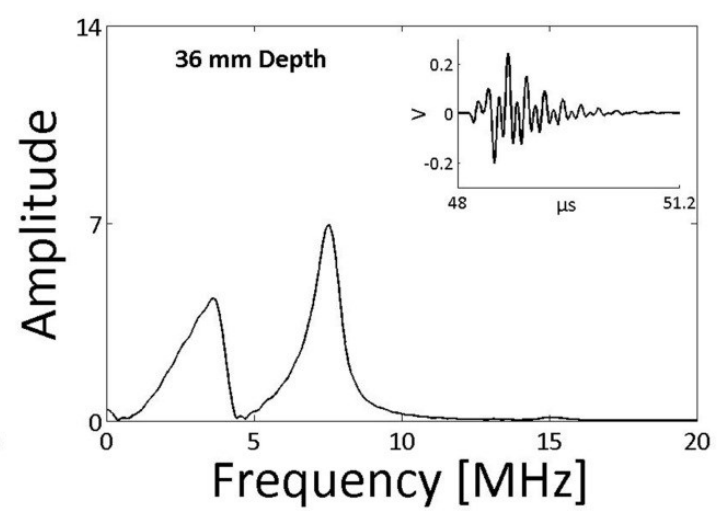

(d)

Figure 5.16: Frequency spectrum of the reflected ultrasonic pulse signal acquired by the developed sensor at several depths from the reflector; (a) $4 \mathrm{~mm}$, (b) $12 \mathrm{~mm}$, (c) $24 \mathrm{~mm}$ and (d) $36 \mathrm{~mm}$. 


\subsubsection{Ultrasonic Signal Strength}

This section discusses the ultrasonic signal strength of the developed sensor. An experiment was conducted to study the ultrasonic signal strength with respect to the ultrasonic active size (electrode size). Five sensors with various active area sizes $(0.25$, $1.00,2.25,4.00$ and $6.25 \mathrm{~cm}^{2}$ ) as shown in Figure 5.17 were constructed to compare their ultrasonic signal strength. All the sensors have the same structure as presented in Figure 5.12(esp). The sensors were attached onto a $12.5-\mathrm{mm}$ thick Plexiglas plate sample, and the ultrasonic signal reflected from the bottom surface of the sample was acquired as illustrated in Figure 5.18. A double-sided polyimide tape with silicone adhesive on both sides was used as an ultrasonic couplant in this experiment instead of the conventional ultrasonic gel couplant to avoid the uneven thickness distribution of the couplant between the sensor and the sample. It was found that non-uniformity of the couplant thickness significantly affects the ultrasonic signal strength. The silicone adhesive in the doublesided polyimide tape allows an even distribution of the couplant thickness over the area of the attachment between the sensor and the sample. Figure 5.19 shows the measured signal strengths at the center frequency of 7.6 MHz. The acquired signals were averaged 100 times to improve the signal-to-noise ratio. The results in Figure 5.19 indicate that the signal strength increases with the sensor size under the experimental conditions and sensor configuration employed in this study. Thus, the results demonstrate the importance of considering a suitable sensor size for the measured area of interest. A larger sensor

area provides a greater signal strength but inferior spatial resolution in the lateral direction. 


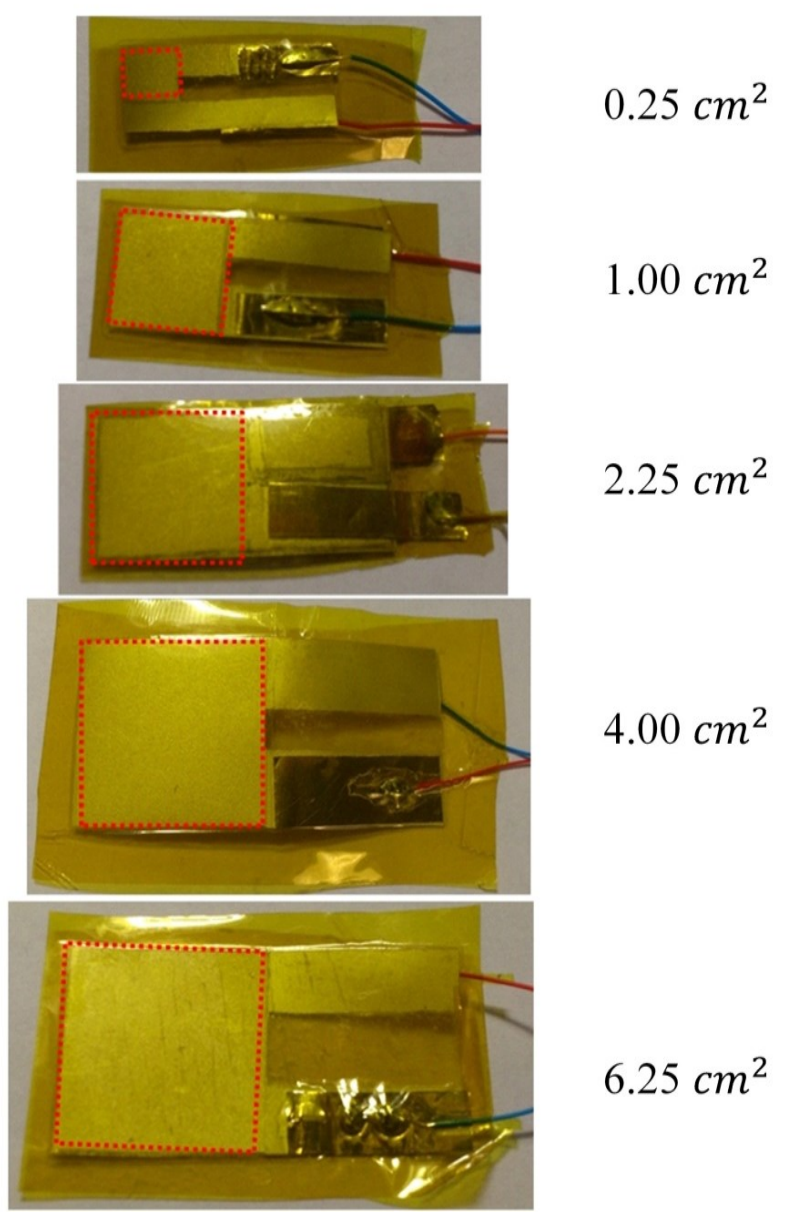

Figure 5.17: Photo of ultrasonic sensor having different active areas size.



Figure 5.18: Schematic of experiment configuration for evaluation of ultrasonic signal strength with respect to the sensor active area size. 


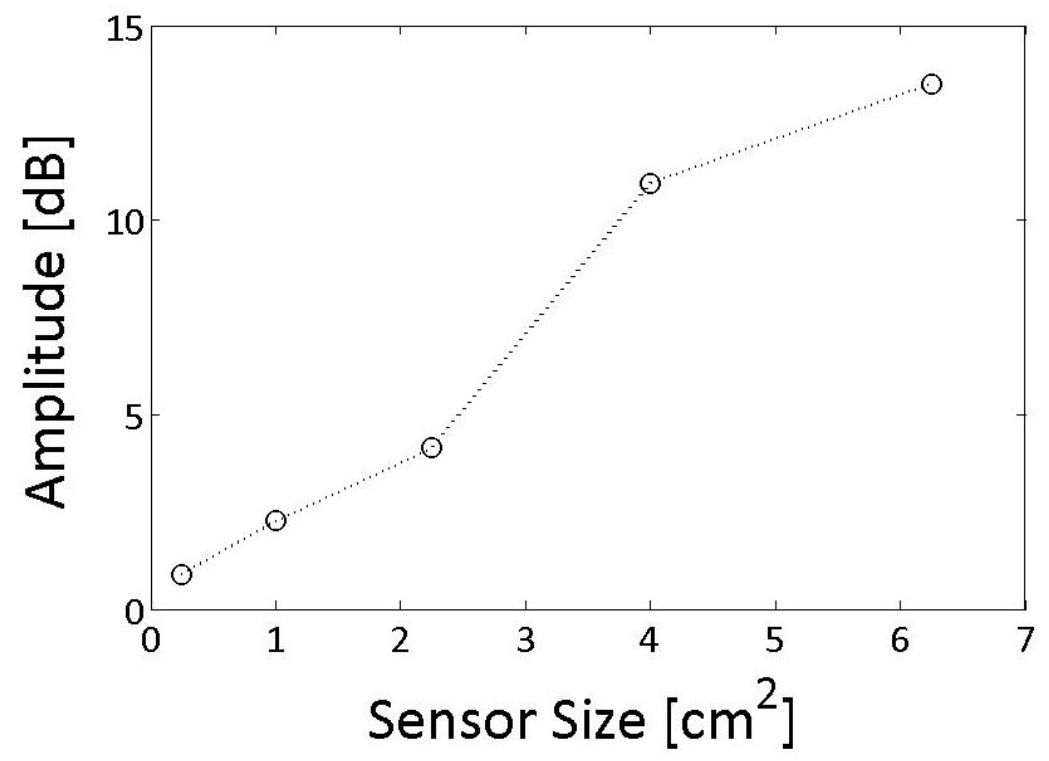

Figure 5.19: Ultrasonic signal strength comparison between various sensor sizes.

The electrical impedance matching between the electric power source (a transmitter of ultrasonic pulser/receiver) and the ultrasonic sensor allows a maximum power transfer from the source to the sensor. Thus, it is ideal if the source output impedance equals the sensor electrical impedance. Since the electrical impedance of the sensor depends on the sensor size, the relationship between the sensor electrical impedance and sensor size is investigated by numerical simulation.

The matching loss (ML) was calculated for several sensor sizes $\left(0.25\right.$ to $\left.30 \mathrm{~cm}^{2}\right)$ with different source impedance values $(50,100,300,500$ and $1000 \Omega)$. Figure 5.20 illustrates the matching loss at the resonance frequency of $8 \mathrm{MHz}$ for several sensor sizes with respect to the electric source impedances. The minimum loss value indicates a better match between the sensor's electrical impedance and the source impedance. These results show that the sensor impedance decreases as the sensor size increases. The sensor best 
impedance match for the sensor is at a size of $8 \mathrm{~cm}^{2}$ with a $50 \Omega$ source impedance, while the matching point shifts to a $0.5 \mathrm{~cm}^{2}$ sensor size with a $1000 \Omega$ source impedance. Hence, it's important to consider the sensor impedance as a factor when choosing the sensor size and considering the source impedance.

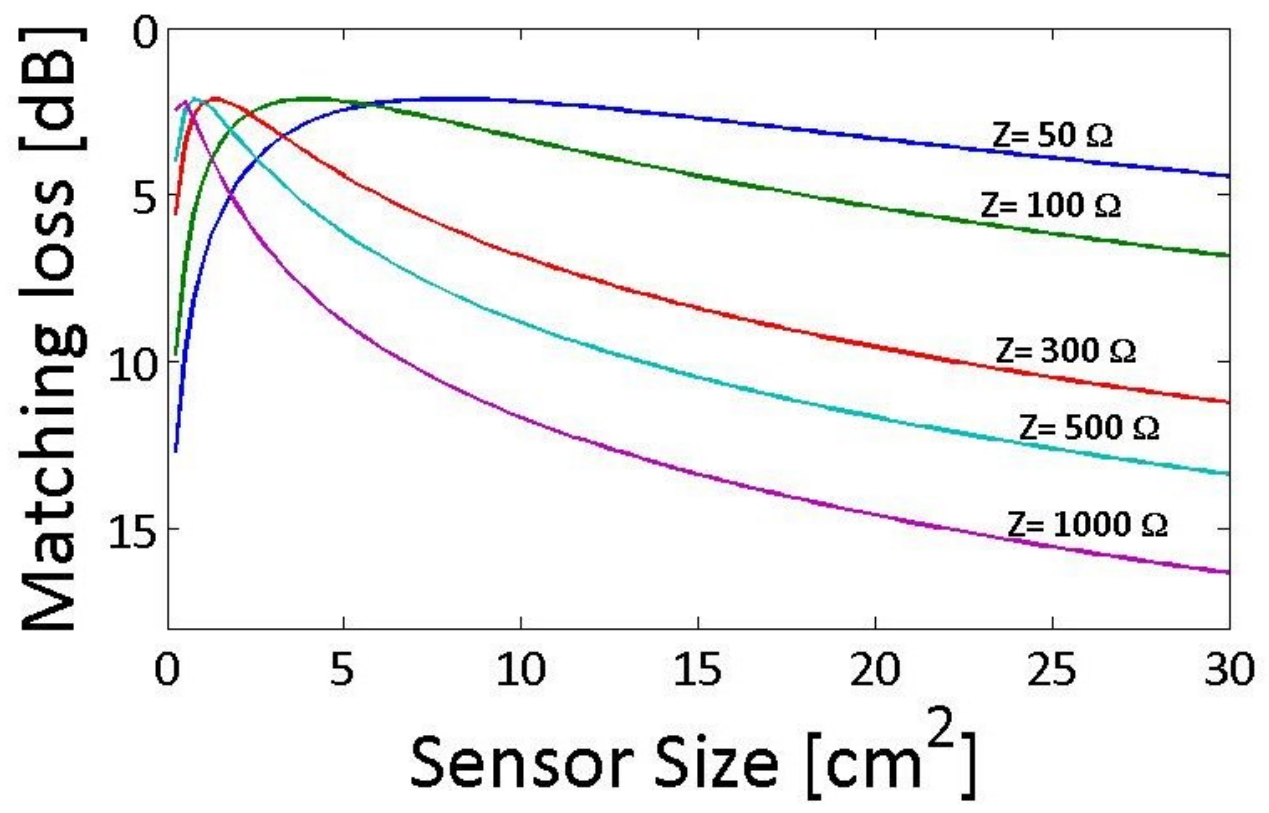

Figure 5.20: Matching loss for several sensor sizes at the resonant frequency $8 \mathrm{MHz}, \mathrm{Z}$ is the source impedance. 


\subsubsection{Backing Material to Improve Frequency Characteristics}

The evaluation of the frequency characteristics of the first design of the developed sensor (air backed PVDF sensor with electrode and protection layers) showed that the sensor operates in dual frequencies. As the backing material plays an important role in the frequency response of the sensor, a second design of the ultrasonic sensor with a backing material, was constructed in order to improve its frequency response.

Silicone rubber was selected as the backing material due to its large attenuation coefficient and its matching acoustic impedance to the PVDF. The 1-mm thick silicone rubber was glued directly to the back electrode using silicone, as presented in Figure 5.21. The silicone rubber also serves as a protection/insulation layer. The sensor's flexibility is still preserved with the silicone rubber backing.

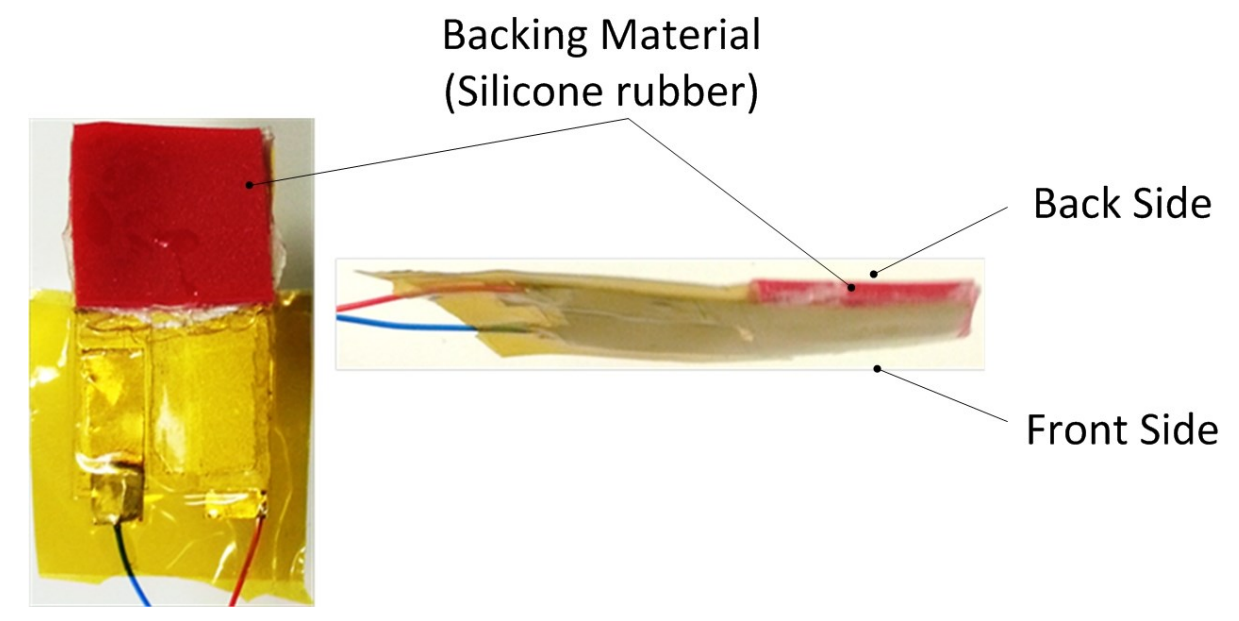

Figure 5.21: Photo of the ultrasonic film sensor developed with the backing material. 
Figure 5.22 shows the calculated losses parameters (conversion loss, matching loss and transducer loss) of the silicone backed sensor with an infinite thickness of the silicone backing layer. The sensor structure is shown in Figure 5.22(a). The propagation medium was assumed to be water. Figure 5.22(b) shows that the silicone backing changed the frequency response of the sensor to a single operating frequency of $7.2 \mathrm{MHz}$ with a broader frequency bandwidth. The comparison of the transducer loss between the air backing sensor (structure esp) and the sensor with silicone backing material is illustrated in Figure 5.23.

In addition, the frequency response of the silicone backed sensor was experimentally examined using the sensor in Figure 5.21. The sensor was placed inside a water container with the experimental configuration shown in Figure 5.14 and Figure 5.15. The reflected ultrasonic signal from the container bottom was acquired using an ultrasonic pulse echo technique. Figure 5.24 shows the frequency spectrum of the acquired ultrasonic signal. As expected from the numerical calculation results in Figure 5.22(b), the operating frequency of the silicone backed sensor became broader (6$\mathrm{dB}$ bandwidth is $7 \mathrm{MHz}$ ) at the operating frequency of $7.6 \mathrm{MHz}$. 


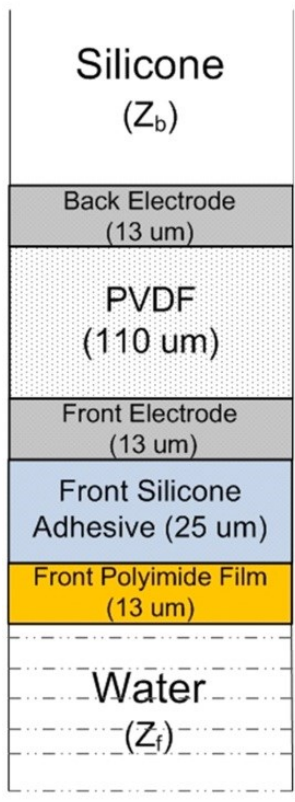

(a)

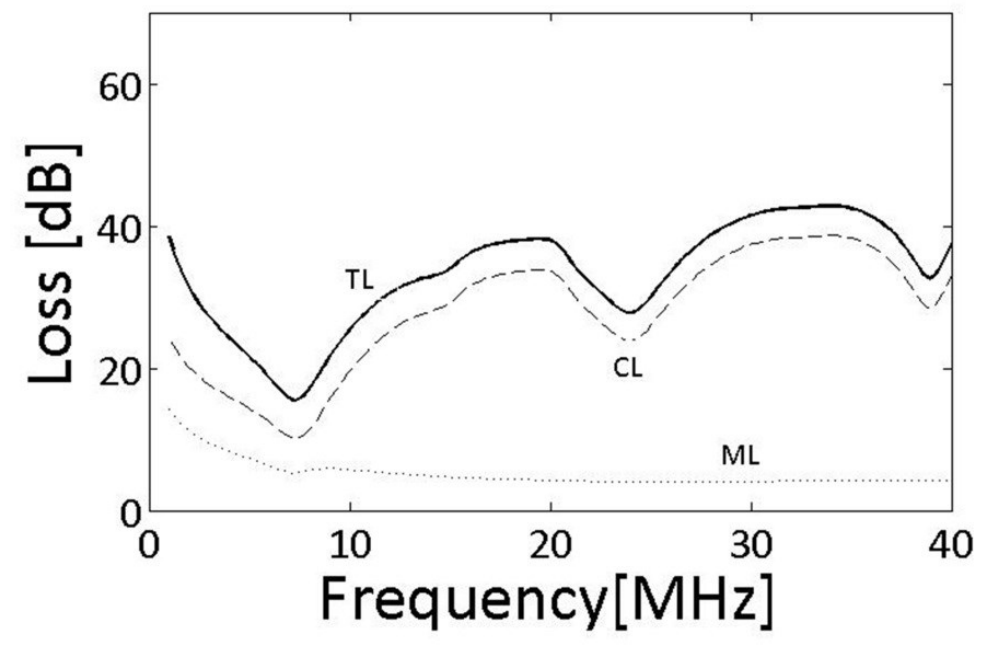

(b)

Figure 5.22: Calculated loss parameters of ultrasonic sensor composed of $110-\mu \mathrm{m}$ thick PVDF film backed with silicone; (a) sensor structure, (b) losses (TL+CL+ML).

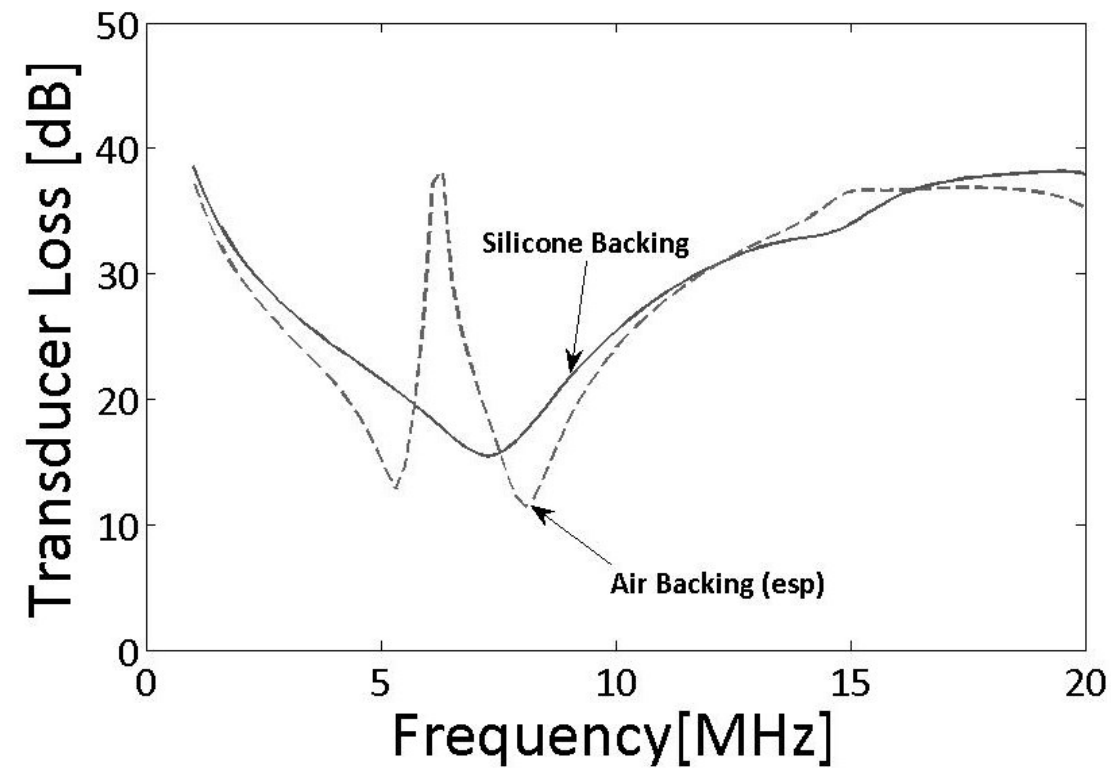

Figure 5.23: Calculated Transducer loss of are backing sensor and silicone backing sensor. 


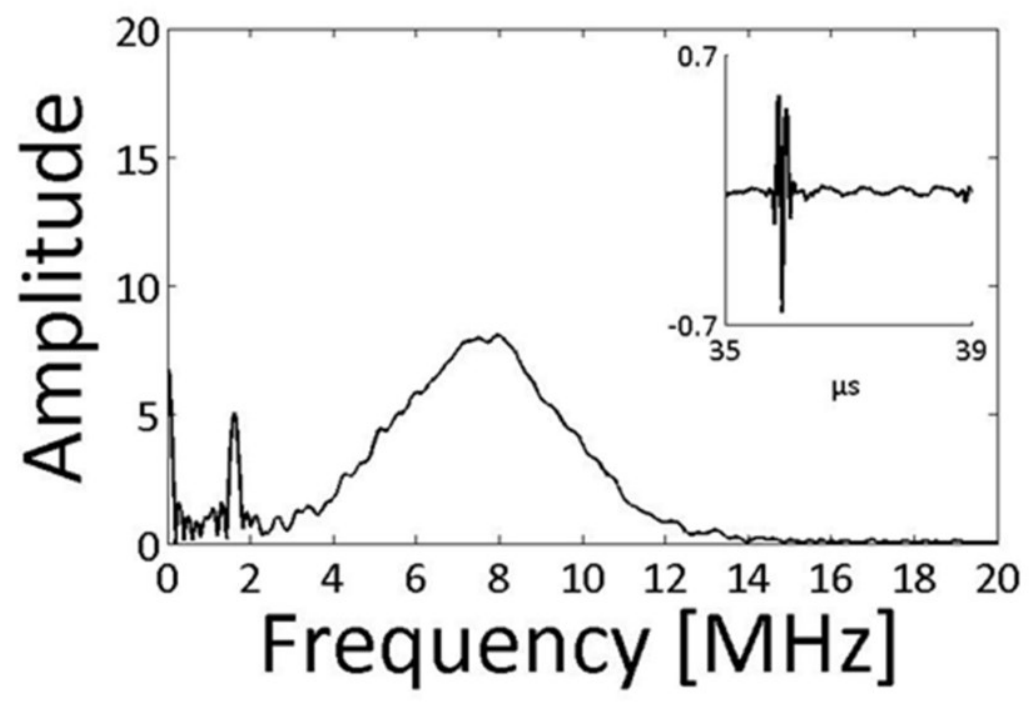

Figure 5.24: Frequency spectrum of the reflected signal pulse acquired with the sensor having a silicone backing. 


\subsection{Couplant Selection and Evaluation}

In the procedure of monitoring muscle activity using the developed ultrasonic sensor, a couplant is needed to mediate the ultrasound transmission between the sensor and the biological tissue. In addition, the couplant fills the irregularities of the interface and prevents air gaps between the two surfaces [61].

The muscle monitoring application requires a couplant that maintains the sensor stability on the surface of the body and preserves its performance during a long-term measurement. Therefore, several materials were chosen in order to investigate their performance as couplants. The tested materials were: a medical grade ultrasonic gel, honey, paper glue, and double-sided polyimide adhesive tape. Each couplant was tested with the developed ultrasonic sensor using a $12.5-\mathrm{mm}$ thick Plexiglas plate sample with the same conditions for the ultrasonic pulsar/receiver, such as the electrical pulse energy, the dumping, the amplification, and the band pass filter. The couplants' effectiveness was evaluated by measuring the ultrasonic signal strength that was reflected from the bottom of the Plexiglas sample. The results are shown in Figure 5.25. It was observed that the paper glue and the polyimide tape had slightly stronger amplitudes than the ultrasonic gel. However, honey was the weakest among them and had a $13 \%$ smaller amplitude value than the ultrasonic gel.

A further investigation was made to evaluate the couplants' effectiveness for a long-term continuous measurement. The ultrasonic signal reflected from the bottom of the Plexiglas sample was acquired every 15 seconds, for 24 hours for each couplant. The honey was not included in the test due to its weak signal performance among the chosen materials, as discussed above. Figure 5.26 presents the results. The three couplants tested 
were capable of lasting more than 24 hours as ultrasonic couplants. However, the amplitude value with the ultrasonic gel couplant decreased for the first six hours, and then started increasing to a value $7.5 \%$ higher than the initial one. Likewise, the paper glue showed a rapid decrease in the first hour, and then started to increase constantly. The possible reason for the decrease in amplitude values during the first hours may be the solidification of the glue and the drying of the ultrasonic gel. In contrast, the amplitude of the polyimide tape increased constantly for 17 hours, and then became almost constant.

The polyimide tape has a better long-term stability and durability compared to the ultrasonic gel and the paper glue. In addition, the polyimide tape may make the sensor adhere to the skin surface instantly. It also prevents the sensor's movement and provides a constant thickness of couplant during the measurement. Therefore, the double-sided polyimide tape may have the advantage among the chosen materials in terms of stability and durability with the conditions employed in this experiment.

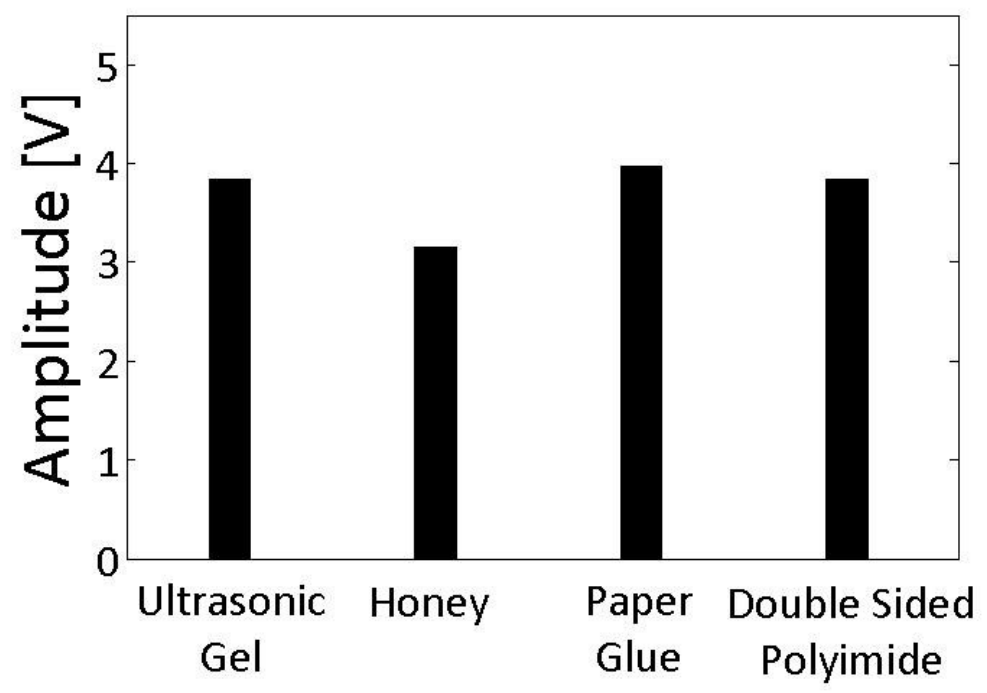

Figure 5.25: Measured echo signal amplitude with the four different couplants: ultrasonic gel, honey, paper glue, and double-sided Polyimide tape. 


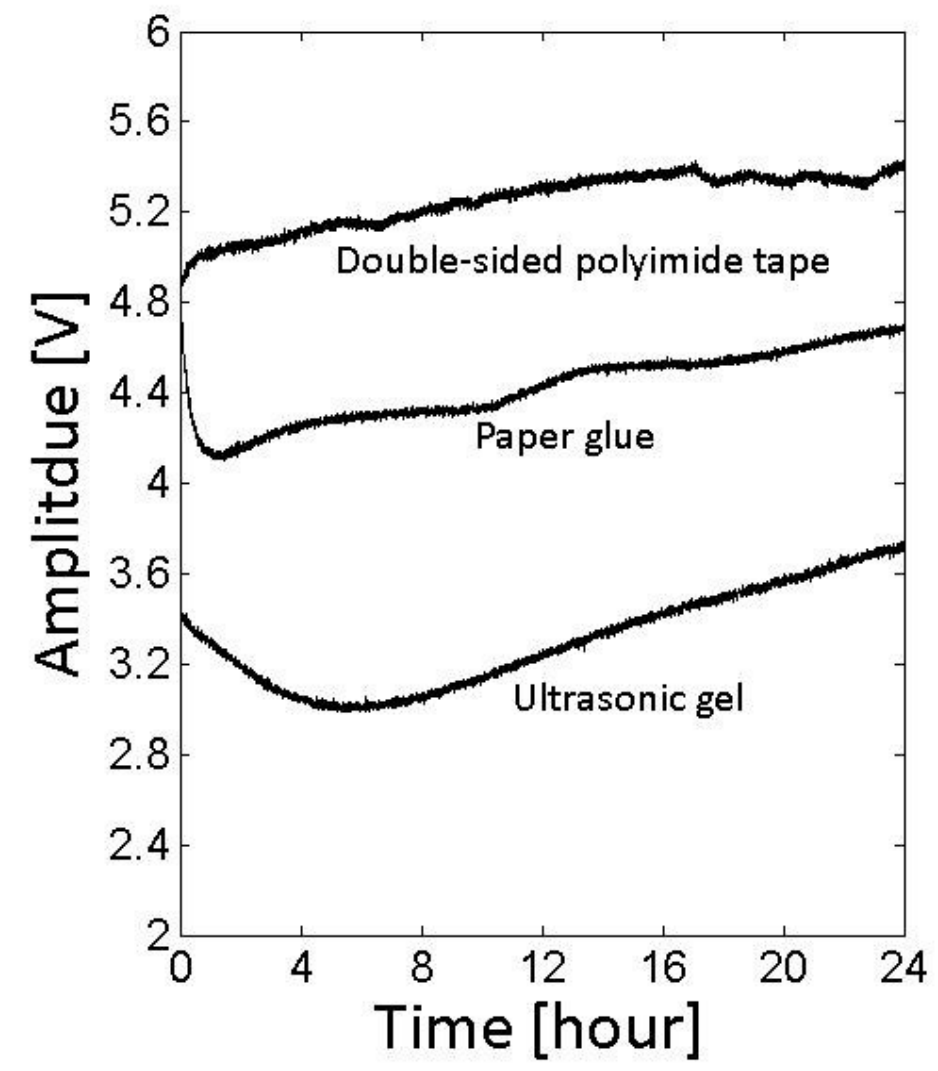

Figure 5.26: The reflected ultrasonic signal amplitude variation over 24 hours for the ultrasonic gel, paper glue, and double-sided polyimide tape. 


\section{Chapter 6: Muscle Monitoring}

This chapter demonstrates the use of the developed wearable sensor to monitor muscle contractions. The sensor has been applied to measure the change in the thickness of the human tissue during static and dynamic contractions using the ultrasonic pulseecho and through-transmission techniques. An experiment to investigate the ultrasonic penetration depth is also presented using a human subject.

\subsection{Contraction of Forearm}

This section demonstrates the measurement of thickness variations of the forearm tissue during an isotonic contraction. The objective of this experiment is to monitor the forearm muscle using the developed sensor with the ultrasonic techniques of pulse-echo and through-transmission.

\subsubsection{Pulse-Echo Technique}

This experiment has been conducted to measure the thickness of the forearm tissue during an isotonic contraction by the developed sensor using the pulse-echo technique. In the pulse-echo technique, a single ultrasonic sensor serves as a transmitter as well as a receiver. The bone reflects the transmitted ultrasonic signal back to the sensor since the bone has a higher acoustic impedance $\left(7.75 \times 10^{6} \mathrm{~kg} / \mathrm{m}^{2} \mathrm{~s}\right)$ [62] compared to soft tissue $\left(1.63 \times 10^{6} \mathrm{~kg} / \mathrm{m}^{2} \mathrm{~s}\right)[54]$, resulting in a reflection of $42.5 \%$ of the ultrasound energy 
at the interface of the soft tissue and bone. The distance between the sensor and the bone changes during contraction due to the muscle thickness change. The developed sensor was attached to the superior side of the forearm, $3-\mathrm{cm}$ away from the wrist joint, by adhesive tape, as shown in Figure 6.1. The adhesive tape was applied carefully to avoid the exertion of force upon the forearm surface by the sensor, which it may restrict the muscle motion. The ultrasonic signal reflected from the interface between the muscle tissue and the radius bone was acquired for 10 seconds while the muscles beneath the sensor were contracting voluntarily by bending the wrist joint backward every 2 seconds. The bone echo was clearly observed in the signals received, as shown in Figure 6.2. The tissue's thickness $\left(d_{T}\right)$ between the sensor and the bone was calculated using the time delay $\left(t_{u s}\right)$ of the reflected signals by $d_{T}=v \times \frac{t_{u s}}{2}$, where $v$ is the mean ultrasound velocity in the human tissue $(1540 \mathrm{~m} / \mathrm{s}$ [54]). The result is plotted over the total measured time (10s), as seen in Figure 6.3. Also, the M-mode image illustrating the muscle thickness change is shown in Figure 6.4. The thickness of the tissue at the relaxed state (where the wrist was not bending) was around $7.7 \mathrm{~mm}$, while the thickness was around $8.1 \mathrm{~mm}$ at the contracted state. The tissue thickness varied during the contraction around $400 \mu \mathrm{m}(5 \%)$, in accordance with the isotonic muscle contraction preformed.

It is noted that tracking the peak location of the ultrasonic signals reflected from the bone during the dynamic contraction counters a challenge. The movement of the subject affects the alignment of the sensor with the bone, which sometimes results in missed echoes from the bone. 
(a)

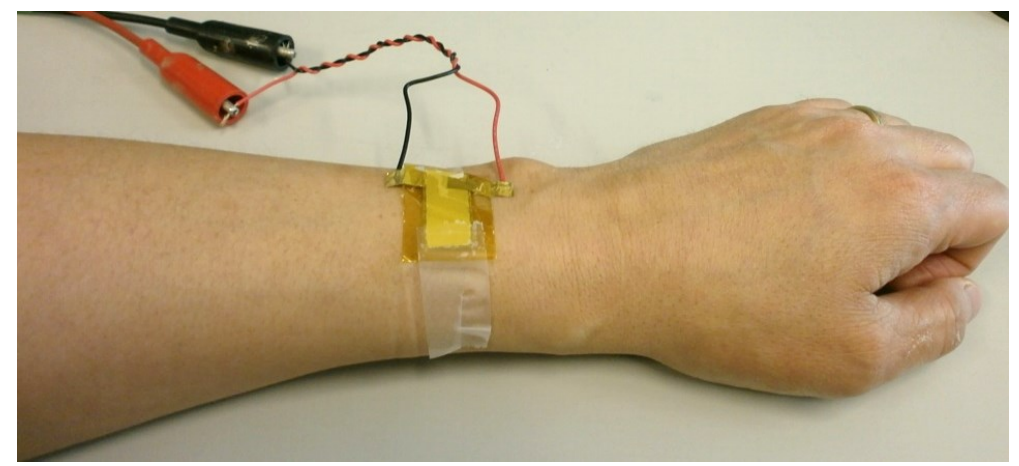

(b)

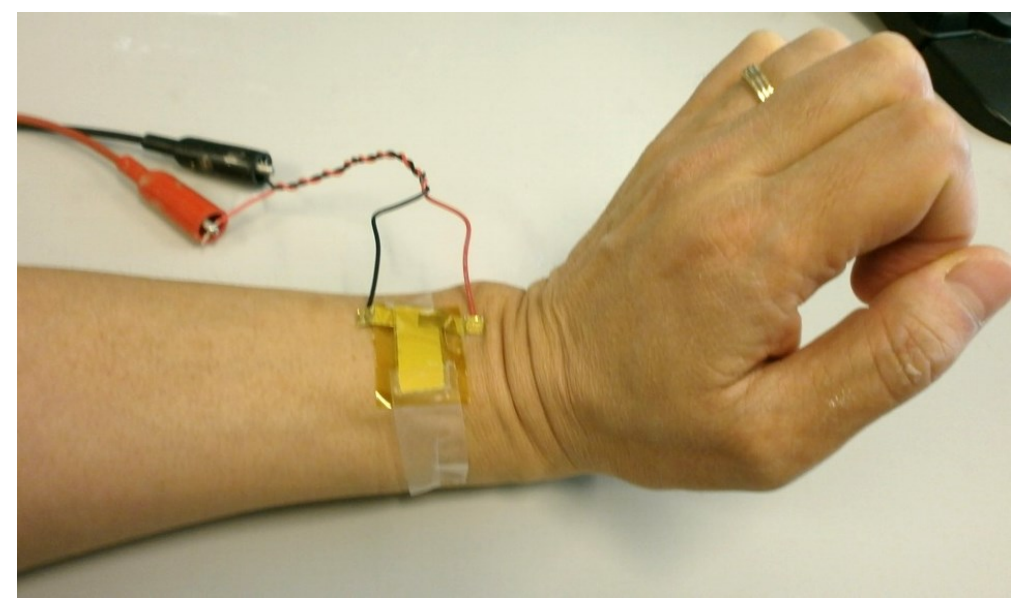

Figure 6.1: Wearable ultrasonic sensor attached onto forearm to monitor isotonic muscle contraction using pulse-echo technique, (a) at relaxed and (b) at contracted state.

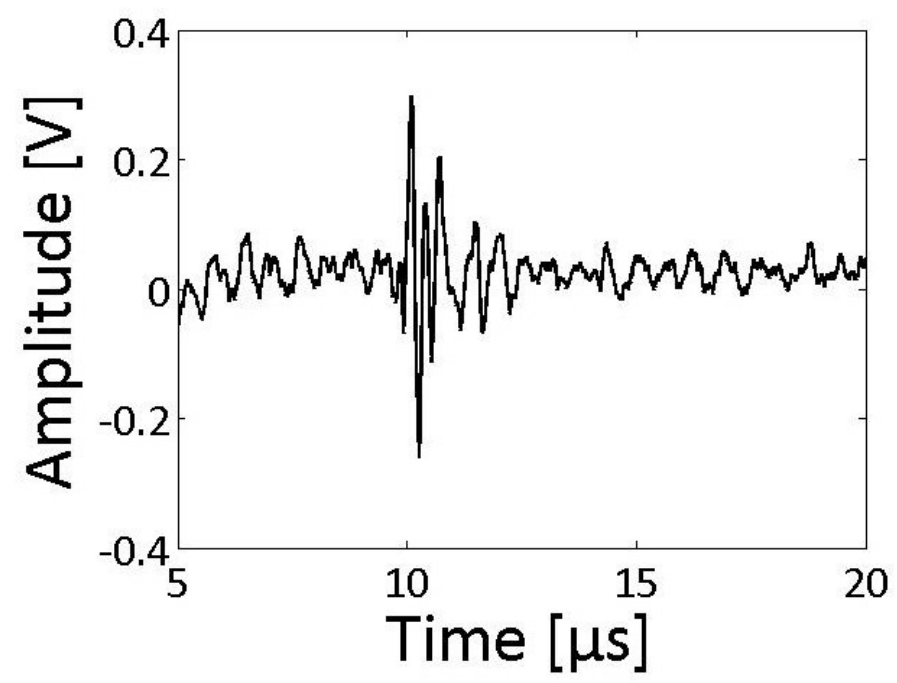

Figure 6.2: Ultrasonic single reflected from the tissue-bone interface at the forearm. 


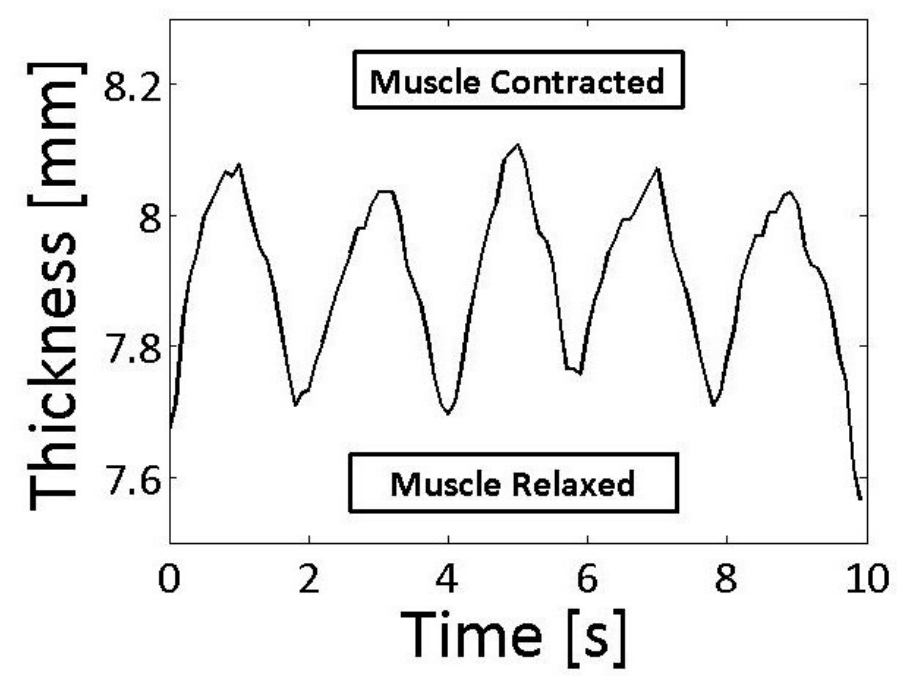

Figure 6.3: Thickness variation during forearm muscle contraction monitored by the developed sensor.



Figure 6.4: M-mode image of ultrasound measurement of forearm tissues by pulse-echo technique. 
In order to verify the ultrasonic signals reflected from the bone that were obtained by the developed sensor, during the isotonic contraction a B-mode image of the crosssection of the forearm was acquired using a clinical ultrasound imaging system (Model: PICUS, ESAOTE, Maastricht, Holland). The subject's forearm was submerged in a water bath to perform the ultrasound measurement with layer of water between the ultrasonic probe and the subject. The probe was positioned above the subject without contacting the subject, as shown in Figure 6.5. The B-mode images were acquired during the relaxed and contracted states. The subject contracts the forearm muscle by lifting the palm backwards. The B-mode images are shown in Figure 6.6. These images illustrate the increased muscle thickness of around $752 \mu \mathrm{m}$, between the skin and the bone during the contraction. 
(a)

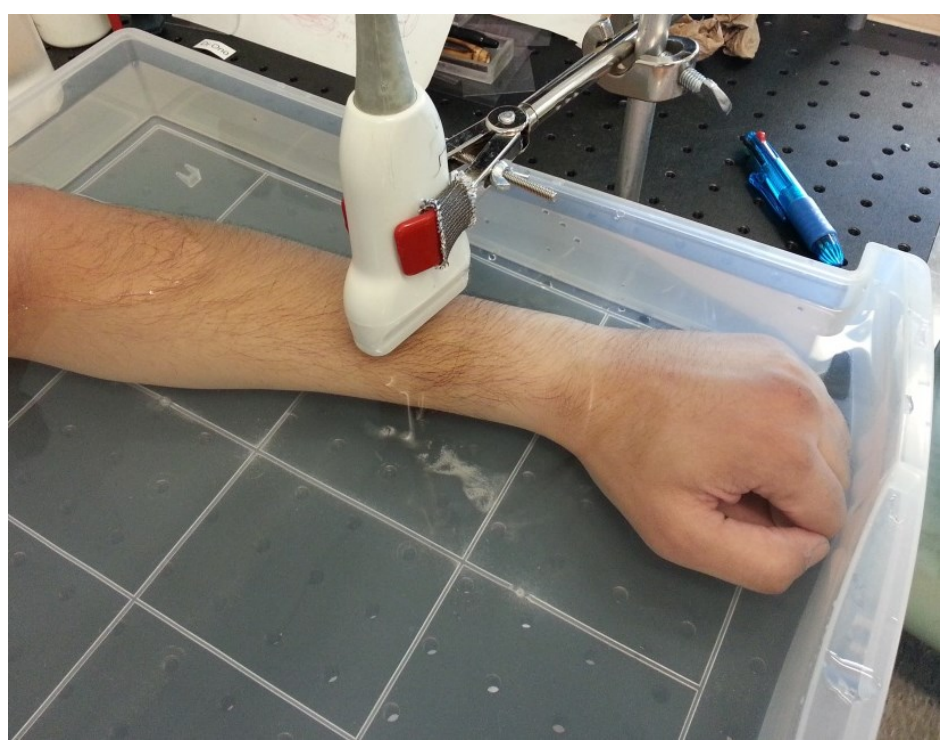

(b)

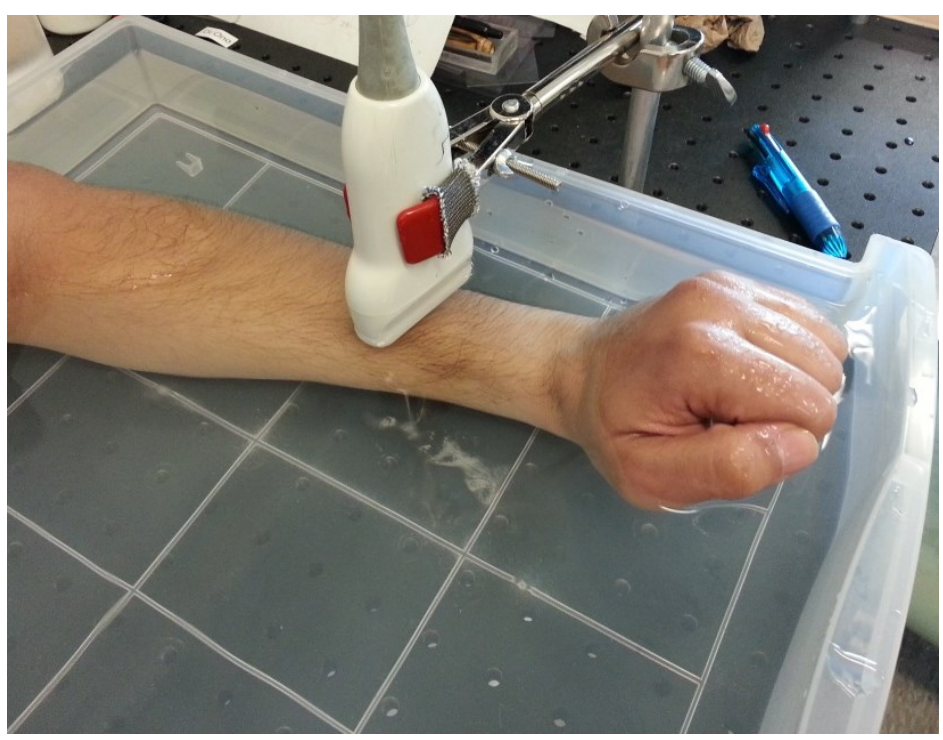

Figure 6.5: The setup of the experiment with the clinical ultrasound imaging system, (a) at relaxed state, (b) at contracted state. 
(a)

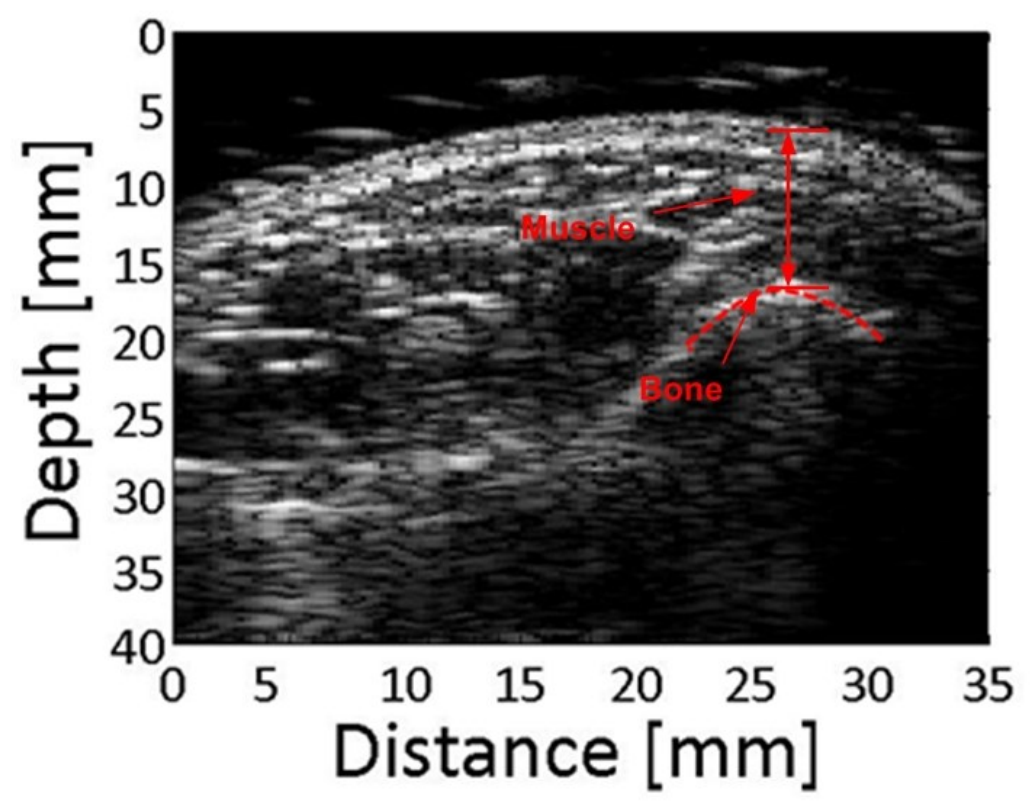

(b)



Figure 6.6: B-mode images of forearm with the clinical ultrasound imaging system. The target muscle of interest and bone is shown in red, (a) at relaxed state, (b) at contracted state. 


\subsubsection{Through-Transmission Technique}

In this experiment, the isotonic contraction of the forearm muscle was monitored using the through-transmission technique. In this technique, two sensors were used to measure the tissue thickness; one as a transmitter and the other as a receiver. The tissue thickness was calculated using the time delay of the received ultrasonic signal by $d_{T}=v \times t_{u s}$. The choice of which technique to use (through-transmission or pulseecho) depends on the accessibility of the muscle to be measured. The throughtransmission allows a measurement of the muscle contraction without a bone to reflect the ultrasonic signal. Also, with through-transmission, the distance travelled by the ultrasound is equivalent to the thickness of the subject's tissue, while with pulse-echo, the distance travelled is equivalent to twice the thickness of the subject's tissue. This results in a smaller attenuation of the ultrasonic signal. Thus, it allows the monitoring of thicker tissues.

The transmitter sensor was attached to the posterior side of the forearm $6-\mathrm{cm}$ away from the wrist joint and between the ulna and radius bones, whereas the receiver sensor was placed on the opposite side (anterior side), as shown in Figure 6.7. In this experiment, the ultrasonic signals generated from the transmitter sensor propagate through the soft tissue toward the receiver sensor while the muscle between the two sensor were contracting voluntarily by bending the wrist joint backward about every 2 seconds. The ultrasonic signals received, which passed through the tissues are presented in Figure 6.8. The ultrasonic signals were clearly observed at a time of $32.5 \mu \mathrm{s}$. The Figure 6.9 shows the calculated change of tissue thickness during the contraction. The thickness change was about $1 \mathrm{~mm}$. Also, the M-mode image illustrates the muscle 
thickness change over the measurement time, as shown in Figure 6.10. The thickness of the tissue at the relaxed state (where the wrist was not bending) was around $44.5 \mathrm{~mm}$, and it increased to a maximum of $45.5 \mathrm{~mm}$ during contraction.

It is noted that the alignment of the transmitter and the receiver sensor on the opposite sides of the forearm counters a challenge. The two sensors must be carefully aligned face-to-face and in parallel in order to receive the transmitted sound waves efficiently.
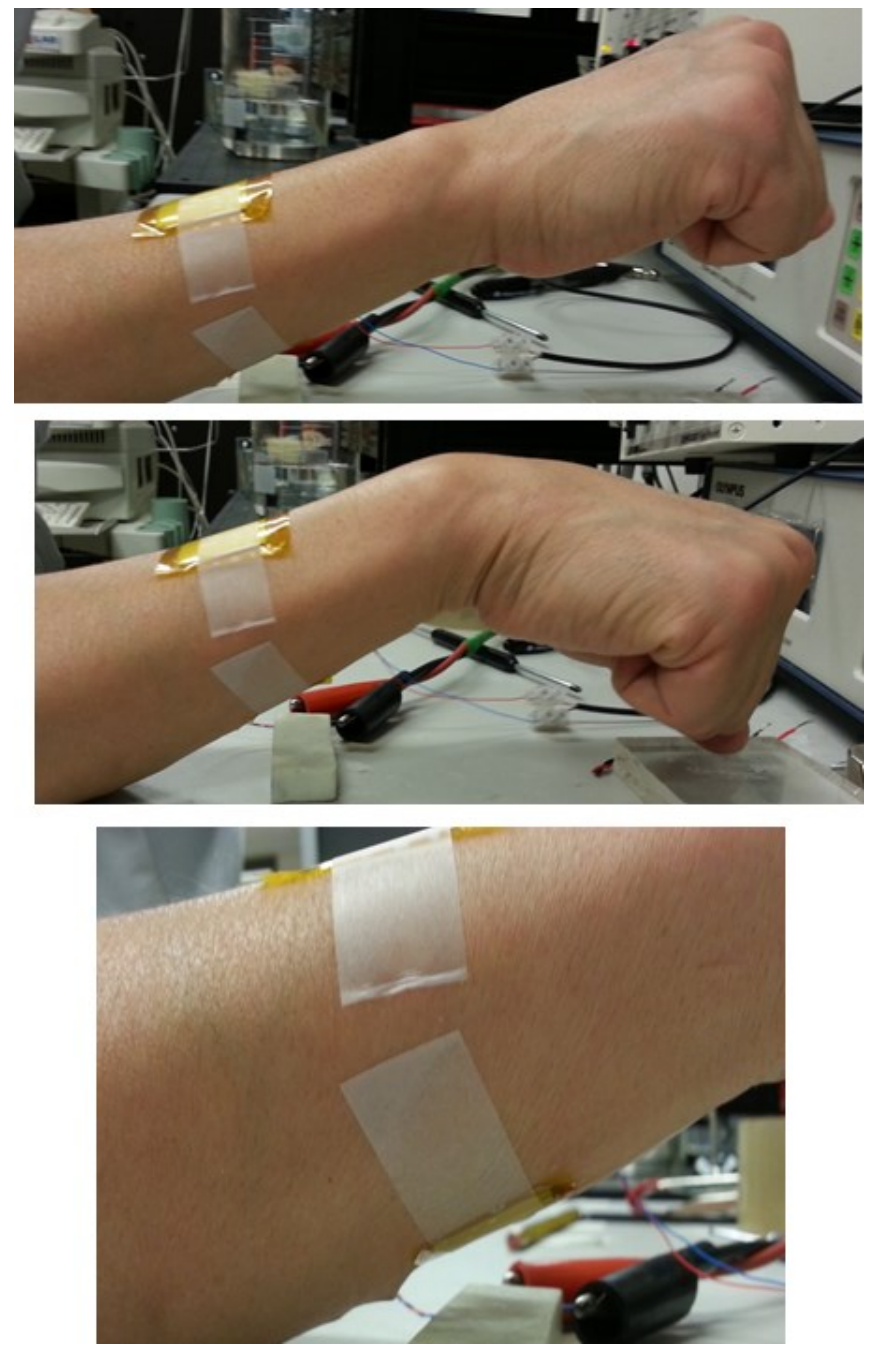

Figure 6.7: Wearable ultrasonic sensor attached onto forearm to monitor isotonic muscle contraction by through-transmission technique. 


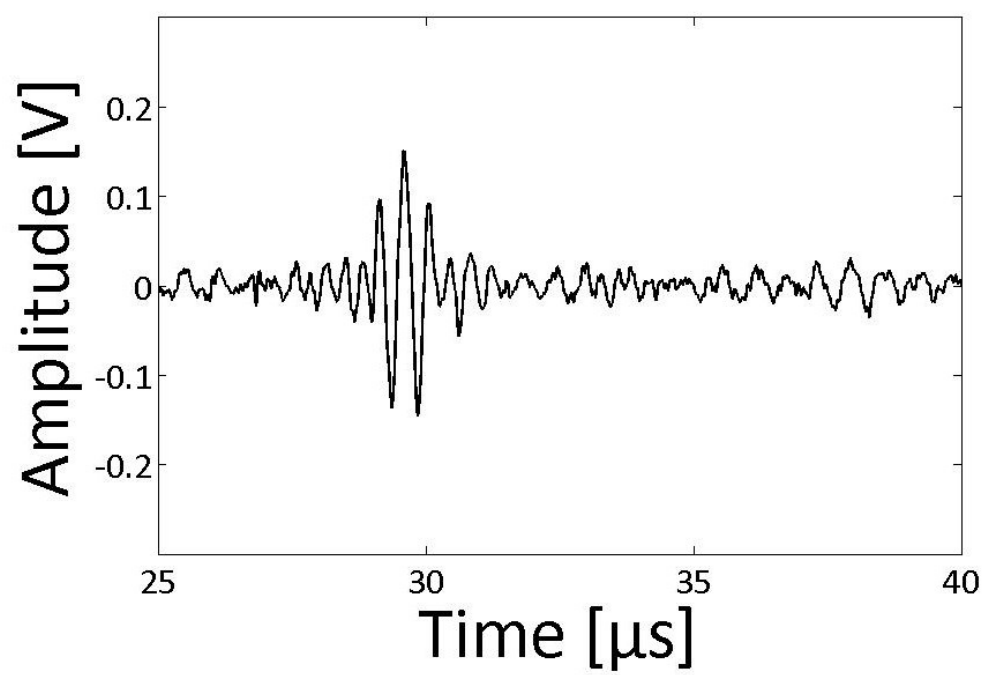

Figure 6.8: The received ultrasonic signal passed through the forearm tissue by transmission-through technique.

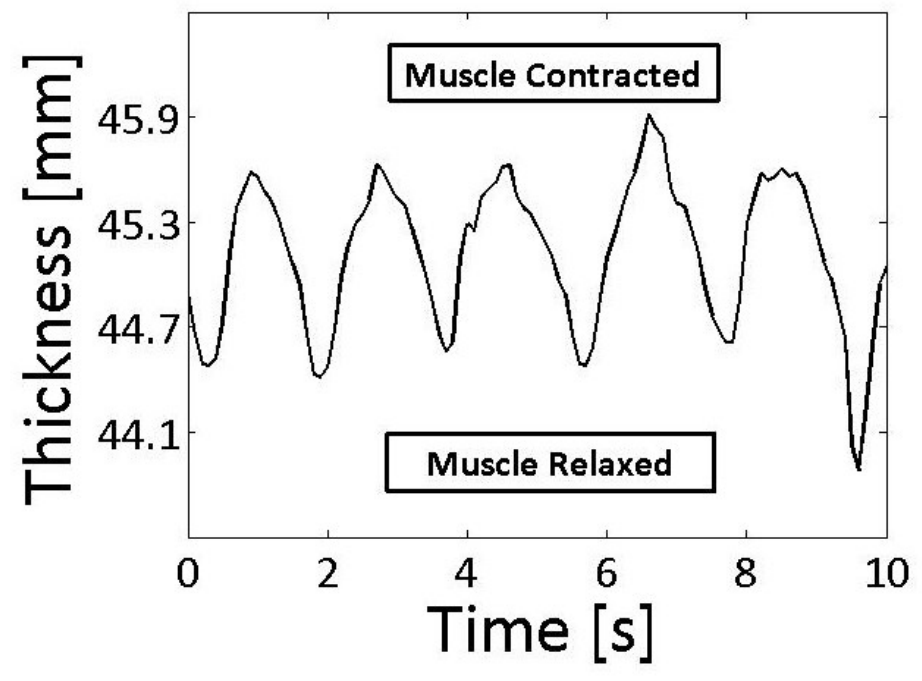

Figure 6.9: Thickness variation during forearm muscle contraction monitored by transmission-through technique. 


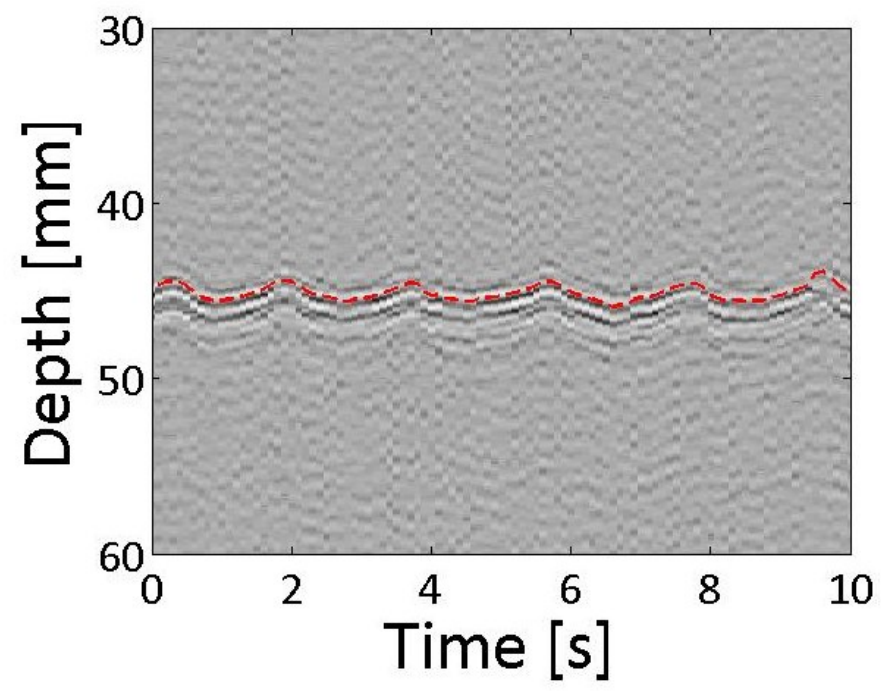

Figure 6.10: M-mode image of ultrasound measurement of forearm tissues by transmission-through technique.

\subsection{Contraction of Finger Muscle}

The purpose of this experiment is to demonstrate the feasibility of monitoring the thickness change of a small and thin muscle such as the finger muscle using the developed sensor. Also, it shows the flexibility of the developed sensor, giving that the ability to fit onto a small body surface.

An index finger was chosen to measure the change in thickness during an isometric contraction (static) using the pulse-echo technique. A $10 \mathrm{~mm} \times 10 \mathrm{~mm}$ developed sensor was attached above the proximal phalange bone of the index finger of the subject's left hand, while the hand was placed on a table as shown in Figure 6.11. During the measurement, the index finger was voluntarily applying force against the table without moving the finger, allowing an isometric contraction of the finger muscle. The ultrasonic signals reflected from the interface between the muscle and the bone, were 
clearly observed at a time of $3.5 \mu \mathrm{s}$, as shown in Figure 6.12. The variation of the tissue thickness between the sensor and the bone was measured using the time delay of the reflected signals. The result is presented in Figure 6.13. Also, the M-mode image illustrates the muscle thickness change, as shown in Figure 6.14. The thickness of the tissue at the relaxed state (where there is no force applied) was around $3.02 \mathrm{~mm}$, where it was around $3.39 \mathrm{~mm}$ during the contraction. Thus, the finger muscle displacement during contraction was around $370 \mu \mathrm{m}(11 \%)$, in accordance with the isometric muscle contraction preformed.

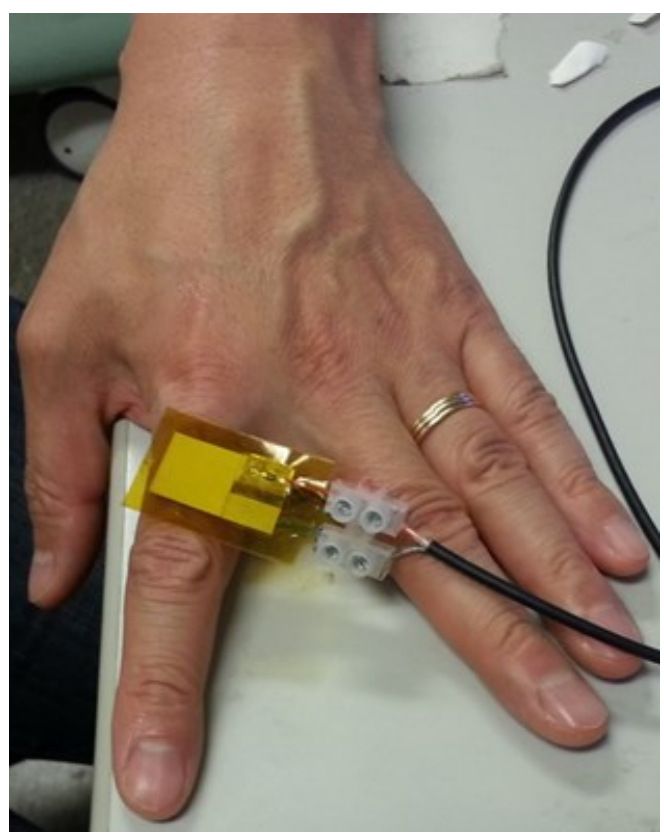

Figure 6.11: Wearable ultrasonic sensor attached into finger to monitor isometric muscle contraction. 


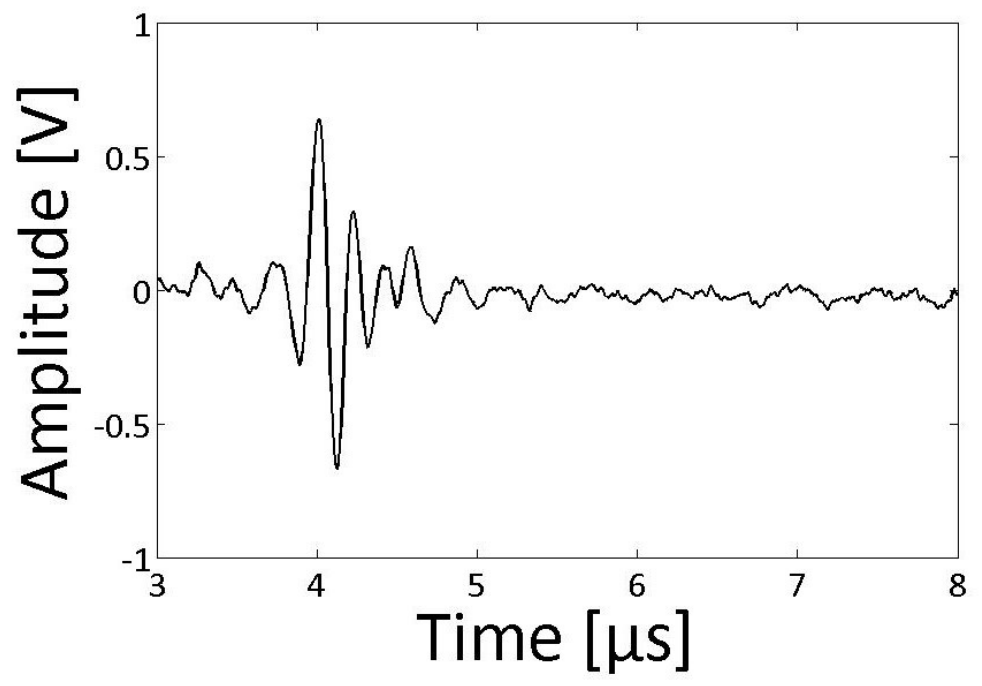

Figure 6.12: Ultrasonic signal reflected from the tissue-bone interface at the index finger.

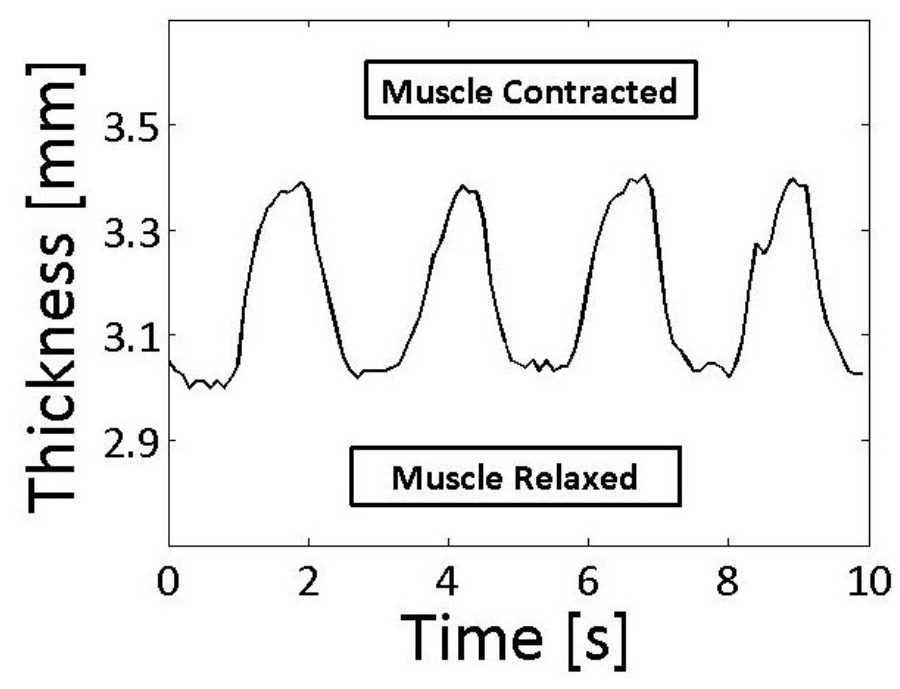

Figure 6.13: Thickness variation during finger muscle isometric contraction. 


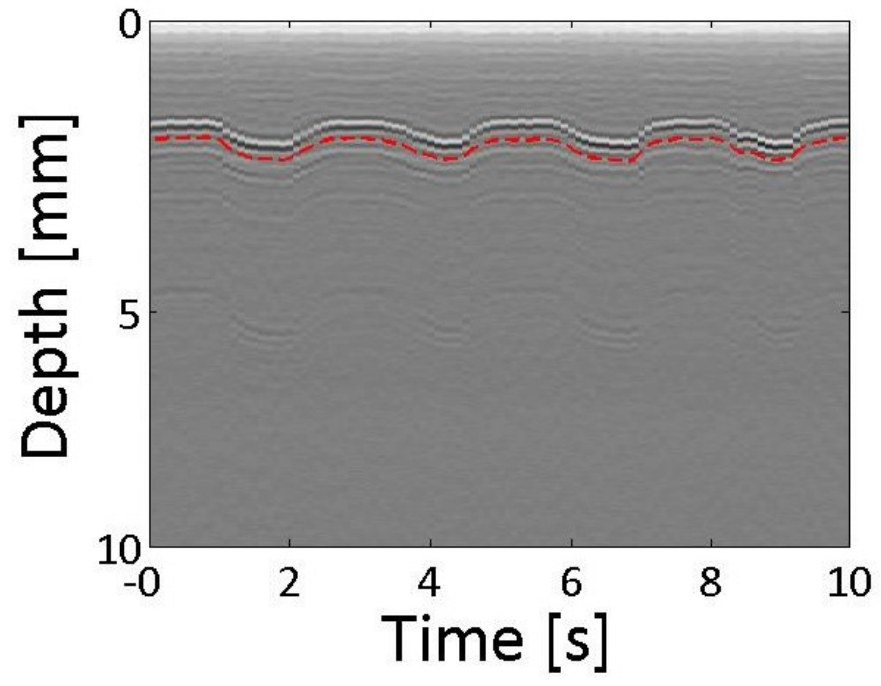

Figure 6.14: M-mode image of ultrasound measurement at finger.

\subsection{Penetration Depth}

In order to evaluate the sensor's efficiency in terms of ultrasonic penetration depth, an in vivo experiment was performed to measure the penetration depth of the ultrasonic wave transmitted by the sensor through a human subject. The developed sensor was attached to the posterior side of a human leg. The active area of the sensor was 15 $\mathrm{mm} \times 15 \mathrm{~mm}\left(=225 \mathrm{~mm}^{2}\right)$. The sensor was driven by an ultrasonic pulser/receiver (5900PR, OLYMPUS) and the received signals were acquired by a PCI digitizer (ATS 460, AlazarTech). Figure 6.15 shows the reflected ultrasonic signal at the tibia bone. The reflected signal was clearly observed at the depth of $23.5 \mathrm{~mm}$. This demonstrates that using the pulse-echo technique, the sensor is capable of monitoring muscle contractions at a depth of at least $23.5 \mathrm{~mm}$. 


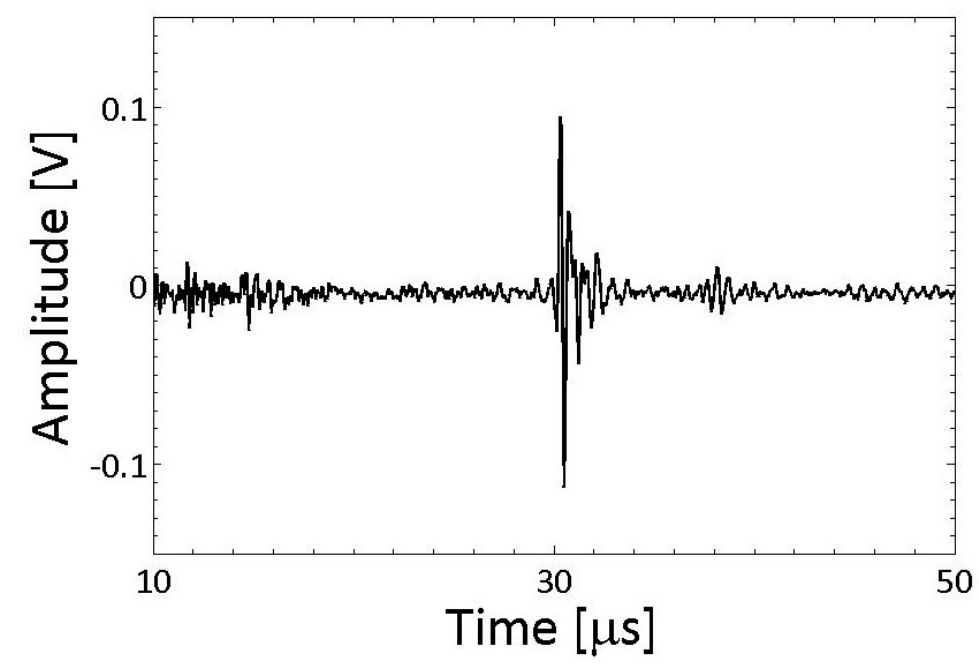

Figure 6.15: Ultrasonic signal reflected from muscle-tibia bone interface at leg. 


\section{Chapter 7: Conclusions and Future Works}

The following chapter concludes the thesis findings and provides suggestions for future work.

\subsection{Conclusions}

This thesis presents the development of a wearable ultrasonic sensor to monitor muscle contractions. The sensor was constructed using a PVDF piezoelectric polymer film, sandwiched by top and bottom electrodes and sealed by protection/insulation layers. The flexibility and lightweight of the sensor allows the developed sensor to be attached to a human body surface, and monitor muscle contractions without restricting its motion. The performance and frequency characteristics of the developed sensor were investigated by numerical simulations and experiments. The numerical simulation model of the sensor is based on Mason's electric equivalent circuit model of piezoelectric resonators. Also, the internal losses of piezoelectric polymers were considered in the mathematical representation of the numerical simulation model for an accurate prediction of its ultrasonic performance.

The numerical simulation and experimental results showed that the developed sensor operated in dual frequencies. This dual frequencies behavior is due to the effect of the non-piezoelectric layers, specifically of the silicone adhesive layers. The frequency response of the developed sensor was improved to a single operating frequency by adding 
a silicon backing material to the sensor design. Also, the investigation of the ultrasonic signal strength of the sensor showed that in the experimental configuration employed in this thesis, the larger the sensor area provided the greater the signal strength.

Further experiments were conducted to demonstrate the capability of developed wearable sensor for monitoring static and dynamic muscle contractions. The tissue thickness variation due to muscle contraction was successfully obtained using the developed ultrasonic sensor.

\subsection{Future Works}

As stated in the objective of this thesis research, the major purpose of the work was to develop a wearable ultrasonic sensor for muscle monitoring. The research done was a first step in the direction of perfecting a wearable ultrasonic system able to monitor muscle activities continuously and in real-time. Thus, there are more work to be done in the future. The following section outlines some suggestions for future research.

The developed sensor has room for improvement. A suggestion would be the enhancement of the ultrasonic signal strength and the signal-to-noise ratio. One way to do this could be to use a multilayer PVDF film in the design of the sensor. Another improvement could be introduction of a multiple transducers in a geometric array configuration within the sensor which may enhance the alignment of the sensor with the motion of the muscle. Also, some improvement could be done on the lead wired connection in order to reduce the noises and to enhance the reliability

Moreover, the acoustic output of the developed sensor should be evaluated to ensure that the developed sensor is safe for the human body and meets the safety 
standards of Health Canada and USA Food and Drug Administration (FDA) for ultrasonic transducers.

Finally, there is a large amount of work to be done with the ultrasonic system. A wearable and portable ultrasonic system must be developed to achieve a practical means of continuous and real-time muscle monitoring outside clinics and laboratories, such as in homes and outdoor environments. It also requires the development of motion estimation methods using robust digital signal processing techniques.

Besides, it should be noted that the applications of the wearable ultrasonic developed sensor are not limited only to muscle monitor. It can also be used for various physiological activities associated with internal tissue thickness changes and motion, such as heart-beat, heart wall movement, breath, swallowing, and bladder volume. 


\section{References}

[1] B. Juul-Kristensen, F. Bojsen-Møller, E. Holst, and C. Ekdahl, "Comparison of muscle sizes and moment arms of two rotator cuff muscles measured by ultrasonography and magnetic resonance imaging," European journal of ultrasound, vol. 11, pp. 161-173. 2000.

[2] J.-Y. Guo, Y.-P. Zheng, H.-B. Xie, and X. Chen, "Continuous monitoring of electromyography (EMG), mechanomyography (MMG), sonomyography (SMG) and torque output during ramp and step isometric contractions," Medical engineering \& physics, vol. 32, pp. 1032-1042, 2010.

[3] K. Masuda, T. Masuda, T. Sadoyama, M. Inaki, and S. Katsuta, "Changes in surface EMG parameters during static and dynamic fatiguing contractions," Journal of Electromyography and Kinesiology, vol. 9, pp. 39-46, 1999.

[4] S. Pillen, I. M. P. Arts, and M. J. Zwarts, "Muscle ultrasound in neuromuscular disorders," Muscle \& nerve, vol. 37, pp. 679-693, 2008.

[5] L. Sherwood, Human physiology: from cells to systems, 7th ed. Brooks/Cole, 2010.

[6] E. N. Marieb and K. Hoehn, Human anatomy and physiology, 7th ed. Pearson, 2007.

[7] P. Klavora, Foundations of Kinesiology: Studying Human Movement and Health, 2nd ed. Sport Books, 2010.

[8] P. K. Kasi, L. S. Krivickas, M. Meister, E. Chew, P. Bonato, M. Schmid, G. Kamen, P. Liu, and E. A. Clancy, "Characterization of motor unit behavior in patients with amyotrophic lateral sclerosis," in Neural Engineering, 4th International IEEE/EMBS, 2009, pp. 10-13.

[9] A. L. Hof, "EMG and muscle force: An introduction," Human Movement Science, vol. 3, pp. 119-153, 1984.

[10] M. A. Mañanas, R. Jané, J. A. Fiz, J. Morera, and P. Caminal, "Study of myographic signals from sternomastoid muscle in patients with chronic obstructive pulmonary disease," IEEE Transactions on Biomedical Engineering, vol. 47, pp. 674-681, 2000.

[11] J.-Y. Hogrel, "Clinical applications of surface electromyography in neuromuscular disorders.," Clinical neurophysiology, vol. 35, pp. 59-71, 2005. 
[12] A. F. Mannion and P. Dolan, "The effects of muscle length and force output on the EMG power spectrum of the erector spinae," Journal of Electromyography and Kinesiology, vol. 6, pp. 159-168, 1996.

[13] M. Pozzo, E. Merlo, D. Farina, G. Antonutto, R. Merletti, and P. E. Di Prampero, "Muscle-fiber conduction velocity estimated from surface emg signals during explosive dynamic contractions," Muscle \& nerve, vol. 29, pp. 823-833, 2004.

[14] D. Farina and R. Merletti, "A novel approach for estimating muscle fiber conduction velocity by spatial and temporal filtering of surface EMG signals," IEEE Transactions on Biomedical Engineering, vol. 50, pp. 1340-1351, 2003.

[15] M. Tanaka, T. Okuyama, and K. Saito, "Study on evaluation of muscle conditions using a mechanomyogram sensor," in IEEE International Conference on Systems, Man, and Cybernetics, 2011, pp. 741-745.

[16] K. Akataki, K. Mita, and Y. Itoh, "Relationship between mechanomyogram and force during voluntary contractions reinvestigated using spectral decomposition," European journal of applied physiology and occupational physiology, vol. 80, pp. 173-179, 1999.

[17] G. OLaighin, B. J. Broderick, M. Clarke-Moloney, F. Wallis, and P. A. Grace, “A technique for the computation of lower leg muscle volume from MRI images in the context of venous return," in International Conference of the IEEE Engineering in Medicine and Biology Society, 2007, 2007, vol. 2007, pp. 951-954.

[18] A. F. Mannion, N. Pulkovski, D. Gubler, M. Gorelick, D. O'Riordan, T. Loupas, P. Schenk, H. Gerber, and H. Sprott, "Muscle thickness changes during abdominal hollowing: an assessment of between-day measurement error in controls and patients with chronic low back pain," European spine journal, vol. 17, pp. 494$501,2008$.

[19] G. Misuri, S. Colagrande, M. Gorini, I. Iandelli, M. Mancini, R. Duranti, and G. Scano, "In vivo ultrasound assessment of respiratory function of abdominal muscles in normal subjects," European Respiratory Journal, vol. 10, pp. 28612867, 1997.

[20] P. W. Hodges, L. H. M. Pengel, R. D. Herbert, and S. C. Gandevia, "Measurement of muscle contraction with ultrasound imaging," Muscle \& nerve, vol. 27, pp. 682692, 2003.

[21] M. Noorkoiv, K. Nosaka, and A. J. Blazevich, "Assessment of quadriceps muscle cross-sectional area by ultrasound extended-field-of-view imaging," European journal of applied physiology, vol. 109, pp. 631-639, 2010. 
[22] D. Ensminger and L. J. Bond, Ultrasonics: Fundamentals, Technologies, and Applications, 3rd ed. CRC Press, 2012.

[23] J. L. Prince and J. M. Links, Medical Imaging Signal and Systems. Pearson, 2006.

[24] D. A. Christensen, Ultrasonic Bioinstrumentation. John Wiley \& Sons, 1988.

[25] W. R. Hedrick, D. L. Hykes, and D. E. Starchman, Ultrasound Physics and Instrumentation, 4th ed. Elsevier Mosby, 2005.

[26] H. Kuttruff, Ultrasonics Fundamentals and Applications. Elsevier Science, 1991.

[27] V. Gibbs, D. Cole, and A. Sassano, Ultrasound Physics and Technology: How, Why, and when. Elsevier sciences, 2011.

[28] P. Hoskins, A. Thursh, K. Martin, and T. Whittingam, Diagnostic Ultrasound: Physics and Equipment, 1st ed. Greenwich Medical Media, 2003.

[29] F. W. Kremkau, Diagnostic Ultrasound: Principles and Instruments, 7th ed. Elsevier, 2006.

[30] W. D. O'Brien Jr, "Assessing the risks for modern diagnostic ultrasound imaging," Japanese journal of applied physics, vol. 37, pp. 2781-2788, 1998.

[31] J. Woo, "A short history of the development of ultrasound in obstetrics and gynecology," 2002. [Online]. Available: http://www.obultrasound.net/history2.html. [Accessed: 20-Aug-2013].

[32] FUJIFILM SonoSite, "NanoMaxx Ultrasound Machine." [Online]. Available: http://www.sonosite.ca/ultrasound-products/nanomaxx. [Accessed: 20-Aug-2013].

[33] GE_Healthcare, "Vscan Pocket Ultrasound." [Online]. Available: https://vscan.gehealthcare.com/en-ca/gallery/a-quick-look-at-vscan-canada. [Accessed: 20-Aug-2013].

[34] Siemens, "ACUSON P10 ultrasound system." [Online]. Available: http://www.healthcare.siemens.com/ultrasound/cardiovascular/acuson-p10ultrasound-system. [Accessed: 20-Aug-2013].

[35] Current Solutions, "US Pro $2000^{\mathrm{TM}}$, Professional Series." [Online]. Available: http://www.currentsolutionsnow.com/ultrasound.html. [Accessed: 20-Aug-2013].

[36] Interson Corporation, "SeeMore ${ }^{\mathrm{TM}}$ Ultrasound Imaging USB Probes." [Online]. Available: http://www.interson.com/products/seemore-153-usb-probes. 
[37] P. Bonato, "Wearable sensors/systems and their impact on biomedical engineering," IEEE Engineering in Medicine and Biology Magazine, vol. 22, pp. 18-20, 2003.

[38] A. Lanata, E. P. Scilingo, and D. De Rossi, "A multimodal transducer for cardiopulmonary activity monitoring in emergency," IEEE Transactions on Information Technology in Biomedicine, vol. 14, pp. 817-825, 2010.

[39] A. Lanata, E. P. Scilingo, R. Francesconi, G. Varone, and D. De Rossi, "New ultrasound-based wearable system for cardiac monitoring," in IEEE Conference on Sensors, 2006, pp. $489-492$.

[40] A. J. Zuckerwar, R. A. Pretlow, J. W. Stoughton, and D. A. Baker, "Development of a piezopolymer pressure sensor for a portable fetal heart rate monitor.," IEEE transactions on bio-medical engineering, vol. 40, pp. 963-969, 1993.

[41] M. Roham, E. Saldivar, S. Raghavan, M. Zurcher, J. Mack, and M. Mehregany, “A mobile wearable wireless fetal heart monitoring system," in International Symposium on Medical Information \& Communication Technology (ISMICT), 2011, pp. 135-138.

[42] C.-C. Huang, P.-Y. Lee, P.-Y. Chen, and T.-Y. Liu, "Design and implementation of a smartphone-based portable ultrasound pulsed-wave Doppler device for blood flow measurement," IEEE transactions on ultrasonics, ferroelectrics, and frequency control, vol. 59, pp. 182-188, 2012.

[43] M. T. Rise, W. E. Bradley, and D. A. Frohrib, "An ultrasonic bladder-volume sensor," IEEE Transactions on Biomedical Engineering, vol. BME-26, pp. 709$711,1979$.

[44] M. N. K. Kristiansen, J. C. Djurhuus, and H. Nygaard, "Design and evaluation of an ultrasound-based bladder volume monitor," Medical and Biological Engineering and Computing, vol. 42, pp. 762-769,. 2004.

[45] A. Bhuyan, J. W. Choe, B. C. Lee, P. Cristman, Ö. Oralkan, and B. T. KhuriYakub, "Miniaturized, wearable, Ultrasound Probe for On-Demand Ultrasound Screening," in IEEE International, Ultrasonics Symposium, 2011, pp. 1060 - 1063.

[46] G. K. Lewis Jr, M. D. Langer, C. R. Henderson Jr, and R. Ortiz, "Design and evaluation of a wearable self-applied therapeutic ultrasound device for chronic myofascial pain," Ultrasound in medicine \& biology, vol. 39, pp. 1429-1439, 2013.

[47] Esaote, "LA40 ultrasonic probe." [Online]. Available: http://www.esaote.com/modules/core/page.asp?p=US_TRANSDUCERS. [Accessed: 27-Aug-2013]. 
[48] OLYMPUS, "Contact Transducers." [Online]. Available: http://www.olympusims.com/en/ultrasonic-transducers/contact-transducers/. [Accessed: 24-Aug-2013].

[49] Health Canada, "Device Licence Applications for Ultrasound Diagnostic Systems and Transducers," Health Canada, Heatlh Products and Foods Branch, 2006.

[50] Food and Drug Administration, "Information for Manufacturers Seeking Marketing Clearance of Diagnostic Ultrasound Systems and Transducers," U.S. Department of Health and Human Services, Food and Drug Administration, Center for Devices and Radiological Health, 2008.

[51] W. A. Smith, "Composite piezoelectric materials for medical ultrasonic imaging transducers--a review," IEEE Ultrasonics Symposium, pp. 249-256, 1986.

[52] J. A. Gallego-Juarez, "Piezoelectric ceramics and ultrasonic transducers," Journal of Physics E: Scientific Instruments, vol. 22, pp. 804-816, 1989.

[53] J. D. N. Cheeke, Fundamentals and Applications of Ultrasonic Waves. CRC press, 2002.

[54] G. D. Ludwig, "The velocity of sound through tissues and the acoustic impedance of tissues," The Journal of the Acoustical Society of America, vol. 22, pp. 862-866, 1950.

[55] L. F. Brown, "Design considerations for piezoelectric polymer ultrasound transducers," IEEE transactions on ultrasonics, ferroelectrics, and frequency control, vol. 47, pp. 1377-1396, 2000.

[56] W. P. Mason, Electromechanical Transducer and Wave Filters, 2nd ed. Van Nostrand Reinhold, 1948.

[57] H. Ohigashi, "Ultrasonic transducers in the megahertz range," in The Application of Ferroelectric Polymers, T. T. Wang, J. M. Herbert, and A. M. Glass, Eds. Routledge, Chapman, and Hall, 1988, pp. 237-273.

[58] E. K. Sittig, "Transmission parameters of thickness-driven piezoelectric transducers arranged in multilayer configurations," IEEE transactions on Sonics and Ultrasonics, vol. 14, pp. 167-174, 1967.

[59] H. Ohigashi, T. Itoh, K. Kimura, T. Nakanishi, and M. Suzuki, "Analysis of frequency response characteristics of polymer ultrasonic transducers," Japanese journal of applied physics, vol. 27, pp. 354-360, 1988.

[60] G. S. Kino, Acoustic Waves: Devices, Imaging, and Analog Signal Processing. Prentice-Hall, 2000. 
[61] O. G. Molina, "Ultrasonic couplant gel compositions and method for employing same," 1982.

[62] H. Azhari, Basics of Biomedical Ultrasound for Engineers, 1st ed. Wiley-IEEE Press, 2010.

[63] Alazartech, "ATS460 PCI Digitizer.” [Online]. Available: http://www.alazartech.com/products/ATS460_v_1_2F.pdf. [Accessed: 18-Jul2013].

[64] A. R. Selfridge, "Approximate material properties in isotropic materials," IEEE transactions on sonics and ultrasonics, vol. 32, pp. 381-394, 1985.

[65] C.-K. Jen, D. R. Franca, Z. Sun, and I. Ihara, "Clad polymer buffer rods for polymer process monitoring," Ultrasonics, vol. 39, pp. 81-89, 2001. 